\title{
Autenticação e comunicação segura em dispositivos móveis de poder computacional restrito
}

\author{
Rafael Will Macedo de Araujo
}

\author{
DisSERTAÇÃO APRESENTADA \\ $\mathrm{AO}$ \\ Instituto DE MATEMÁticA E EstatísticA \\ DA \\ Universidade DE SÃo PaUlo \\ PARA \\ OBTENÇÃO DO TÍTULO \\ $\mathrm{DE}$ \\ Mestre em CiÊnCIAS
}

Programa: Mestrado em Ciência da Computação

Orientador: Prof. Dr. Routo Terada

Durante o desenvolvimento deste trabalho

o autor recebeu auxílio financeiro do $\mathrm{CNPq}$

São Paulo, outubro de 2013 


\section{Autenticação e comunicação segura em dispositivos móveis de poder computacional restrito}

Esta dissertação contém as correções e alterações sugeridas pela Comissão Julgadora durante a defesa realizada por Rafael Will Macedo de Araujo em 31/10/2013.

O original encontra-se disponível no Instituto de

Matemática e Estatística da Universidade de São Paulo.

Comissão Julgadora:

- Prof. Dr. Routo Terada (orientador) - IME-USP

- Prof. Dr. Roberto Hirata Junior - IME-USP

- Profa. Dra. Cíntia Borges Margi - EP-USP 


\section{Agradecimentos}

A Deus, pela saúde e sabedoria que permitiram a conclusão deste trabalho.

Aos meus pais Naum e Wâner, pelo suporte contínuo, por sempre acreditarem em mim e pela dedicação que têm me dado em todos os momentos de minha vida, e a todos os familiares que me apoiaram.

À minha namorada Lilian, por seu amor, afeto e palavras de carinho que foram fundamentais em todos os momentos do mestrado, e aos seus pais Valdson e Lenilde, pelo apoio e palavras de motivação.

Ao meu orientador e professor Routo Terada, pela confiança e por guiar-me durante o desenvolvimento desta dissertação, com sua paciência, experiência e conhecimento.

Aos membros da banca de qualificação e da comissão julgadora (professores Marco Aurélio Gerosa, Cíntia Borges Margi, Roberto Hirata Junior), pelas valiosas sugestões, questionamentos e comentários.

Aos amigos e membros do Laboratório de Segurança de Dados (LSD) do IME-USP, pelas discussões e companheirismo. Em especial, a Denise Goya pela parceria e conhecimentos compartilhados.

Aos demais amigos da USP que estiveram comigo desde a época das disciplinas e grupos de estudo.

Aos amigos de longa data de Aracaju, que apesar da distância continuaram me apoiando e torcendo pelo meu sucesso.

A todos os professores e funcionários do IME-USP, que colaboraram com minha formação e viabilizaram a produção desta obra.

Ao Conselho Nacional de Desenvolvimento Científico e Tecnológico (CNPq), pelo apoio financeiro.

A todos, meus sinceros agradecimentos! 


\section{Resumo}

Protocolos de autenticação e de estabelecimento de chaves são peças fundamentais em implementações de segurança para comunicação de dispositivos eletrônicos. Em aplicações que envolvam dispositivos com poder computacional restrito (tais como smartphones ou tablets) comunicando-se com um servidor, é primordial a escolha de protocolos eficientes e que necessitem de uma infraestrutura mais simples. Neste trabalho selecionamos e implementamos protocolos de acordo de chave seguros nos modelos de criptografia de chave pública baseado em identidade (ID-based) e sem certificado (Certificateless) em plataformas com processadores ARM. Comparamos tempos de execução, utilização de memória e uso do canal de comunicação.

Palavras-chave: protocolo de acordo de chave, criptografia baseada em identidade, criptografia sem certificado, emparelhamento bilinear, criptografia sobre curva elíptica, dispositivos móveis. 


\section{Abstract}

Protocols for authentication and key establishment are fundamental parts in security implementations for electronic devices communication. In applications involving devices with limited computational power (such as smartphones and tablets) communicating with a server, the choice of efficient protocols that require a simpler infrastructure is essential. In this work we select and implement secure key agreement protocols in ID-based and Certificateless public key cryptography models on ARM processor platforms. We also compare running times, memory and network usage.

Keywords: key agreement protocol, ID-based cryptography, certificateless cryptography, bilinear pairing, elliptic curve cryptography, mobile devices. 


\section{Sumário}

$\begin{array}{ll}\text { Lista de Abreviaturas } & \text { ix }\end{array}$

Lista de Figuras

Lista de Tabelas $\quad$ xiii

1 Introdução $\quad 1$

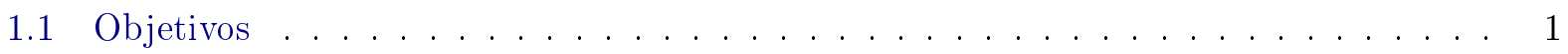

1.2 Organização do trabalho . . . . . . . . . . . . . . . . . 1

2 Fundamentos de Criptografia $\quad 3$

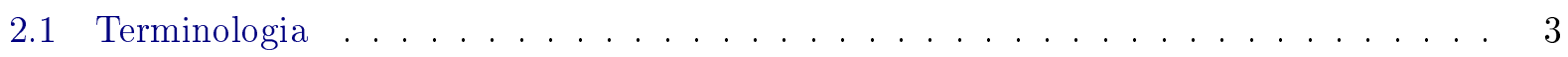

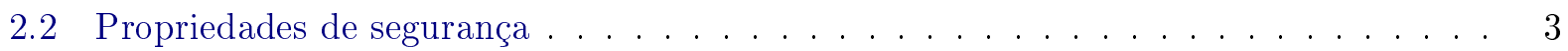

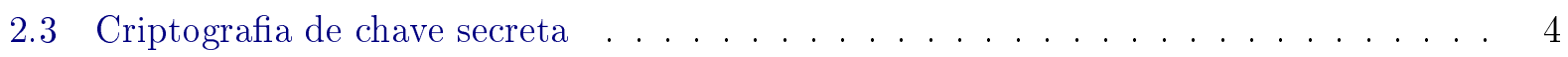

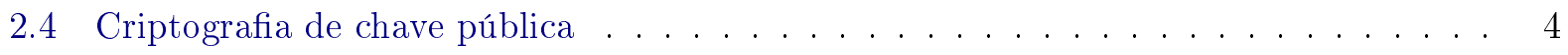

2.4 .1 Assinatura digital . . . . . . . . . . . . . . . . . 5

2.4 .2 Infraestrutura de chaves públicas . . . . . . . . . . . . . . 6

3 Emparelhamentos Bilineares $\quad 9$

3.1 Complexidade de algoritmos e problemas computacionais de interesse . . . . . . . . . 10

3.1.1 Problema do logaritmo discreto . . . . . . . . . . . . . . . . . 11

3.1.2 Problema do logaritmo discreto sobre curvas elípticas . . . . . . . . . . . . 11

3.1.3 Outros problemas relacionados . . . . . . . . . . . . . . . . 11

4 Modelos Alternativos de Criptografia de Chave Pública $\quad 13$

4.1 Modelo de chave pública baseado em identidade . . . . . . . . . . . . . . . . . 13

4.2 Modelo de chave pública sem certificado . . . . . . . . . . . . . . . . . . . 14

4.3 Comprimento das chaves . . . . . . . . . . . . . . . . . . . . 16

4.4 Protocolos de acordo de chave . . . . . . . . . . . . . . . . . . 16

4.4 Atributos de segurança . . . . . . . . . . . . . . . . . . 17

4.4 .2 Modelos de segurança . . . . . . . . . . . . . . . . . 18

5 Protocolos Estudados $\quad 21$

5.1 Escolha dos protocolos . . . . . . . . . . . . . . . . . . . 21

5.2 Protocolos no modelo baseado em identidade . . . . . . . . . . . . . . . . 21

5.2 .1 Protocolo Huang-Cao . . . . . . . . . . . . . . . . . . . . 23 
5.2 .2 Protocolo Hu-Liu-Zhang . . . . . . . . . . . . . . . . . . . . . . . 23

5.2 .3 Protocolo Chow-Choo . . . . . . . . . . . . . . . . . . 24

5.2 .4 Protocolo Ni-Chen-Li-Hao . . . . . . . . . . . . . . . . . 25

5.2 .5 Protocolo Ni-Chen-Li . . . . . . . . . . . . . . . 26

5.3 Protocolos no modelo sem certificado . . . . . . . . . . . . . . . . 27

5.3 .1 Protocolo Lippold-Boyd-González Nieto . . . . . . . . . . . . . . . . 28

5.3 .2 Protocolo Goya-Okida-Terada . . . . . . . . . . . . . . . . . . 29

5.3 .3 Protocolo Goya-Nakamura-Terada-3 . . . . . . . . . . . . . . . . 31

5.3 .4 Protocolo Goya-Nakamura-Terada-4 . . . . . . . . . . . . . . . . . 31

5.3.5 Protocolo Goya-Nakamura-Terada-1 . . . . . . . . . . . . . . . . . . . . . . . . . . .

5.3 .6 Protocolo Goya-Nakamura-Terada-2 . . . . . . . . . . . . . . . . 33

5.4 Adaptação para emparelhamento assimétrico . . . . . . . . . . . . . . 34

6 Experimentos $\quad 35$

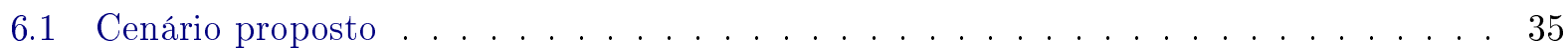

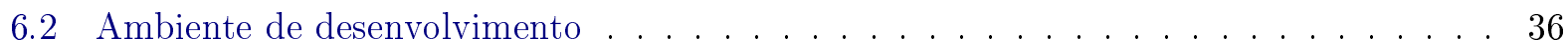

6.3 Curvas elípticas e custo das operações . . . . . . . . . . . . . . . . . . 37

6.4 Adaptação eficiente para emparelhamento assimétrico . . . . . . . . . . . . . . . . 40

6.5 Comparação de desempenho entre os protocolos analisados . . . . . . . . . . . . . . . 41

6.6 Considerações finais sobre os resultados . . . . . . . . . . . . . . . . 50

7 Conclusões $\quad \mathbf{5 3}$

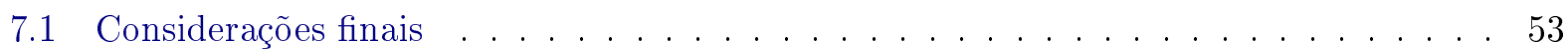

7.2 Sugestões para pesquisas futuras $\ldots \ldots \ldots \ldots \ldots \ldots \ldots \ldots$

A Fundamentos Matemáticos $\quad 55$

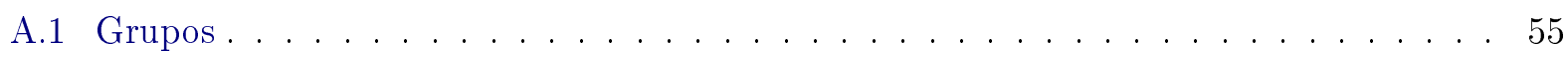

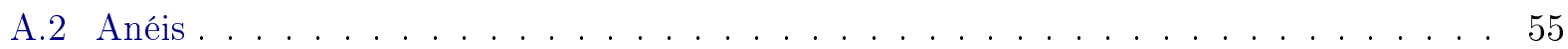

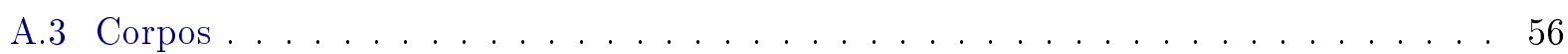

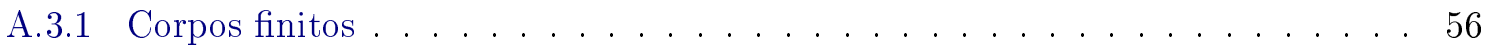

A.4 Curvas elípticas . . . . . . . . . . . . . . . . . . 56

A.4.1 Ordem de curvas elípticas . . . . . . . . . . . . . . . 57

A.4.2 Lei de grupos em curvas elípticas . . . . . . . . . . . . . . . . . . 57

B Intervalos de Confiança dos Experimentos $\quad 59$

$\begin{array}{ll}\text { Referências Bibliográficas } & 63\end{array}$ 


\title{
Lista de Abreviaturas
}

\author{
AES Advanced Encription Standard (algoritmo utilizado para criptografia simétrica) \\ DES Data Encription Standard (algoritmo utilizado para criptografia simétrica) \\ DLP Problema do logaritmo discreto (Discrete Logarithm Problem) \\ ECC Criptossistema de Curvas Elípticas (Elliptic Curve Cryptosystem) \\ IBE Encriptação baseada em identidades (Identity-Based Encryption) \\ ICP Infraestrutura de chaves públicas \\ KGC Centro gerador de chaves (Key Generation Center) \\ JNI Java Native Interface (permite que a máquina virtual Java acesse código nativo) \\ PKI Infraestrutura de chaves públicas (Public Key Infrastructure) \\ PKG Gerador de chaves privadas (Private Key Generator) \\ RSA Acrônimo de Rivest, Shamir, Adleman (algoritmo utilizado para criptografia de chave pública)
}




\section{Lista de Figuras}

2.1 Exemplo de certificado digital. . . . . . . . . . . . . . . . . . . . 7

2.2 Modelo de certificação em árvore . . . . . . . . . . . . . . . . . . 7

2.3 Modelo de certificação em floresta. . . . . . . . . . . . . . . . . . . . 8

3.1 Ilustração de um emparelhamento Bilinear (Cesena, 2010) . . . . . . . . . . . . . . 10

4.1 Distribução de chaves na criptografia de chave pública baseada em identidade. . . . . 14

4.2 Distribução de chaves na criptografia de chave pública sem certificado. . . . . . . . 15

4.3 Protocolo de acordo de chave Diffie-Hellman. . . . . . . . . . . . . . . . . . . 17

4.4 Hierarquia dos modelos de segurança para protocolos no modelo sem certificado (Goya, 2011) . . . . . . . . . . . . . . . . . . . . . . 19

5.1 Representação genérica de um protocolo de acordo de chave no modelo baseado em identidades . . . . . . . . . . . . . . . . . . . . 22

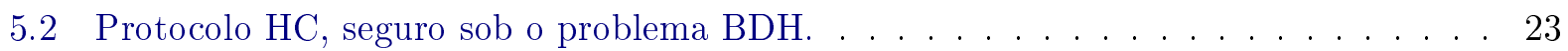

5.3 Protocolo HLZ, seguro sob o problema Gap-BDH. . . . . . . . . . . . . . . . . . 24

5.4 Protocolo CC, seguro sob o problema BDH. . . . . . . . . . . . . . . . . 24

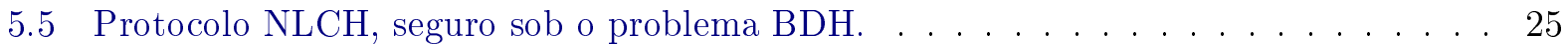

5.6 Protocolo NLCH, seguro sob o problema Gap-BDH. . . . . . . . . . . . . . . 25

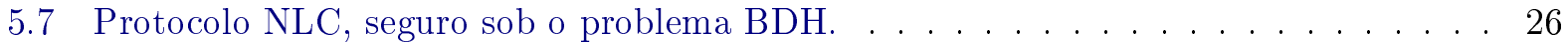

5.8 Representação genérica de um protocolo de acordo de chaves no modelo sem certificado 27

5.9 Protocolo LBG, seguro sob o problema BDH. . . . . . . . . . . . . . 28

5.10 Protocolo LBG, seguro sob o problema Gap-BDH. . . . . . . . . . . . . . . . . 29

5.11 Protocolo GOT, seguro sob o problema BDH. . . . . . . . . . . . . . . 30

5.12 Protocolo GOT, seguro sob o problema Gap-BDH. . . . . . . . . . . . . . . . 30

5.13 Protocolo GNT3, seguro sob o problema BDH. . . . . . . . . . . . . . . . 31

5.14 Protocolo GNT4, seguro sob o problema BDH. . . . . . . . . . . . . . . . . 32

5.15 Protocolo GNT1, seguro sob o problema Gap-BDH. . . . . . . . . . . . . . . . . 33

5.16 Protocolo GNT2, seguro sob o problema Gap-BDH. . . . . . . . . . . . . . . . . 33

6.1 Cenário dos experimentos e comunicação entre os dispositivos: a) Raspberry Pi, b) Motorola Milestone 1, c) Google Nexus 7. . . . . . . . . . . . . . . 36

6.2 Nível de segurança das curvas elípticas escolhidas. . . . . . . . . . . . . . . . . 37

6.3 Comparação entre os tempos de cada operação em curvas binárias (em milissegundos). 39

6.4 Comparação entre os tempos de cada operação em curvas primas (em milissegundos). 40

6.5 Protocolo Huang-Cao assimétrico - caso $1 \ldots \ldots$. . . . . . . . . . . . 41 
6.6 Protocolo Huang-Cao assimétrico - caso $2 \ldots \ldots \ldots \ldots$. . . . . . . . . 41

6.7 Comparação entre os protocolos baseados em identidade, em diferentes níveis de segurança, para o caso sem pré-computação. . . . . . . . . . . . . . . 45

6.8 Comparação entre os protocolos sem certificado, em diferentes níveis de segurança, para o caso sem pré-computação. . . . . . . . . . . . . . . . . . 46

6.9 Comparação entre os protocolos baseados em identidade, em diferentes níveis de segurança, para o caso com pré-computação. . . . . . . . . . . . . . . . 48

6.10 Comparação entre os protocolos sem certificado, em diferentes níveis de segurança, para o caso com pré-computação. . . . . . . . . . . . . . . . . . . 49

A.1 a) soma entre dois pontos $P$ e $Q$ e b) dobramento de um ponto $P \ldots \ldots$. . . . 58 


\section{Lista de Tabelas}

4.1 Comparação entre comprimentos mínimos de chaves (em bits) para diferentes níveis

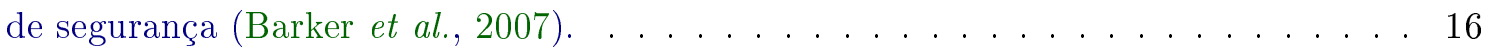

6.1 Tempo de execução das operações (em milissegundos), em diferentes dispositivos e níveis de segurança. . . . . . . . . . . . . . . . . . . 38

6.2 Relação entre protocolos no modelo baseado em identidade e modelos de segurança. . 42

6.3 Relação entre protocolos no modelo sem certificado e modelos de segurança. . . . . . 42

6.4 Quantidade de operações nos protocolos implementados (emparelhamento simétrico). 43

6.5 Quantidade de operações nos protocolos implementados (emparelhamento assimétrico). 43

6.6 Tempos de execução dos protocolos (em milissegundos), sem pré-computação, para diferentes níveis de segurança. . . . . . . . . . . . . . . . . . . . 44

6.7 Tempos de execução dos protocolos (em milissegundos), com pré-computação, para diferentes níveis de segurança. . . . . . . . . . . . . . . . . . . . . . 47

6.8 Uso de memória (em bytes) dos protocolos analisados para diferentes níveis de segu-

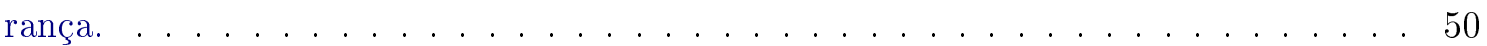

6.9 Comprimento das mensagens trocadas (em bytes) dos protocolos analisados para diferentes níveis de segurança. . . . . . . . . . . . . . . . . . . . 50

B.1 Intervalo de confiança (95\%) dos tempos de execução dos protocolos (em milissegundos), sem pré-computação, para diferentes níveis de segurança. . . . . . . . . . 60

B.2 Intervalo de confiança (95\%) dos tempos de execução dos protocolos (em milissegundos), com pré-computação, para diferentes níveis de segurança. . . . . . . . . . . 61

B.3 Intervalos de confiança (95\%) dos tempos de execução por operação (em milissegundos). 62 


\section{Capítulo 1}

\section{Introdução}

O conceito de criptografia de chave pública surgiu através da busca por soluções para dois problemas intrinsecamente relacionados com o modelo de criptografia simétrica: o de distribuir uma chave secreta e autenticação de usuários, com garantia de irretratabilidade. Ambos os problemas foram tratados por (Diffie e Hellman, 1976), quando nasceu um novo tipo de criptografia: a de chave pública.

Este novo paradigma faz uso de duas chaves relacionadas matematicamente, e por isso é também chamado de criptografia assimétrica. Enquanto uma das chaves deve ser mantida sob sigilo pelo seu proprietário, a outra é divulgada publicamente para que possa ser utilizada por quem deseja se comunicar com usuário dono daquela chave.

No entanto, este novo paradigma trouxe consigo alguns desafios. Um problema central desse modelo é o de legitimação da chave pública. A solução encontrada para este problema foi a certificação das chaves públicas, perante uma autoridade certificadora idônea, através da implantação de uma infra-estrutura de chaves públicas (ICP ou PKI - Public Key Infrastructure). Todavia, esta solução criou uma série de novas dificuldades, como os custos para implantação e manutenção da ICP, maior complexidade para recuperar e validar certificados, além de problemas de revogação dos certificados.

Neste trabalho nos concentramos em dois modelos alternativos de criptografia de chave pública que não fazem uso de certificados digitais: o modelo baseado em identidades, proposto por Shamir (1984) e o modelo sem certificado, proposto por Al-Riyami e Paterson (2003). Dentro dos criptossistemas existentes nesses dois modelos, destacamos uma classe específica, conhecida como protocolos de acordo de chave.

\subsection{Objetivos}

Este trabalho tem como objetivo fazer um levantamento de protocolos de acordo de chave com autenticação, nos modelos de criptografia de chave pública baseado em identidade e sem certificado. Junto a esse levantamento, objetiva-se um mapeamento das propriedades de segurança. Por fim, implementações e medições de desempenho contribuem com um painel comparativo sobre em que contexto de aplicação cada protocolo é mais adequado.

\subsection{Organização do trabalho}

No Capítulo 2 é feita uma revisão dos conceitos básicos de criptografia simétrica e assimétrica, assinatura e certificados digitais. O Capítulo 3 apresenta os fundamentos matemáticos necessários para compreensão dos conceitos que serão expostos no decorrer do texto. No Capítulo 4 temos uma introdução básica aos modelos de criptografia baseado em identidade e sem certificado, suas vantagens e desvantagens, além de uma revisão sobre protocolos de acordo de chave e modelos de segurança. O Capítulo 5 descreve os protocolos selecionados para realização dos experimentos e os 
critérios adotados. No Capítulo 6 apresentamos os experimentos realizados e os resultados obtidos. Finalmente, no Capítulo 7 são feitas considerações finais e conclusões. 


\section{Capítulo 2}

\section{Fundamentos de Criptografia}

Este capítulo apresenta uma visão geral dos conceitos básicos de criptografia, indispensáveis para o melhor entendimento deste trabalho.

\subsection{Terminologia}

No decorrer deste trabalho serão utilizados uma série de termos presentes na literatura especializada em criptografia. Definiremos alguns dos termos mais essenciais para o entendimento deste e dos próximos capítulos a seguir.

- Participante, entidade ou usuário: é alguém ou algo (por exemplo: uma pessoa, um computador, etc) que envia, recebe, ou manipula informação. Quando representarmos duas pessoas que desejam se comunicar, chamaremos essas duas entidades de Alice e Beto, ou simplesmente de entidades $A$ e $B$.

- Adversário, atacante, usuário mal-intencionado, intruso ou espião: é uma entidade que não é nem o remetente nem o destinatário da informação que está sendo transmitida. Seu objetivo é tentar anular o serviço de segurança de informação estabelecido entre duas entidades legítimas que desejam se comunicar. Chamaremos essa entidade mal-intencionada de $\mathcal{C}$ arlos, ou simplesmente $C$.

- Canal (ou linha) de comunicação: meio (por exemplo: linha telefônica, ondas de rádio, Internet, $\mathrm{LAN}^{1}$, etc) por onde trafegam as informações trocadas por participantes de uma comunicação.

- Chave: sequência de símbolos que controla a operação de uma transformação criptográfica.

- Texto legível: mensagem ou informação que uma entidade $A$ (remetente) deseja enviar a uma entidade $B$ (destinatário).

- Texto cifrado ou texto ilegível: resultado de uma transformação criptográfica aplicada ao texto legível, de maneira que não se consiga ter conhecimento da informação sem o conhecimento da chave adequada.

\subsection{Propriedades de segurança}

Para que seja possível estabelecer uma comunicação de forma segura, é preciso garantir que algumas propriedades de segurança sejam atendidas pelo sistema criptográfico utilizado. A depender do nível de segurança que se deseja alcançar e do cenário por onde vai trafegar a comunicação, se faz necessária a garantia das seguintes propriedades:

\footnotetext{
${ }^{1}$ Do inglês Local Area Network.
} 
- Autenticidade: garantia de que os participantes de uma comunicação são autênticos, ou seja, um intruso não deve se passar por outro usuário.

- Confidencialidade: garantia de que todas as informações trafegadas ou armazenadas em um sistema de computação sejam acessadas ou manipuladas apenas por usuários autorizados.

- Integridade: garantia de que a informação trafegada durante a comunicação chegará ao seu destino exatamente da mesma forma que partiu da origem, isto é, nenhum dado pode ser adulterado.

- Irretratabilidade (ou não repúdio): garantia de que nem o remetente nem o receptor das informações possam negar, posteriormente, sua transmissão, recepção ou posse.

Algumas aplicações podem requerer propriedades de segurança adicionais, como por exemplo anonimato dos participantes da comunicação, ou controle de acesso (restringir o acesso de recursos à entidades privilegiadas). A descrição dessas e de outras propriedades de segurança vão além do escopo deste trabalho. Em Menezes et al. (1996) é possível encontrar outras propriedades com mais detalhes.

\subsection{Criptografia de chave secreta}

A criptografia de chave secreta, também chamada de criptografia simétrica, é a forma mais tradicional de criptografia. Nela, duas entidades $A$ e $B$ compartilham uma única chave secreta $k$ para cifrar e decifrar mensagens. Sua principal característica é a necessidade de um canal de comunicação totalmente seguro, o qual será utilizado para transmitir a chave que será usada nas futuras comunicações cifradas.

Outra característica relevante é que o número de chaves necessárias cresce em ordem de grandeza proporcional ao quadrado do número de participantes da comunicação, uma vez que a chave utilizada por cada par de usuários é única, e não pode ser usada por um terceiro usuário. Para um universo de $n$ participantes, podemos imaginar um grafo com $n$ vértices, representando cada participante, em que cada par distinto de vértices está ligado por uma única aresta, e cada uma dessas arestas representa uma chave secreta. Assim sendo, o número de chaves necessárias para que $n$ participantes possam se comunicar é igual ao número de arestas em um grafo completo com $n$ vértices, portanto:

$$
\text { Quantidade de chaves necessárias }=\frac{n(n-1)}{2}
$$

É importante ressaltar que não existe a propriedade de irretratabilidade em sistemas de criptografia simétrica, pois como os dois extremos da comunicação compartilham a mesma chave secreta, é impossível distinguir qual dos dois criptografou determinada mensagem.

Uma das vantagens da criptografia simétrica é que para um mesmo nível de segurança, seus algoritmos são, em geral, mais rápidos do que algoritmos de criptografia assimétrica. Dentre os algoritmos de criptografia simétrica mais difundidos, estão o DES (Data Encription Standard) e o AES (Advanced Encription Standard).

\subsection{Criptografia de chave pública}

A criptografia de chave pública, também conhecida como criptografia assimétrica, teve origem a partir da publicação do artigo New Directions in Cryptography (Diffie e Hellman, 1976). Nesse artigo foi apresentado o primeiro mecanismo de acordo de chave, onde dois participantes de uma comunicação poderiam "combinar" uma chave secreta usando somente um canal público.

Com base no modelo proposto por Diffie e Hellman, um mecanismo mais sofisticado foi publicado em Rivest et al. (1978), que introduziu o algoritmo RSA, cujas iniciais remetem a seus criadores: Rivest, Shamir e Adleman. Além do algoritmo RSA, existem outros esquemas de criptografia de 
chave pública, dentre os quais o algoritmo ElGamal, os algoritmos baseados em curvas elípticas, e os algoritmos baseados em emparelhamentos bilineares sobre curvas elípticas. Esses últimos serão estudados mais detalhadamente no decorrer deste trabalho.

Nos criptossistemas de chave pública, cada participante da comunicação possui um par de chaves $(S, P)$ relacionadas matematicamente, sendo $S$ sua chave particular, que deve ser mantida secreta, e $P$ sua chave pública, que deve ser conhecida por todos os participantes da comunicação. Dessa forma, é possível suplantar o problema da distribuição de chaves inerente à criptografia simétrica, haja vista que não há necessidade de um canal seguro para distribuição das chaves públicas.

Criptossistemas de chave pública são baseados em funções matemáticas chamadas funções unidirecionais com segredo ${ }^{2}$. Uma função unidirecional é uma função matemática computacionalmente fácil de ser calculada em uma direção, mas computacionalmente difícil de ser calculada na direção oposta. Uma função unidirecional com segredo, por sua vez, é uma função unidirecional cuja inversa pode ser facilmente calculada dada uma informação (trapdoor), e difícil sem essa informação.

Tanto a chave privada $S$ como a chave pública $P$ são utilizadas para criptografar e decriptografar mensagens. Suponha que $x$ denote um texto legível e $S($ ) denote a aplicação da chave privada $S$, que transforma $x$ em $S(x)=y$. Sendo $P()$ a aplicação da chave pública $P$, tem-se que $P(y)=x$, ou seja, a chave $S$ é a inversa da chave $P$, i.e., $P(S(x))=x$ (Terada, 2008).

Uma consequência da existência das chaves $(S, P)$, é que quando utilizamos a chave pública $P$ de uma entidade (digamos Alice) para criptografar uma mensagem, garantimos a autenticidade no destino, pois somente Alice, com sua chave privada $S$, pode recuperar a mensagem original. Em contrapartida, quando Alice utiliza sua chave privada $S$ para criptografar uma mensagem, é garantida a autenticidade na origem, pois somente Alice, que possui a chave privada $S$, poderia ter produzido o texto cifrado. Para garantir a autenticidade em ambos os extremos da comunicação, é necessário empregar um esquema combinado de criptografia e assinatura (Júnior, 2003).

\subsubsection{Assinatura digital}

Assinaturas digitais fornecem um meio de vincular a identidade de uma entidade a um pedaço de informação enviada por ela. O processo de assinar implica em transformar uma mensagem e alguma informação secreta de posse do remetente dessa mensagem em uma espécie de "marca" única, denominada assinatura (Menezes et al., 1996).

Através de assinaturas digitais o receptor da mensagem terá a certeza de que ela foi enviada por quem realmente diz tê-la enviado, garantindo autenticação da origem. Também há garantia de que a mensagem não foi alterada por alguma entidade mal-intencionada ou alguma falha na linha de comunicação durante sua transmissão, garantindo integridade da mensagem.

Para definir genericamente o processo de assinatura digital, considere:

- $\mathcal{M}$ o conjunto de todas as mensagens que podem ser assinadas.

- $\mathcal{S}$ o conjunto de todas as cadeias de caracteres de tamanho fixo, chamadas de assinaturas.

- $S_{A}()$ uma transformação do conjunto de mensagens $\mathcal{M}$ para o conjunto de assinaturas $\mathcal{S}$.

- $V_{A}()$ uma transformação do conjunto $\mathcal{M} \times \mathcal{S}$ para o conjunto \{verdadeiro, falso $\}$.

Suponhamos que a entidade $A$ deseja enviar uma mensagem $m$, assinada por ela, para a entidade $B$. A entidade $A$ deve, então:

1. Calcular: $s=S_{A}(m)$.

2. Transmitir o par $(m, s)$ para B. $s$ é a assinatura da mensagem $m$.

Na outra ponta da comunicação, $B$ recebe $(m, s)$ e deseja verificar sua autenticidade. $B$ deve:

\footnotetext{
${ }^{2}$ Do inglês trapdoor one-way function.
} 
1. Calcular: $u=V_{A}(m, s)$.

2. Aceitar a assinatura como sido criada por $A$ se $u=$ verdadeiro, ou rejeitar a assinatura se $u=$ falso.

Deve ser computacionalmente inviável para qualquer outra entidade que não seja $A$, calcular uma mensagem $m$ e alguma cadeia de caracteres $s$ tal que $V_{A}(m, s)=$ verdadeiro. Na prática, a transformação $S_{A}()$ é implementada utilizando-se funções de $h a s h^{3}$ criptográficas.

É importante ressaltar que o uso de assinaturas digitais só é possível na criptografia assimétrica, uma vez que na criptografia simétrica, como todos os participantes da comunicação compartilham a mesma chave, não é possível determinar qual participante enviou a mensagem.

\subsubsection{Infraestrutura de chaves públicas}

Para que seja possível a comunicação entre as entidades $A$ e $B$ utilizando criptografia assimétrica, é necessário que o remetente da mensagem conheça a chave pública da entidade com a qual ele deseja se comunicar. Entretanto, como as chaves públicas são disseminadas em um canal de comunicação público (não seguro), tais chaves podem ser falsificadas por um impostor, isto é, uma entidade $C$ mal-intencionada, possibilitando uma ofensiva conhecida na literatura como ataque man-in-themiddle (ou ataque do "homem no meio"). Esse falso usuário personifica o receptor perante o emissor e ainda se faz passar pelo emissor perante o receptor. Dessa forma, ele é capaz de decifrar todas as mensagens trocadas entre $A$ e $B$ (Goya, 2006).

Devido à necessidade de um mecanismo para autenticar chaves públicas, criou-se o conceito de certificado digital. De maneira simplificada, um certificado digital é análogo a um documento registrado em cartório, que comprova que uma chave pública realmente pertence à determinada entidade. O cartório, neste caso, é representado por uma entidade jurídica, que possui fé pública, chamada de autoridade certificadora $(\mathrm{AC})$.

O certificado digital é um documento padronizado (X-509), assinado por uma autoridade certificadora, e contém os seguintes campos principais:

- Versão do certificado

- Nome da instituição

- Chave pública da instituição

- Nome da entidade certificadora

- Data de emissão

- Data de expiração

- Número de série

- Algoritmo utilizado para assinar

Na figura 2.1 é exibido um certificado digital retirado do sítio http://webmail.ime.usp.br, obtido no dia 28/02/2012.

\footnotetext{
${ }^{3}$ Para maiores informações sobre funções de hash (ou espalhamento), consultar Terada (2008).
} 

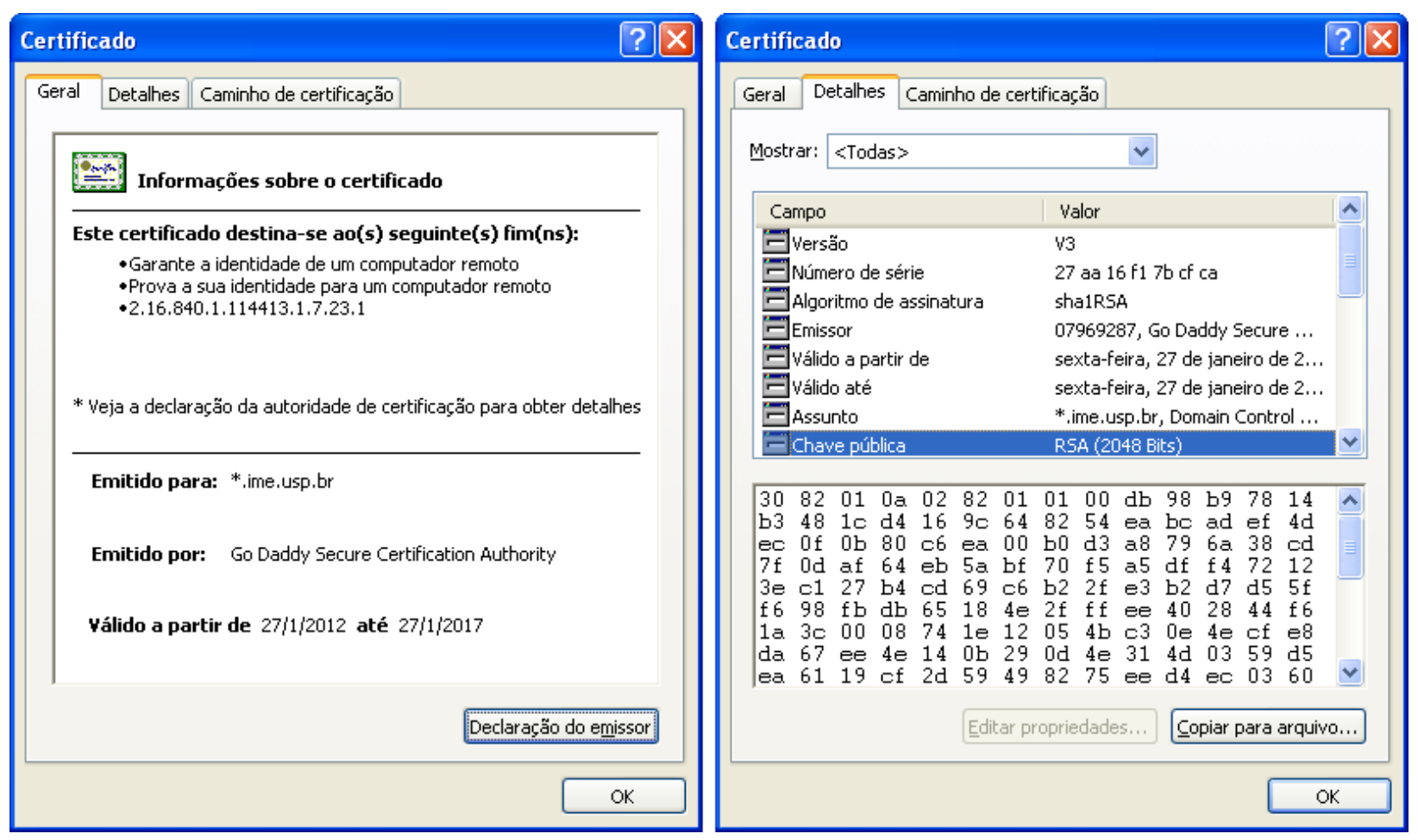

Figura 2.1: Exemplo de certificado digital.

Na prática, uma autoridade certificadora é um servidor que administra uma base de dados que associa uma dada chave pública aos atributos do seu proprietário. Dependendo do tamanho do sistema, pode ser necessário haver mais de uma autoridade certificadora, formando um conjunto distribuído de servidores, chamado infra-estrutura de chaves públicas (ICP ou PKI - Public Key Infrastructure) (Terada, 2008). Essas autoridades certificadoras são organizadas hierarquicamente, formando um modelo de certificação. Os dois modelos de certificação mais comuns são os modelos em árvore e em floresta de árvores. Como pode ser visto na figura 2.2, existe uma autoridade certificadora raiz, que certifica ela mesma. Outras autoridades certificadoras estão subordinadas à autoridade raiz e também podem certificar outras autoridades certificadoras subordinadas a elas.

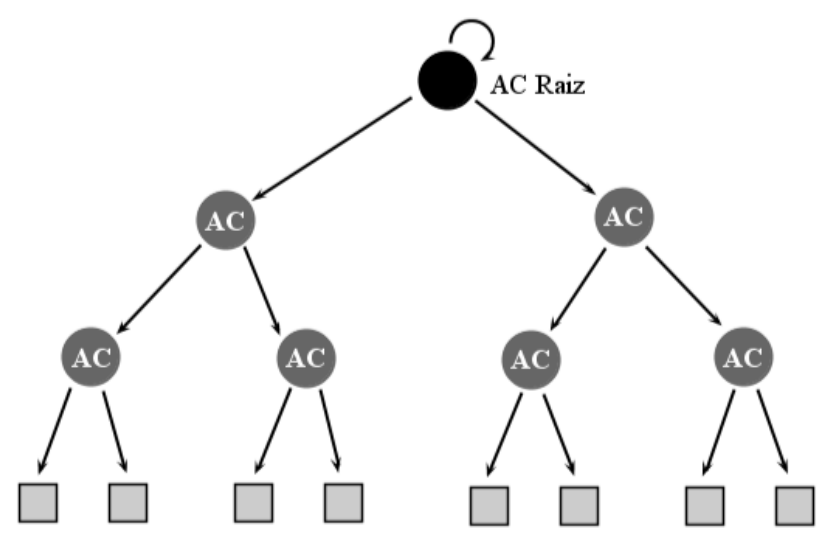

Figura 2.2: Modelo de certificação em árvore.

No modelo em floresta de árvores, mostrado na figura 2.3, existem várias autoridades raiz que se comunicam, e cada uma é raiz de sua árvore de certificação. 


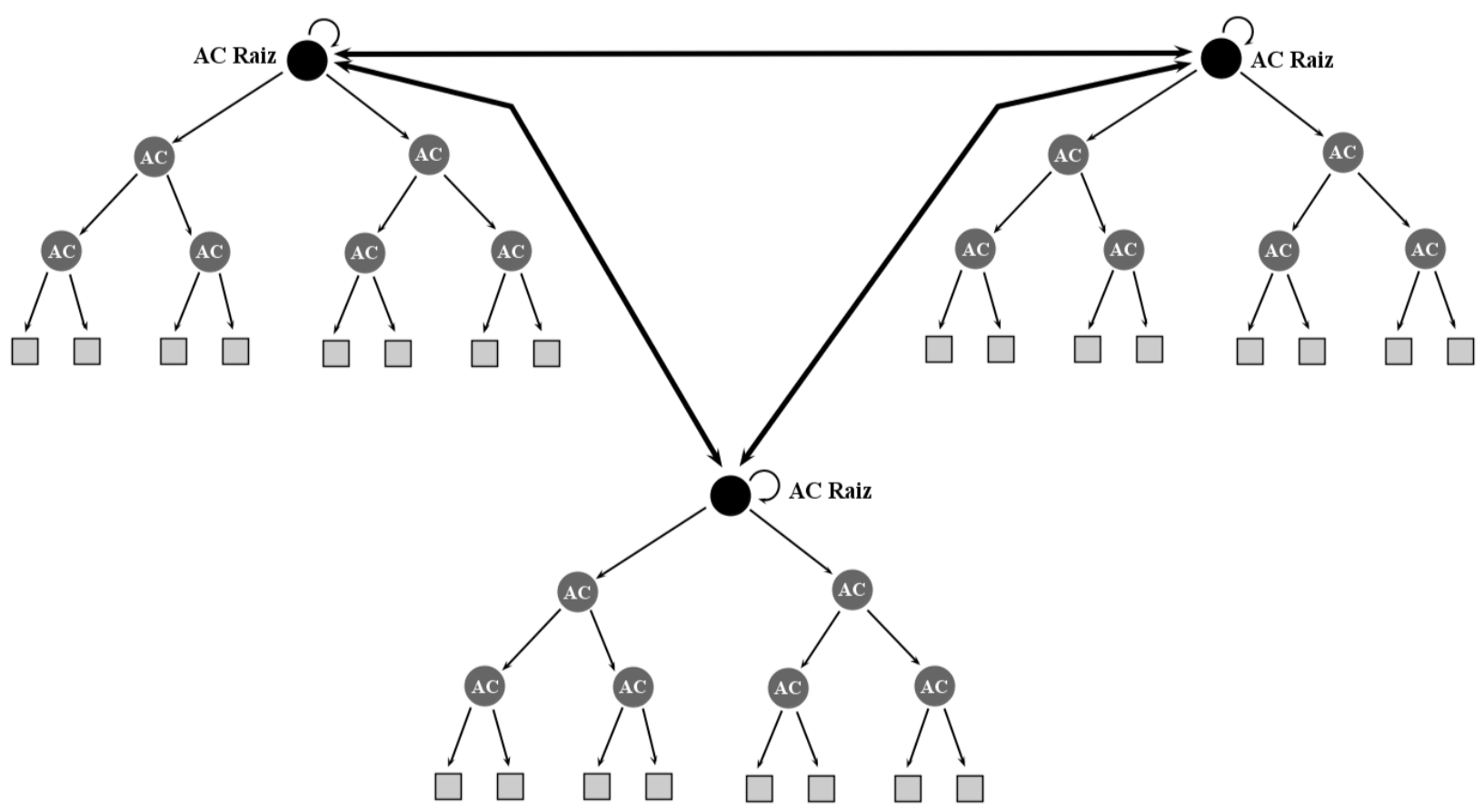

Figura 2.3: Modelo de certificação em floresta.

Um dos grandes desafios de implantação de uma infra-estrutura de chaves públicas é que toda hierarquia de certificação depende do certificado da autoridade raiz ser obtido através de um canal confiável e legítimo por todas as partes interessadas (Júnior, 2003). 


\section{Capítulo 3}

\section{Emparelhamentos Bilineares}

Este capítulo apresentada uma introdução aos emparelhamentos bilineares e problemas computacionais relacionados, considerando as definições apresentadas no Apêndice A.

Dados três grupos $\mathbb{G}_{1}, \mathbb{G}_{2}$ e $\mathbb{G}_{T}$ de ordem prima $q$, tal que $\mathbb{G}_{1}$ e $\mathbb{G}_{2}$ são grupos aditivos e $\mathbb{G}_{T}$ é um grupo multiplicativo, um emparelhamento bilinear é definido como um mapeamento $e: \mathbb{G}_{1} \times \mathbb{G}_{2} \rightarrow \mathbb{G}_{T}$ que possui as seguintes propriedades (considere $P, R \in \mathbb{G}_{1}, Q, S \in \mathbb{G}_{2}$ e $\left.a, b \in \mathbb{Z}_{q}\right)$ :

1. Bilinearidade: um emparelhamento $e$ é dito bilinear se valem as seguintes propriedades:

(a) Propriedade distributiva em relação ao primeiro argumento

$$
e(P+R, Q)=e(P, Q) \cdot e(R, Q)
$$

(b) Propriedade distributiva em relação ao segundo argumento

$$
e(P, Q+S)=e(P, Q) \cdot e(P, S)
$$

(c) Das propriedades 3.1 e 3.2 , tem-se que

$$
e(a P, b Q)=e(P, b Q)^{a}=e(a P, Q)^{b}=e(P, Q)^{a b}
$$

A Figura 3.1 ilustra um mapeamento bilinear.

2. Não-degeneração: um emparelhamento $e$ é dito não-degenerado se não mapeia todos os pares $\mathbb{G}_{1} \times \mathbb{G}_{2}$ para o elemento identidade de $\mathbb{G}_{T}$. Como $\mathbb{G}_{1}$ e $\mathbb{G}_{2}$ são grupos de ordem prima $q$, se $P$ é um gerador de $\mathbb{G}_{1}$, e $Q$ é um gerador de $\mathbb{G}_{2}$, então $e(P, Q)$ é um gerador de $\mathbb{G}_{T}$.

3. Computabilidade: um mapeamento é computável se existe um algoritmo eficiente (i.e., de tempo polinomial), para calcular $e(P, Q)$, para todo $P \in \mathbb{G}_{1}$ e $Q \in \mathbb{G}_{2}$. 


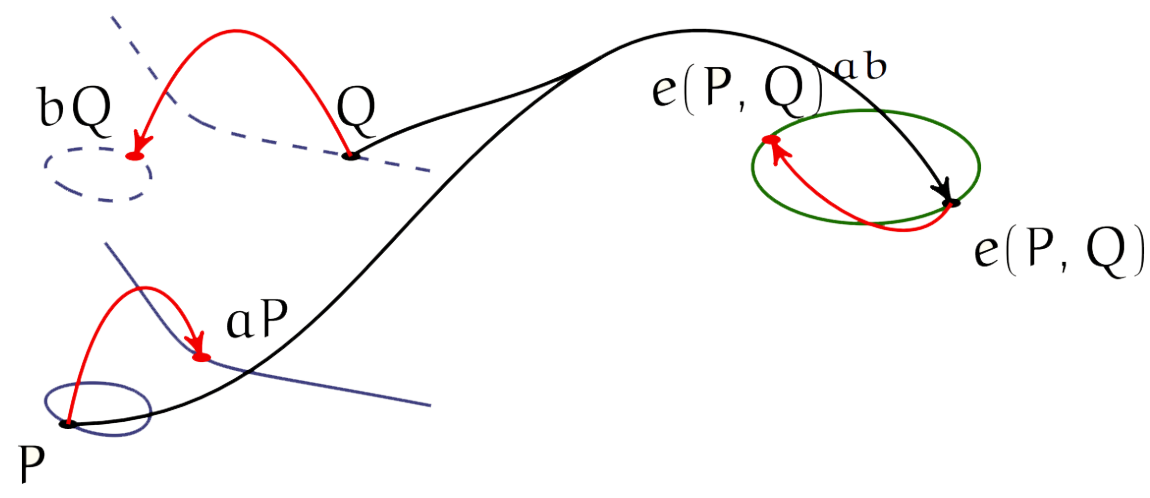

Figura 3.1: Ilustração de um emparelhamento Bilinear (Cesena, 2010).

Em Galbraith et al. (2006) são discutidos três tipos de emparelhamentos bilineares:

- Emparelhamentos do Tipo 1 são um caso especial da definição apresentada neste trabalho, onde $\mathbb{G}_{1}=\mathbb{G}_{2}$, i.e., e : $\mathbb{G}_{1} \times \mathbb{G}_{1} \rightarrow \mathbb{G}_{T}$, denominados emparelhamentos simétricos. Em geral são implementados sobre curvas supersingulares, e existe algoritmo de hash criptográfico eficiente que mapeia cadeias de bits aleatórias em $\mathbb{G}_{1}$.

- Emparelhamentos do Tipo 2 e do Tipo 3 são denominados de emparelhamentos assimétricos e são implementados sobre curvas ordinárias. A diferença entre ambos é que no Tipo 2 existe um homomorfismo $\phi: \mathbb{G}_{2} \rightarrow \mathbb{G}_{1}$ que pode ser computado de maneira eficiente, e para emparelhamentos do Tipo 3 não se conhece nenhum homomorfismo entre $\mathbb{G}_{1}$ e $\mathbb{G}_{2}$ eficientemente computável.

Para emparelhamentos do Tipo 2 e do Tipo 3 existe algoritmo de hash criptográfico eficiente que mapeia cadeias de bits aleatórias em $\mathbb{G}_{1}$. Entretanto para emparelhamentos do Tipo 2 não existe tal algoritmo de hash eficiente que mapeie em $\mathbb{G}_{2}$. Já para os emparelhamentos do Tipo 3 existe algoritmo de hash, não tão eficiente quanto o que mapeia em $\mathbb{G}_{1}$, para mapear cadeias de bits aleatórias em $\mathbb{G}_{2}$.

\subsection{Complexidade de algoritmos e problemas computacionais de interesse}

Considere as funções $f(n)$ e $g(n)$ definidas sobre inteiros $n>0$, com valores reais $f(n)>0$ e $g(n)>0$, para infinitos valores de $n$. Dizemos que $f(n)=O(g(n))$ se existem uma constante $c>0$ e um inteiro $n_{0}$, tais que $0 \leq f(n) \leq c g(n)$, para todo $n \geq n_{0}$. Ou seja, para $n$ suficientemente grande, $f(n)$ não cresce mais que $g(n)$, a menos de um fator constante. A notação $O(\star)$ é conhecida como notação assintótica (Cormen et al., 2009).

Seja $n$ o comprimento dos dados de entrada para um algoritmo $\mathcal{A}$, e $f(n)$ a função do tempo de execução de $\mathcal{A}$, no pior caso (em outras palavras, o tempo de execução de $\mathcal{A}$ é limitado superiormente por $f(n))$. Diz-se que $\mathcal{A}$ possui complexidade de tempo polinomial, quando $f(n)=O\left(n^{k}\right)$, para uma constante $k$. Para o caso em que $f(n)=O\left(k^{n}\right)$, dizemos que $\mathcal{A}$ possui complexidade de tempo exponencial, para uma constante $k>1$.

Um problema é considerado difícil ${ }^{1}$, quando não se conhece qualquer algoritmo que possa resolvêlo em tempo polinomial. Alguns desses problemas são de grande interesse para a criptografia, como, por exemplo, o problema da fatoração de inteiros, utilizado no RSA, e o problema do logaritmo discreto, que será apresentado nas Seções 3.1.1 e 3.1.2.

\footnotetext{
${ }^{1}$ Computacionalmente ineficiente, inviável, intratável.
} 


\subsubsection{Problema do logaritmo discreto}

Dados um grupo cíclico $\mathbb{G}$ de ordem $q$, e um gerador $g \in \mathbb{G}$, tem-se que $\left\{g^{0}, g^{1}, \ldots, g^{q-1}\right\}=\mathbb{G}$, i.e., para cada $h \in \mathbb{G}$ existe um único $x \in \mathbb{Z}_{q}$, tal que $h=g^{x}$. Em outra notação, podemos afirmar que $x=\log _{g} h$. Logo, dizemos que $x$ é o logaritmo discreto ${ }^{2}$ de $h$ na base $g$.

O problema do logaritmo discreto (DLP - Discrete Logarithm Problem) consiste em encontrar o valor de $x$, dados os parâmetros públicos $(\mathbb{G}, g, h)$. Quando o tamanho do grupo é relativamente longo, até hoje não se conhece um algoritmo eficiente, i.e., de tempo polinomial, para resolver este problema (Terada, 2008).

O primeiro sistema criptográfico cuja segurança se baseava no problema do logaritmo discreto foi o protocolo de acordo de chaves proposto em Diffie e Hellman (1976). Em 1984, ElGamal propôs um esquema de criptografia de chave pública e assinatura com segurança baseada nesse problema.

\subsubsection{Problema do logaritmo discreto sobre curvas elípticas}

O problema do logaritmo discreto pode ser generalizado, considerando-se qualquer grupo finito $\mathcal{G}$ com uma operação o. Dados $A \in \mathcal{G}$ um gerador do subgrupo $\mathcal{W} \subseteq \mathcal{G}$, e $B \in \mathcal{W}$, o problema do logaritmo discreto geral consiste em calcular um inteiro $s: 1 \leq s \leq|\mathcal{W}|-1$, tal que $A^{s}=B$, onde $A^{s}=\underbrace{A \mathfrak{o} A \mathfrak{o} \ldots \mathfrak{o} A}_{s \text { vezes }}$ (Terada, 2008).

No caso de $\stackrel{s}{\mathcal{G}}$ ser o conjunto de pontos de uma curva elíptica $E$ sobre um corpo finito $\mathcal{K}$, a operação o é definida como a soma de dois pontos de $E(\mathcal{K})$, isto é, $\mathcal{G}$ é escrito em notação aditiva. O problema do logaritmo discreto sobre curvas elípticas consiste em dados os pontos $P, Q \in E(\mathcal{K})$, calcular um inteiro $s$ tal que $Q=s P$.

\subsubsection{Outros problemas relacionados}

A partir do problema do logaritmo discreto (conforme as definições das Seções 3.1.1 e 3.1.2) surgiram outros problemas relacionados, conhecidos como problemas Diffie-Hellman. Considere $\mathcal{G}$ um grupo aditivo e $\mathcal{G}^{\prime}$ um grupo multiplicativo, ambos de ordem prima $q$.

- Problema Diffie-Hellman de decisão (DDH - Decision Diffie-Hellman problem): dados $a, b, c, n \in$ $\mathbb{Z}_{q}$ e $P, a P, b P, c P \in \mathcal{G}$, decidir se $c=a b \bmod n$. Para o caso bilinear (DBDH - Decision Bilinear Diffie-Hellman problem), dados $P, a P, b P, c P \in \mathcal{G}$ e $T \in \mathcal{G}_{T}$, decidir se $e(P, P)^{a b c} \stackrel{?}{=} T$.

Note que se existe um mapeamento bilinear não-degenerado $e: \mathcal{G} \times \mathcal{G} \rightarrow \mathcal{G}^{\prime}$, então o DDHP pode ser resolvido de maneira eficiente, uma vez que $c=a b \bmod n$ se, e somente se, $e(P, c P)=$ $e(a P, b P)$.

- Problema Diffie-Hellman computacional (CDH - Computational Diffie-Hellman problem): da$\operatorname{dos} a, b \in \mathbb{Z}_{q}$ e $P, a P, b P \in \mathcal{G}$, calcular $a b P$.

- Problema Diffie-Hellman lacunar (Gap-DH - Gap Diffie-Hellman problem): consiste em resolver o problema $\mathrm{CDH}$ em $\mathcal{G}$, com ajuda de um oráculo que resolve o problema DDH em $\mathcal{G}$. Para o caso bilinear (Gap-BDH ou GBDH - Gap-Bilinear Diffie-Hellman problem), dados $P, a P, b P, c P \in \mathcal{G}$, calcular $e(P, P)^{a b c}$, com ajuda de um oráculo de decisão DBDH.

- Problema Diffie-Hellman bilinear (BDH - Bilinear Diffie-Hellman problem): dados $a, b, c \in \mathbb{Z}_{q}$ e $P, a P, b P, c P \in \mathcal{G}$, calcular $e(P, P)^{a b c}$.

A família de problemas lacunares foi apresentada por Okamoto e Pointcheval (2001). Já o problema BDH foi definido por Boneh e Franklin (2003). Do ponto de vista teórico, um protocolo que tenha sido demonstrado seguro sob a hipótese de dificuldade do problema BDH é, pelo menos, tão seguro quanto outro que tenha sido demonstrado seguro sob a hipótese de dificuldade do problema

${ }^{2} \hat{E}$ dito logaritmo "discreto" porque seus valores estão definidos em um intervalo finito. 
Gap-BDH, no mesmo modelo de segurança. Por esse motivo, é preferível utilizar protocolos que se apoiem na suposição de dificuldade do problema BDH, e o uso do Gap-BDH deve ser evitado sempre que possível (Goya, 2011).

Para cada um desses problemas, existem também os chamados co-problemas. Considere agora dois grupos aditivos $\mathcal{G}_{1}$ e $\mathcal{G}_{2}$ ao invés de um, e o emparelhamento assimétrico $e: \mathcal{G}_{1} \times \mathcal{G}_{2} \rightarrow \mathcal{G}^{\prime}$.

- co-DDHP (co-Decision Diffie-Hellman Problem): dados $a, b \in \mathbb{Z}_{q}, P, a P \in \mathcal{G}_{1}$ e $Q, b Q \in \mathcal{G}_{2}$, onde $P$ e $Q$ possuem a mesma ordem $r$. O co-DDHP consiste em decidir se $a=b \bmod r$.

- co-CDHP (co-Computational Diffie-Hellman Problem): dados $a \in \mathbb{Z}_{q}, P, a P \in \mathcal{G}_{1}$ e $Q \in \mathcal{G}_{2}$, calcular $a Q$.

- co-GDHP (co-Gap Diffie-Hellman Problem): consiste em resolver o co-CDHP em $\mathcal{G}_{1}$ e $\mathcal{G}_{2}$, com ajuda de um oráculo que resolve o co-DDHP nesses grupos.

- co-BDHP (co-Bilinear Diffie-Hellman Problem): dados $a, b \in \mathbb{Z}_{q}, P, a P, b P \in \mathcal{G}_{1}$ e $Q \in \mathcal{G}_{2}$, calcular $e(P, Q)^{a b}$. 


\section{Capítulo 4}

\section{Modelos Alternativos de Criptografia de Chave Pública}

Neste capítulo serão estudadas algumas variantes do modelo de criptografia de chave pública que eliminam as dificuldades impostas por uma infraestrutura de chaves públicas (ICP), como ocorre na criptografia assimétrica tradicional. Na sequência são discutidos protocolos de acordo de chave, atributos e modelos de segurança.

\subsection{Modelo de chave pública baseado em identidade}

O conceito de criptografia baseada em identidades foi originalmente proposto por Shamir (1984). Esse artigo propunha um novo modelo criptográfico, no qual não seria necessária a troca de chaves secretas entre os participantes de uma comunicação, como ocorre na criptografia simétrica. Também não há necessidade de manter um diretório de chaves públicas e nem de certificados digitais para garantir a autenticidade de tais chaves públicas, como acontece na criptografia assimétrica tradicional.

Nesse novo modelo, Shamir (1984) pressupunha a existência de um Gerador de Chaves Privadas (PKG - Private Key Generator), cuja função é gerar uma chave particular para um usuário solicitante, e entregá-la através de um canal de comunicação seguro. No artigo, a ideia original era que essa chave particular seria entregue em uma espécie de cartão inteligente (smart card) personalizado.

Diferente da criptografia assimétrica tradicional, na qual o par de chaves é gerado aleatoriamente e não tem nenhuma ligação com seu dono, a chave pública no modelo baseado em identidades deve ser um identificador do usuário. Tal identificador pode ser, por exemplo, o nome do usuário, CPF, endereço de e-mail, endereço de rede, número de telefone, ou uma combinação de dois ou mais desses identificadores, desde que identifique unicamente o usuário e que seu portador não possa negar que determinada chave pública é sua posteriormente. Além disso, após gerar e entregar as chaves particulares a seus respectivos usuários, o PKG não precisa mais participar da comunicação, permitindo que a rede funcione de forma completamente descentralizada por um período indefinido de tempo.

Shamir também notou que esse tipo de criptografia permitiria a criação de um sistema de email ideal. Se uma usuária Alice sabe o endereço eletrônico de Beto, ela pode enviar mensagens para Beto que apenas ele possa ler, e Alice pode verificar assinaturas que apenas Beto poderia ter produzido. Isso faria com que os aspectos criptográficos da comunicação ficassem mais transparentes para os usuários, permitindo que o sistema seja utilizado de forma eficiente mesmo por usuários sem conhecimentos sobre chaves e protocolos. Uma vantagem clara desse tipo de sistema sobre a criptografia assimétrica tradicional é que, como um identificador de usuário não é mais um número aleatório, como é o caso do RSA, um usuário Beto não precisa reservar espaço adicional para armazenar as chaves públicas dos usuários com quem deseja se comunicar. Em vez disso, poderia ser utilizada a própria lista de endereços eletrônicos, por exemplo. 
As chaves privadas no modelo baseado em identidades precisam ser necessariamente computadas pelo PKG, pois não há nada de secreto na identidade dos usuários. Se fosse possível que um usuário Beto computasse sua própria chave privada, então Beto também poderia computar as chaves privadas de outros usuários. Dessa maneira o sistema não poderia ser considerado seguro. Somente o PKG possui total controle na geração de chaves particulares, e dessa forma terá conhecimento das chaves privadas de todos os usuários do sistema. Tal característica é chamada de custódia de chaves (key escrow). Observa-se então, que o nível de confiança depositado no PKG é muito maior do que em esquemas de criptografia assimétrica tradicional.

A Figura 4.1 ilustra como ocorre a geração e distribuição de chaves no modelo baseado em identidade. Seja $s \in \mathbb{Z}_{q}^{*}$ um valor secreto calculado pelo PKG, e sejam $P$ um ponto gerador de $E\left(\mathbb{F}_{q}^{k}\right)$, e $\mathcal{H}$ uma função de hash criptográfico tal que $\mathcal{H}:\{0,1\}^{*} \rightarrow E\left(\mathbb{F}_{q}^{k}\right)$, parâmetros públicos. Chama-se $s$ de chave mestra secreta, pois todas as chaves secretas do sistema são derivadas dela.

\section{PKG}

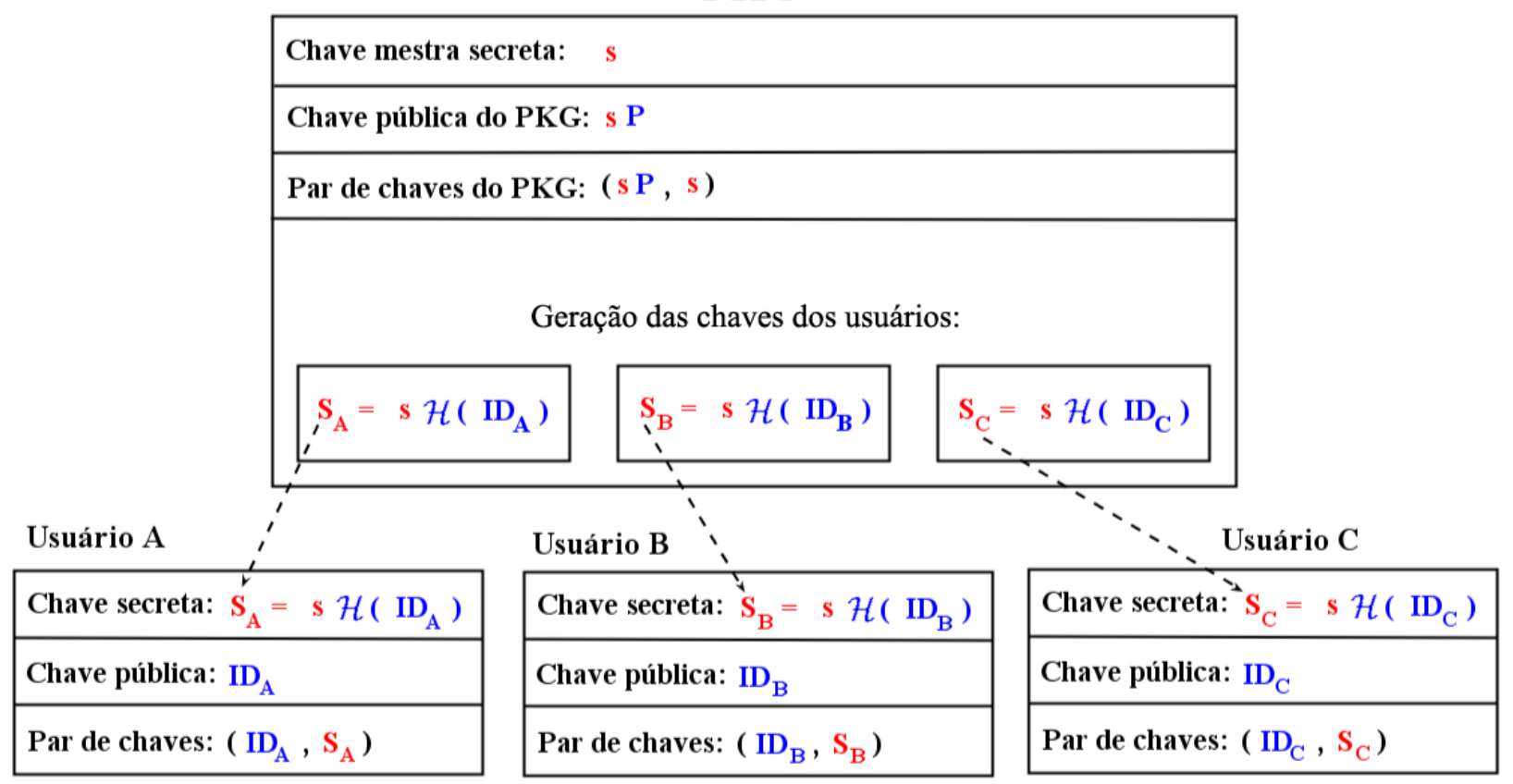

Figura 4.1: Distribução de chaves na criptografia de chave pública baseada em identidade.

Desde a publicação de Shamir (1984), vários pesquisadores haviam tentado, sem sucesso, desenvolver um esquema de criptografia baseado em identidades. No entanto, no ano 2001 foram propostas soluções satisfatórias por Boneh e Franklin (2001), através do uso de emparelhamentos bilineares sobre curvas elípticas, e por Cocks (2001), baseada em resíduos quadráticos. Atualmente, a grande maioria dos esquemas criptográficos baseados em identidades fazem uso de emparelhamentos bilineares sobre curvas elípticas.

\subsection{Modelo de chave pública sem certificado}

O modelo de criptografia de chave pública sem certificado foi proposto originalmente por Al-Riyami e Paterson (2003). Os autores buscavam uma forma de eliminar a propriedade de custódia de chaves, inerente ao modelo baseado em identidade. Nesse artigo foi apresentado um novo protocolo de criptografia ${ }^{1}$ baseado no protocolo de IBE (Identity-Based Encryption) proposto por Boneh e Franklin (2001).

O modelo sem certificado, assim como no modelo baseado em identidade, pressupõe a existência de uma autoridade de confiança, que é responsável por calcular algumas das chaves do sistema.

\footnotetext{
${ }^{1}$ No sentido de encriptação.
} 
Diferente do modelo baseado em identidade, no qual a autoridade de confiança calcula todas as chaves privadas do sistema (implicando na custódia de chaves), nos criptossistemas sem certificado a autoridade de confiança calcula somente parte da chave secreta dos usuários, que chamaremos de chave privada parcial. A outra parte da chave é calculada pelo próprio usuário. Através do uso de uma função matemática que recebe como entrada as duas chaves privadas parciais, o usuário consegue calcular sua chave particular completa.

Como a autoridade de confiança do sistema não calcula a chave privada completa dos usuários, tal autoridade é chamada centro gerador de chaves (KGC - Key Generation Center). O KGC funciona de forma muito semelhante ao PKG da criptografia de chave pública baseada em identidade. A diferença está no lado do usuário.

Cada participante $U$ escolhe um valor secreto $x_{U} \in \mathbb{Z}_{q}^{*}$. Este segredo fará parte tanto da sua chave secreta $S_{U}$, tanto de sua chave pública $X_{U}$. O KGC calcula a chave secreta parcial $D_{U}$ e envia para o participante $U$ por um canal seguro. A Figura 4.2 ilustra como ocorre a geração e distribuição das chaves secretas parciais no modelo sem certificado, e como cada usuário $U$ calcula sua chave secreta completa e sua chave pública.

\begin{tabular}{|c|c|c|c|c|c|}
\hline & & & & & \\
\hline & Chave mestra & creta: & & & \\
\hline & Chave pública & $\mathrm{PKG}$ & & & \\
\hline & Par de chaves & PKG & $\mathrm{sP}$, & & \\
\hline & & & ão d & & \\
\hline & $\mathrm{D}_{\mathrm{A}}=\mathrm{s} \mathcal{H}$ & $\left.D_{A}\right)$ & & $\mathrm{s} \mathcal{H}\left(\mathrm{ID}_{\mathrm{C}}\right)$ & \\
\hline Usuário & & & ário & & suário $\mathrm{C}$ \\
\hline Chave secreta 1 & $\mathrm{~s} \mathcal{H}\left(\mathrm{ID}_{\mathrm{A}}\right)$ & \begin{tabular}{|l|} 
Chave \\
\end{tabular} & reta p & \begin{tabular}{|l|} 
Chave secreta \\
\end{tabular} & $\mathrm{D}_{\mathrm{C}}=\mathrm{s} \mathcal{H}\left(\mathrm{ID}_{\mathrm{C}}\right)$ \\
\hline Segredo de A: & & Segres & le $A:$ & Segredo de A: & $\mathrm{x}_{\mathrm{C}}$ \\
\hline Chave secreta c & $\mathrm{x}_{\mathrm{A}} \mathrm{D}_{\mathrm{A}}$ & Chave & reta c & Chave secreta c & a: $S_{C}=x_{C} D_{C}$ \\
\hline Chave pública: & $\mathrm{x}_{\mathrm{A}} \mathrm{P}$ & Chave & blica: & Chave pública: & $X_{C}=x_{C} P$ \\
\hline Par de chaves: & $\left., \mathrm{ID}_{\mathrm{A}} \mathrm{l}, \mathrm{S}_{\mathrm{A}}\right)$ & Par de & aves: & Par de chaves: & $\left(\left[\mathrm{X}_{\mathrm{C}}, \mathrm{ID}_{\mathrm{C}}\right], \mathrm{s}_{\mathrm{C}}\right)$ \\
\hline
\end{tabular}

Figura 4.2: Distribução de chaves na criptografia de chave pública sem certificado.

Além da vantagem da eliminação da custódia de chaves, esse modelo apresenta outras vantagens. O risco de comprometimento da chave mestra secreta é menor do que no modelo baseado em identidade, pois caso haja alguma violação dela, somente chaves parciais serão comprometidas. Para conseguir decifrar mensagens cifradas anteriormente ao comprometimento da chave mestra secreta, seria necessário obter o segredo dos usuários também. Portanto, o nível de confiança depositado no KGC é menor do que nos criptossistemas baseados em identidade. Já para personificar usuários ou decifrar novas mensagens, antes será necessário que o adversário substitua as chaves públicas por valores cujo segredo associado seja escolhido por ele. Como consequência da possibilidade desse ataque, é importante ressaltar que as chaves privadas parciais devem ser entregues aos usuários pelo KGC através de um canal seguro e autenticado.

Uma outra vantagem importante e exclusiva desse modelo é que o processo de renovação da chave pública pode ser totalmente controlado pelo usuário. O usuário pode atualizar ou até mesmo possuir várias chaves públicas, sem a necessidade de se comunicar com o KGC. De maneira semelhante ao modelo baseado em identidade, um usuário pode criar uma chave pública e utilizá-la (somente para 
cifrar) antes de possuir uma chave privada. Ao obter sua chave secreta parcial do KGC, o usuário habilitará a decifração.

Uma das desvantagens é a necessidade de um diretório de chaves públicas, pois como a chave pública de cada usuário é composta pela identidade do usuário e por um ponto na curva elíptica $E\left(\mathbb{F}_{q}^{k}\right)$, é necessário guardar este último valor em um repositório público, por não ser facilmente memorizável por humanos. Outra desvantagem é a vulnerabilidade deste modelo para ataques do tipo Denial of Decryption (DOD). Um ataque DOD se dá quando algum usuário mal-intencionado divulga uma chave pública falsa no repositório de chaves públicas, com objetivo de prejudicar um usuário legítimo do sistema. Este usuário ficará incapacitado de decifrar mensagens que lhe tenham sido enviadas, caso estejam cifradas com a chave falsa. De forma análoga, assinaturas digitais deixarão de ser verificáveis, e, num primeiro momento, não será possível detectar se o que está errado é a chave pública ou a assinatura (Goya, 2011).

\subsection{Comprimento das chaves}

Na maioria dos sistemas criptográficos, o tamanho da chave é um importante parâmetro de segurança. Uma das grandes vantagens dos sistemas criptográficos baseados em curvas elípticas (ECC - Elliptic Curve Cryptosystem) em relação aos sistemas de criptografia de chave pública tradicionais, como por exemplo, o RSA, é que parâmetros significativamente menores podem ser usados nos ECCs, mantendo o mesmo nível de segurança, como mostrado na Tabela 4.1.

\begin{tabular}{ccccc}
\hline Segurança (bits) & Algoritmo simétrico & RSA & ECC & Razão de crescimento \\
\hline 80 & Skipjack & 1024 & 160 & $\approx 1: 6$ \\
112 & 3DES & 2048 & 224 & $\approx 1: 9$ \\
128 & AES-128 & 3072 & 256 & $1: 12$ \\
192 & AES-192 & 7680 & 384 & $1: 20$ \\
256 & AES-256 & 15360 & 512 & $1: 30$ \\
\hline
\end{tabular}

Tabela 4.1: Comparação entre comprimentos minimos de chaves (em bits) para diferentes niveis de segurança (Barker et al., 2007).

Chaves mais longas fornecem maior segurança, no entanto, utilizá-las significa que a operação criptográfica vai demorar mais tempo para ser completada e mais espaço será necessário para armazená-las. No trabalho de Dent (2010) são detalhadas as metodologias empregadas na construção de padrões de segurança, como os da Tabela 4.1.

\subsection{Protocolos de acordo de chave}

Protocolos de acordo de chave possibilitam que duas ou mais entidades combinem uma chave secreta através de um canal de comunicação público. Geralmente esta chave é utilizada em conjunto com algoritmos de criptografia simétrica para posterior troca de mensagens, pois esses algoritmos são mais eficientes computacionalmente se comparados aos algoritmos de criptografia assimétrica. Dizemos que uma execução de um protocolo é uma sessão. O segredo compartilhado resultante dessa sessão é chamado de chave de sessão (Swanson e Jao, 2009a).

Um exemplo clássico de protocolo de acordo de chave entre duas entidades é o protocolo DiffieHellman (Diffie e Hellman, 1976), exemplificado na Figura 4.3. Este protocolo está protegido pelo problema computacional Diffie-Hellman (CDHP), contudo pode ser "quebrado" por um intruso ativo que substitui mensagens, através do ataque man-in-the-middle. Esse ataque e uma modificação no protocolo Diffie-Hellman para evitá-lo são discutidos detalhadamente em Terada (2008). 


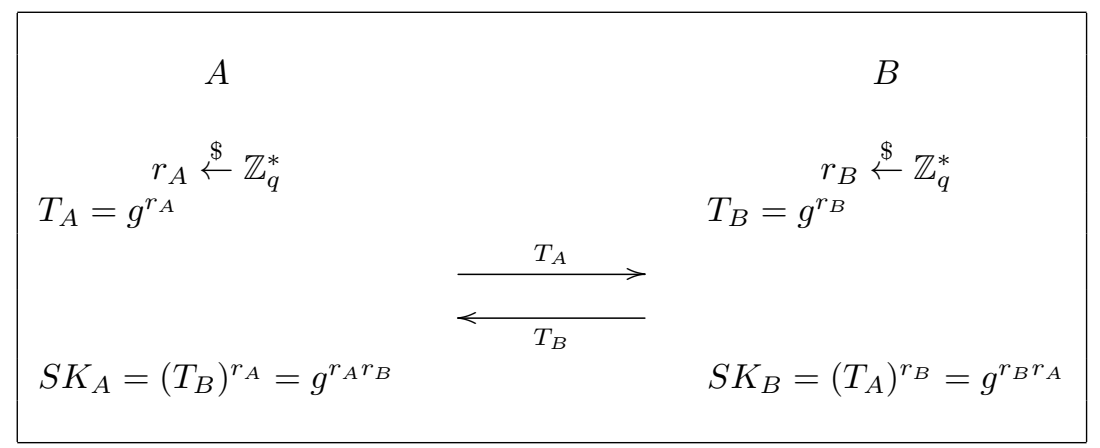

Figura 4.3: Protocolo de acordo de chave Diffie-Hellman.

Segundo Menezes et al. (1996), existem várias propriedades que os protocolos de acordo de chave podem possuir, e dependendo da aplicação, devem prover:

- Autenticação de chave (implícita): quando um participante tem certeza de que apenas um segundo participante, especificamente identificado, consegue acessar a chave de sessão.

- Confirmação de chave: quando um participante pode ter certeza de que um segundo participante (não necessariamente identificado) realmente possui a chave de sessão.

- Autenticação de chave explícita: quando um protocolo possui as propriedades de autenticação de chave implícita e confirmação de chave, dizemos que também possui autenticação de chave explícita.

Para realização deste trabalho, nos interessam os protocolos de acordo de chave com autenticação (conhecidos também por AKA - Authenticated Key Agreement), uma vez que garantem a autenticidade dos participantes.

\subsubsection{Atributos de segurança}

De acordo com Krawczyk (2005) e LaMacchia et al. (2007), os atributos de segurança mais importantes e requeridos em protocolos de acordo de chave com autenticação estão relacionados a seguir:

- Resistência à ataques de personificação básicos: um adversário não deve ser capaz de personificar um usuário se não conhecer sua chave secreta.

- Resistência a ataques de compartilhamento desconhecido de chave (UKS - Unknown Key-Share): deve ser inviável convencer um participante $A$ de que ele está compartilhando uma chave com $B$, quando na realidade está compartilhando com outro participante $C$ (honestamente registrado no sistema), enquanto $C$ pensa (corretamente) estar compartilhando com $A$.

- Segurança de chave desconhecida: cada execução do protocolo deve gerar uma chave de sessão única. O protocolo deve permanecer seguro mesmo que um adversário descubra algumas chaves de sessão negociadas anteriormente.

- Segurança a ataques de personificação pelo comprometimento da chave secreta (KCI - Key Compromise Impersonation): se a chave secreta de longa duração de um participante $A$ for comprometida, um atacante não deve ser capaz de personificar um participante $B$ perante $A$.

- Segurança futura perfeita (PFS - Perfect Forward Secrecy): deve ser inviável para um atacante recuperar uma chave de sessão mesmo que, no futuro, venha a corromper as chaves secretas de longa duração de todos os participantes envolvidos naquela sessão. 
- Segurança futura perfeita-fraca (wPFS - Weak Perfect Forward Secrecy): semelhante à propriedade PFS, com a condição de que o adversário não esteja ativamente envolvido na escolha dos segredos temporários utilizados no cálculo da chave de sessão. Em Krawczyk (2005) foi demonstrado que em protocolos com apenas duas passagens de mensagens, wPFS é o melhor que se pode alcançar com relação à segurança no futuro.

- Resistência ao vazamento de segredos temporários: o vazamento de um valor secreto temporário não deve comprometer a segurança de sessões que não o tenham utilizado.

Para os casos em que não são necessários certificados digitais para autenticar as chaves públicas, são desejáveis duas propriedades adicionais:

- Segurança no futuro perante do KGC (KGC Forward Secrecy): o KGC não deve ser capaz de calcular as chaves de sessão, mesmo que monitore todo o tráfego durante o processo de estabelecimento das chaves e que, portanto, tenha acesso a todos os dados públicos.

- Resistência ao vazamento de segredos temporários para o KGC: em protocolos de acordo de chave com autenticação (AKA) no modelo sem certificado, o KGC deve ser incapaz de calcular a chave de sessão, mesmo estando de posse dos valores secretos temporários daquela sessão, e fazendo uso de seu conhecimento de todas as chaves secretas parciais.

\subsubsection{Modelos de segurança}

Mesmo que um protocolo seja resistente contra um subconjunto de ataques, não há garantias de que continue seguro caso um adversário tente combinar vários deles. Este problema fez com que os projetistas de protocolos de acordo de chave recorressem à segurança provável como uma ferramenta analítica (Swanson, 2008).

Essencialmente, o objetivo da segurança provável é demonstrar que um protocolo $\mathcal{P}$ é seguro sob a hipótese de um problema computacional ou uma primitiva $\mathcal{S}$. Para isso, leva-se em conta quais serão as capacidades e objetivos do atacante (modelo do adversário), com intuito de provar que o atacante deve ser capaz de resolver $\mathcal{S}$ a fim de quebrar a segurança do sistema modelado.

Em um modelo de segurança para protocolos AKA, o adversário, os participantes e as sessões entre os participantes são modelados com máquinas de Turing probabilísticas, denominadas oráculos. Todas as comunicações entre os participantes são controladas pelo adversário, que tem o poder de criar usuários, interagir com as sessões e iniciá-las como bem entender. Portanto, um modelo de segurança deve dispor de vários atributos de segurança, como os apresentados na Seção 4.4.1. Recomendamos a leitura de Swanson (2008) e Goya (2011) para definições formais de modelos de segurança e funcionamento dos oráculos.

A seguir faremos uma breve descrição dos principais modelos de segurança existentes. A diferença fundamental entre eles está no poder do adversário. Quanto maior seu poder, mais seguro é o modelo.

- Canetti-Krawczyk (CK): proposto por Canetti e Krawczyk (2001), apresenta uma análise para o uso de protocolos de acordo de chave combinados com sistemas de criptografia simétrica e funções de autenticação. Consequentemente, protocolos demonstrados seguros no modelo CK são utilizados para criar canais seguros, ou seja, fornecem um canal de transmissão de dados secreto e autenticado.

- Extended Canetti-Krawczyk (eCK): o modelo eCK, proposto por LaMacchia et al. (2007), assegura todas as propriedades de segurança do modelo CK, além de ser resistente à ataques KCI, atacantes internos, e vazamento de segredos temporários da sessão. Enquanto que no modelo CK todos os segredos temporários de uma sessão podem ser revelados de uma única vez, no eCK os temporários podem ser comprometidos um a um, à escolha do adversário.

O trabalho de Swanson (2008) mostrou que eram necessárias adaptações ao modelo eCK para o caso de protocolos no modelo sem certificado, tendo explorado algumas vulnerabilidades de protocolos considerados seguros pela literatura. Em Swanson e Jao (2009b) é proposta uma extensão sobre 
o modelo eCK, que chamaremos de SJ. A partir de então, surgiram outras melhorias ao modelo SJ, propostas por Lippold et al. (2009) e Goya (2011). Dentre estes novos modelos, nos interessam:

- LBG: modelo de Lippold et al. (2009). É uma extensão do modelo SJ, que propõe maiores poderes ao atacante. Dizemos que é um modelo contra adversário forte.

- SJ: modelo de Swanson e Jao (2009b). É mais fraco que o modelo LBG devido a restrições no oráculo que revela para o adversário a chave de uma determinada sessão. Neste modelo, o usuário continua a usar o seu próprio par original de chaves para calcular a chave de sessão, mesmo que o adversário tenha substituído a chave pública. No LBG, até mesmo o usuário dono da chave pública passaria a utilizar a nova chave pública escolhida pelo adversário. Outra diferença é que, no modelo de segurança SJ, o adversário que conhece a chave mestra secreta não pode substituir chaves públicas. Portanto, dizemos que este modelo é contra adversário moderado.

- LG: modelo de Lippold e Nieto (2010), com maiores restrições ao atacante. Dizemos que é um modelo contra adversário fraco.

- $\mathbf{S J}^{+}$: modelo de Goya (2011). Apresenta melhorias ao modelo SJ, contra adversário moderado.

- Mal-LBG: modelo de Goya (2011). Extensão do modelo LBG contra autoridade mal intencionada.

- Mal-SJ' ${ }^{+}$modelo de Goya (2011). Extensão do modelo $\mathrm{SJ}^{+}$contra autoridade mal intencionada.

Estes modelos podem ser relacionados entre si, de acordo com a representação da Figura 4.4. Podemos afirmar que o modelo LG é o mais fraco de todos, seguido pelos modelos $\mathrm{SJ} \mathrm{SJ}^{+}, \mathrm{LBG}$, e Mal-SJ+, enquanto o modelo Mal-LBG é o mais forte.

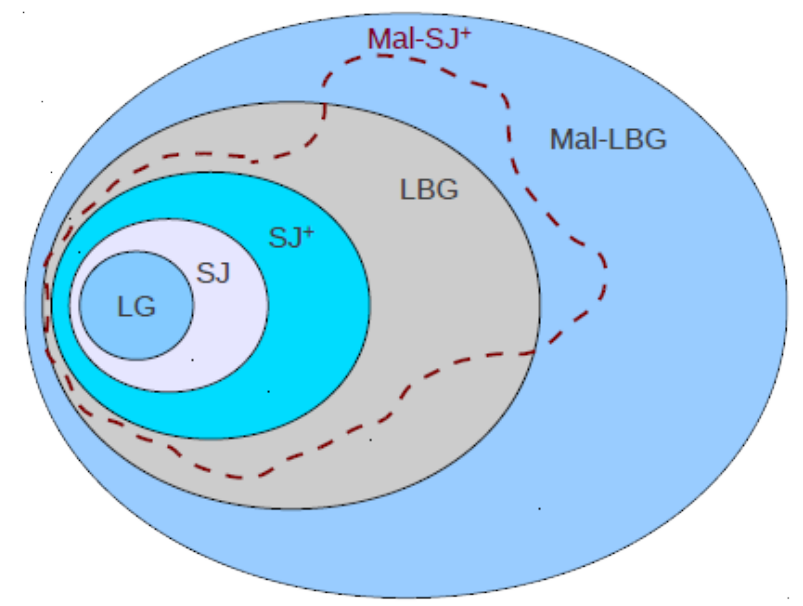

Figura 4.4: Hierarquia dos modelos de segurança para protocolos no modelo sem certificado (Goya, 2011). 


\section{Capítulo 5}

\section{Protocolos Estudados}

O objetivo deste capítulo é apresentar, em detalhes, os protocolos de acordo de chave estudados para realização dos experimentos que serão apresentados no Capítulo 6.

\subsection{Escolha dos protocolos}

O critério de escolha dos protocolos analisados levou em consideração aspectos como o modelo de segurança empregado, relevância do protocolo (quantidade de citações aos artigos em que são apresentados), ano de publicação (preferência por protocolos apresentados recentemente) e ausência de publicações científicas que comprovem falhas de segurança ou quebra do protocolo.

Os protocolos selecionados no modelo baseado em identidade foram demonstrados seguros sob os modelos de segurança CK ou eCK. Já os protocolos escolhidos no modelo sem certificado foram demonstrados seguros sob os modelos de segurança LBG, $\mathrm{SJ}^{+}$, Mal-LBG e Mal-SJ ${ }^{+}$, todos melhorias do modelo eCK para o caso sem certificado, e considerados seguros contra adversários forte, moderado, e autoridade mal intencionada.

As próximas subseções detalham cada um dos protocolos escolhidos. Um diagrama representa os cálculos realizados e mensagens trocadas pelos usuários $A$ e $B$. Na sequência do diagrama é apresentada a corretude do protocolo, demonstrando que a chave de sessão calculada por $A$ (chamada $S K_{A}$ ) é igual ao valor da chave de sessão calculada por $B$ (chamada $S K_{B}$ ). Com o propósito de simplificar os cálculos, foi adotada a notação simétrica, isto é, empregando-se emparelhamentos na forma $e: \mathbb{G} \times \mathbb{G} \rightarrow \mathbb{G}_{T}$. Tal notação foi escolhida por sua simplicidade, uma vez que não é necessário separar quais elementos pertenceriam ao grupo $\mathbb{G}_{1}$ e quais pertenceriam ao grupo $\mathbb{G}_{2}$ (já que $\mathbb{G}_{1}=\mathbb{G}_{2}$ ), facilitando a compreensão dos diagramas apresentados.

\subsection{Protocolos no modelo baseado em identidade}

Protocolos de acordo de chave no modelo baseado em identidade podem ser subdivididos em quatro etapas principais: inicialização do sistema, geração das chaves dos usuários, troca de mensagens e cálculo da chave compartilhada. A Figura 5.1 sintetiza cada uma destas etapas. 


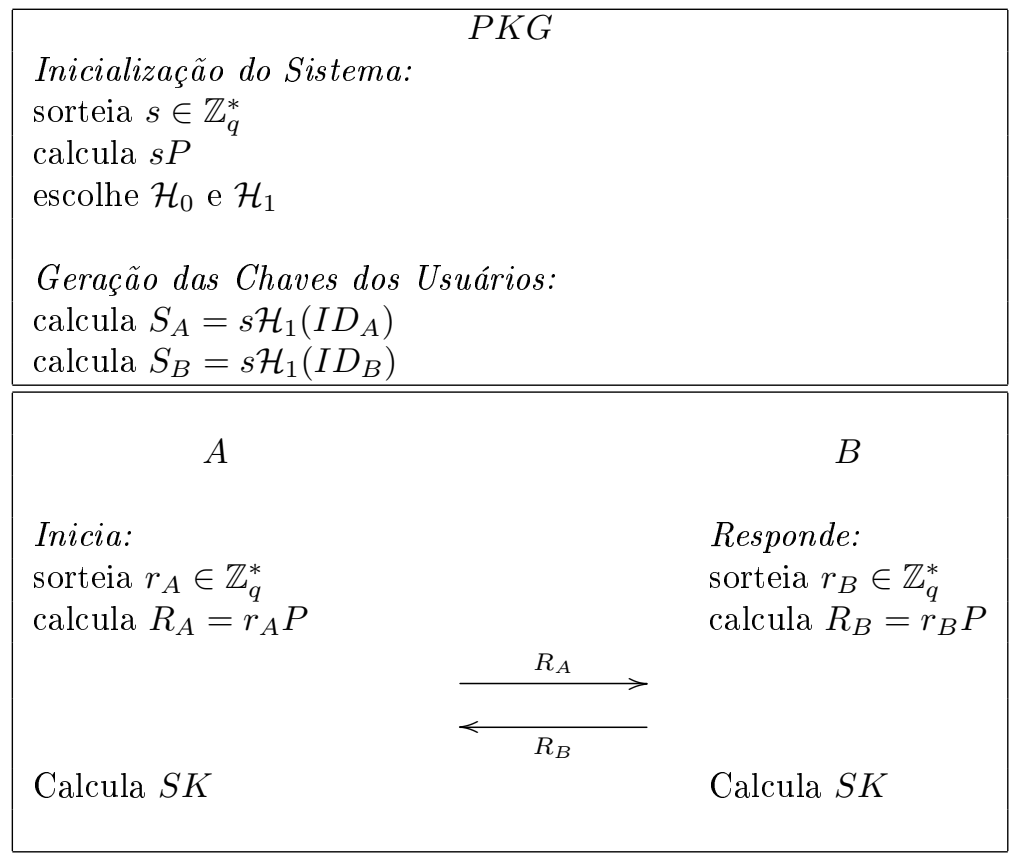

Figura 5.1: Representação genérica de um protocolo de acordo de chave no modelo baseado em identidades

A seguir estenderemos as noções apresentadas na Seção 4.1 e detalharemos cada etapa.

\section{Inicialização do Sistema}

Dado um parâmetro de segurança $k$, a autoridade do sistema (PKG) seleciona dois grupos (elípticos) cíclicos: $\mathbb{G}$ aditivo e $\mathbb{G}_{T}$ multiplicativo, ambos de ordem $q$. Seja $P \in \mathbb{G}$ um gerador de $\mathbb{G}$ e seja $e: \mathbb{G} \times \mathbb{G} \rightarrow \mathbb{G}_{T}$ um emparelhamento bilinear admissível.

O PKG escolhe $s \stackrel{\$}{\leftarrow} \mathbb{Z}_{q}^{*}$, que será a chave mestra secreta do sistema, e calcula sua chave pública $s P$. Ele também seleciona as funções de espalhamento ${ }^{1} \mathcal{H}_{0}, \mathcal{H}_{1}$, tais que $\mathcal{H}_{0}:\{0,1\}^{*} \rightarrow\{0,1\}^{k}$ e $\mathcal{H}_{1}:\{0,1\}^{*} \rightarrow \mathbb{G}$. Por fim, a autoridade do sistema torna públicos os parâmetros params $=\left\langle k, \mathbb{G}, \mathbb{G}_{T}, q, e, P, s P, \mathcal{H}_{0}, \mathcal{H}_{1}\right\rangle$.

Alguns dos protocolos estudados neste trabalho necessitam de uma versão estendida dos parâmetros do PKG. Para estes casos, consideraremos todos os parâmetros contidos em params, com adição de uma nova função de espalhamento $\mathcal{H}_{2}$, tal que $\mathcal{H}_{2}:\{0,1\}^{*} \rightarrow \mathbb{G}$. Neste caso, o PKG divulga os parâmetros públicos params_ext $=\left\langle k, \mathbb{G}, \mathbb{G}_{T}, q, e, P, s P, \mathcal{H}_{0}, \mathcal{H}_{1}, \mathcal{H}_{2}\right\rangle$.

\section{Geração das Chaves dos Usuários}

Seja $I D_{U} \in\{0,1\}^{*}$ a identidade de um usuário $U$, e seja o valor público $Q_{U_{1}}=\mathcal{H}_{1}\left(I D_{U}\right) \in \mathbb{G}$. Para cada usuário $U$, o PKG calcula a chave secreta de $U$ como sendo $S_{U_{1}}=s Q_{U_{1}} \in \mathbb{G}$.

Nos protocolos que utilizam os parâmetros estendidos, o PKG também deve calcular $S_{U_{2}}=$ $s Q_{U_{2}} \in \mathbb{G}$, a partir do valor público $Q_{U_{2}}=\mathcal{H}_{2}\left(I D_{U}\right) \in \mathbb{G}$. Nestes casos, a chave secreta do usuário $U$ é o $\operatorname{par}\left\langle S_{U_{1}}, S_{U_{2}}\right\rangle$.

Ao fim deste processo, o PKG envia $S_{U_{1}}$ e $S_{U_{2}}$ (quando utilizada) para $U$, através de um canal seguro e autenticado.

\section{Troca de Mensagens}

Considere dois usuários $A$ e $B$ que desejem estabelecer uma chave secreta em comum. O participante $A$ sorteia um valor secreto temporário $r_{A} \stackrel{\$}{\leftarrow} \mathbb{Z}_{q}^{*}$, e calcula $R_{A}=r_{A} P$. De maneira análoga,

\footnotetext{
${ }^{1}$ Funções de hash criptográficas.
} 
$B$ sorteia um valor secreto temporário $r_{B} \stackrel{\$}{\leftarrow} \mathbb{Z}_{q}^{*}$ e calcula $R_{B}=r_{B} P$. O usuário $A$, então, envia $R_{A}$ para o usuário $B$, que por sua vez envia $R_{B}$ para o usuário $A$.

Estes valores são importantes pois garantem que os participantes de um protocolo de acordo de chave estabeleçam uma chave compartilhada diferente à cada nova rodada. Desta forma, a chave gerada na i-ésima rodada do protocolo será diferente da chave gerada na j-ésima rodada (sendo $i \neq j)$.

\section{Cálculo da Chave Compartilhada}

Após a execução das etapas anteriores, e em posse dos parâmetros públicos e das mensagens recebidas, os participantes do protocolo calculam a chave compartilhada. Nesta etapa, os usuários $A$ e $B$ calculam valores temporários que serão concatenados e em seguida utilizados em uma função de hash segura $\left(\mathcal{H}_{0}\right)$ para se chegar à chave compartilhada.

\subsubsection{Protocolo Huang-Cao}

O protocolo de Huang e Cao (2009), representado na Figura 5.2, foi demonstrado seguro no modelo de segurança eCK, sob a hipótese do problema BDH.

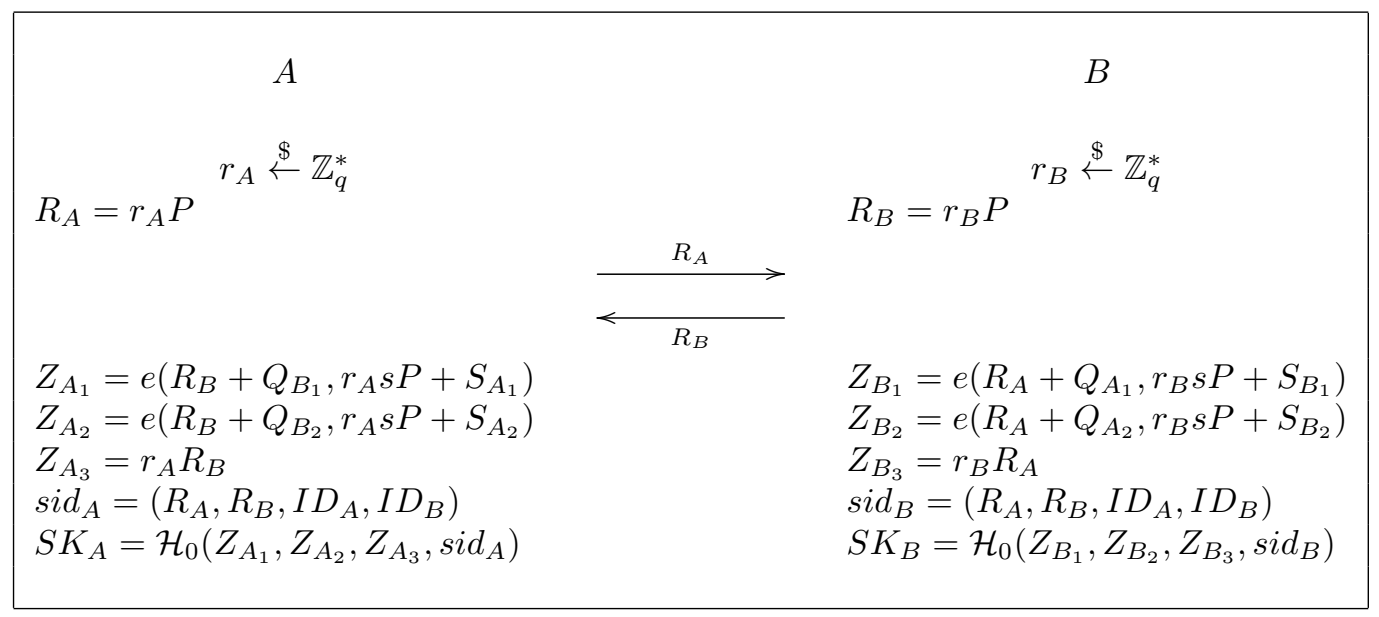

Figura 5.2: Protocolo $H C$, seguro sob o problema $B D H$.

\section{Corretude}

$$
\begin{gathered}
Z_{A_{1}}=e\left(R_{B}+Q_{B_{1}}, r_{A} s P+S_{A_{1}}\right)=e\left(R_{B}+Q_{B_{1}}, r_{A} s P+s Q_{A_{1}}\right)=e\left(r_{B} P+Q_{B_{1}}, r_{A} s P+s Q_{A_{1}}\right)= \\
e\left(r_{B} P+Q_{B_{1}}, r_{A} P+Q_{A_{1}}\right)^{s}=e\left(r_{B} s P+s Q_{B_{1}}, r_{A} P+Q_{A_{1}}\right)=e\left(r_{B} s P+S_{B_{1}}, R_{A}+Q_{A_{1}}\right)=Z_{B_{1}} \\
Z_{A_{2}}=e\left(R_{B}+Q_{B_{2}}, r_{A} s P+S_{A_{2}}\right)=e\left(R_{B}+Q_{B_{2}}, r_{A} s P+s Q_{A_{2}}\right)=e\left(r_{B} P+Q_{B_{2}}, r_{A} s P+s Q_{A_{2}}\right)= \\
e\left(r_{B} P+Q_{B_{2}}, r_{A} P+Q_{A_{2}}\right)^{s}=e\left(r_{B} s P+s Q_{B_{2}}, r_{A} P+Q_{A_{2}}\right)=e\left(r_{B} s P+S_{B_{2}}, R_{A}+Q_{A_{2}}\right)=Z_{B_{2}} \\
Z_{A_{3}}=r_{A} R_{B}=r_{A} r_{B} P=r_{B} R_{A}=Z_{B_{3}}
\end{gathered}
$$

\subsubsection{Protocolo Hu-Liu-Zhang}

O protocolo de Hu et al. (2009), representado na Figura 5.3, foi demonstrado seguro no modelo de segurança eCK, sob a hipótese do problema Gap-BDH. 


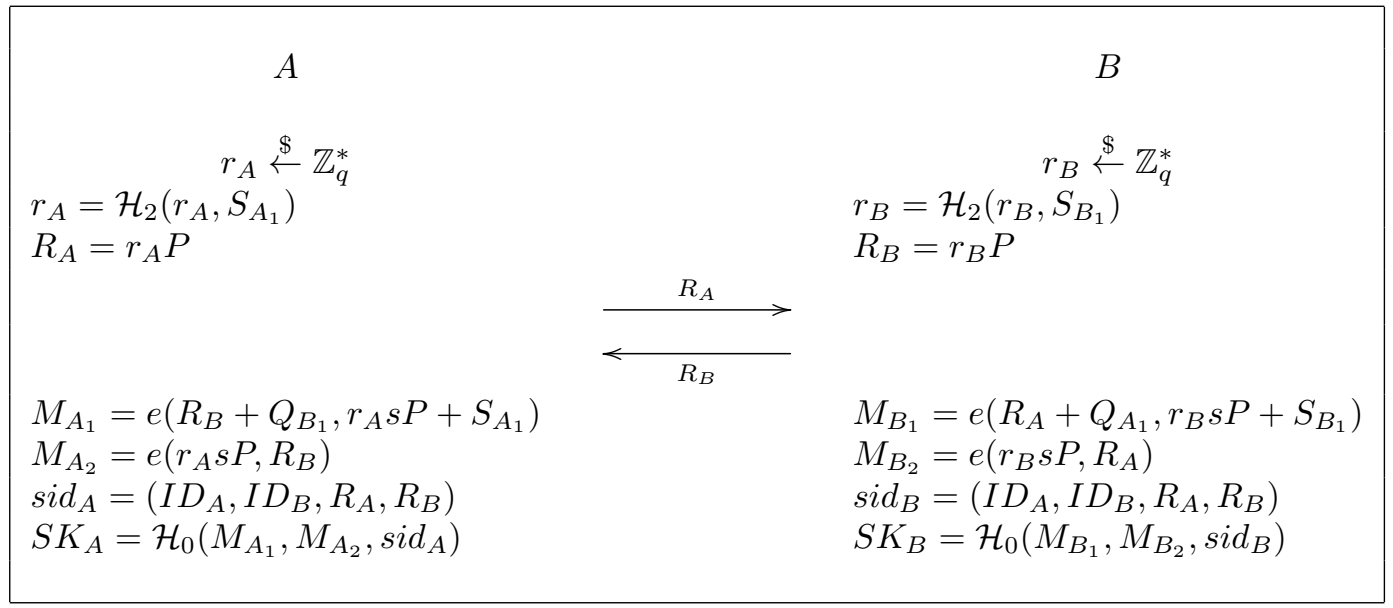

Figura 5.3: Protocolo HLZ, seguro sob o problema Gap-BDH.

\section{Corretude}

$$
\begin{gathered}
M_{A_{1}}=e\left(R_{B}+Q_{B_{1}}, r_{A} s P+S_{A_{1}}\right)=e\left(R_{B}+Q_{B_{1}}, r_{A} s P+s Q_{A_{1}}\right)=e\left(r_{B} P+Q_{B_{1}}, r_{A} s P+s Q_{A_{1}}\right)= \\
e\left(r_{B} P+Q_{B_{1}}, r_{A} P+Q_{A_{1}}\right)^{s}=e\left(r_{B} s P+s Q_{B_{1}}, r_{A} P+Q_{A_{1}}\right)=e\left(r_{B} s P+S_{B_{1}}, R_{A}+Q_{A_{1}}\right)=M_{B_{1}} \\
M_{A_{2}}=e\left(r_{A} s P, R_{B}\right)=e\left(r_{A} P, r_{B} P\right)^{s}=e\left(r_{A} P, r_{B} s P\right)=e\left(R_{A}, r_{B} s P\right)=M_{B_{2}}
\end{gathered}
$$

\subsubsection{Protocolo Chow-Choo}

O protocolo de Chow e Choo (2007), representado na Figura 5.4, foi demonstrado seguro no modelo de segurança CK, sob a hipótese do problema BDH.

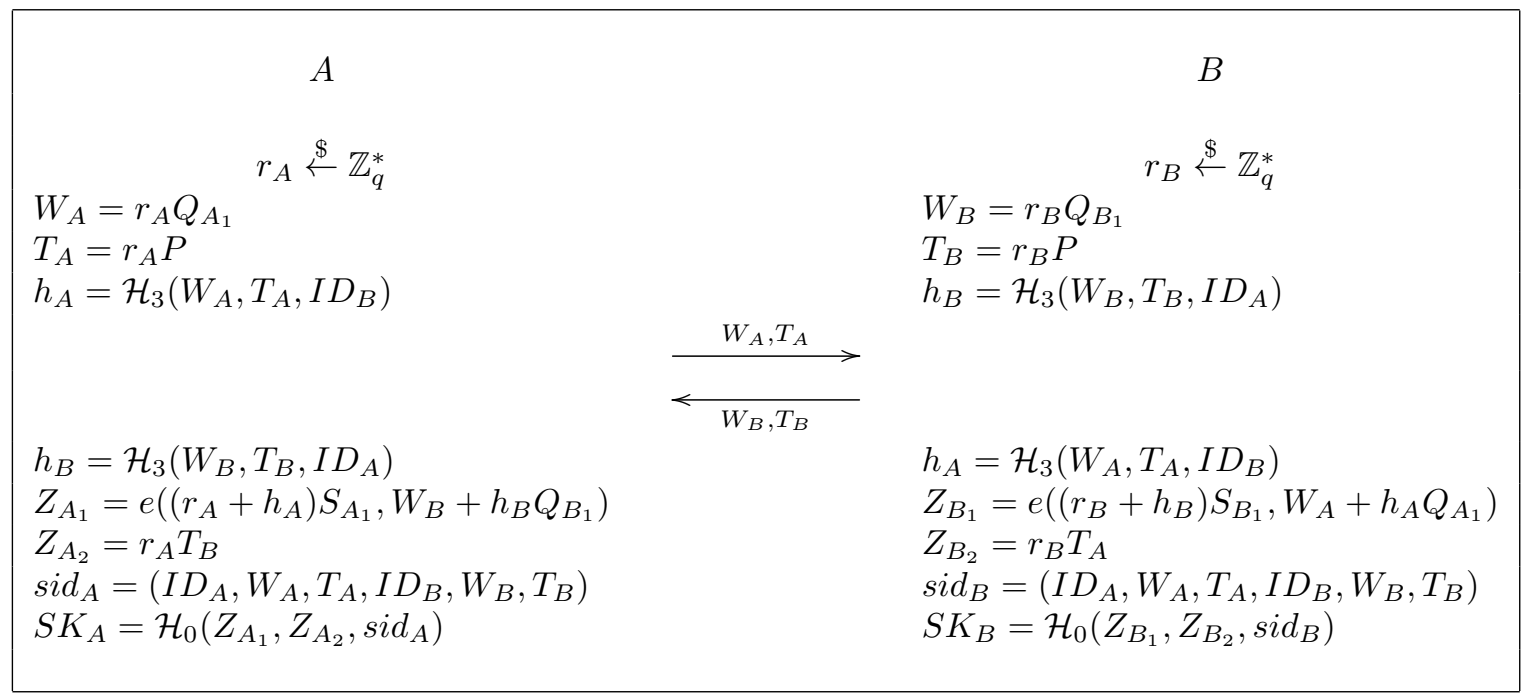

Figura 5.4: Protocolo CC, seguro sob o problema $B D H$.

\section{Corretude}

$Z_{A_{1}}=e\left(\left(r_{A}+h_{A}\right) S_{A_{1}}, W_{B}+h_{B} Q_{B_{1}}\right)=e\left(\left(r_{A}+h_{A}\right) S_{A_{1}}, r_{B} Q_{B_{1}}+h_{B} Q_{B_{1}}\right)=e\left(\left(r_{A}+h_{A}\right) s Q_{A_{1}},\left(r_{B}+\right.\right.$ $\left.\left.h_{B}\right) Q_{B_{1}}\right)=e\left(\left(r_{A}+h_{A}\right) Q_{A_{1}},\left(r_{B}+h_{B}\right) Q_{B_{1}}\right)^{s}=e\left(\left(r_{A}+h_{A}\right) Q_{A_{1}},\left(r_{B}+h_{B}\right) s Q_{B_{1}}\right)=e\left(\left(r_{A}+\right.\right.$ $\left.\left.h_{A}\right) Q_{A_{1}},\left(r_{B}+h_{B}\right) S_{B_{1}}\right)=e\left(\left(r_{B}+h_{B}\right) S_{B_{1}}, W_{A}+h_{A} Q_{A_{1}}\right)=Z_{B_{1}}$

$$
Z_{A_{2}}=r_{A} T_{B}=r_{A} r_{B} P=r_{B} T_{A}=Z_{B_{2}}
$$




\subsubsection{Protocolo Ni-Chen-Li-Hao}

O trabalho de Ni et al. (2011) propôs dois protocolos, representados nas Figuras 5.5 e 5.6. Foram demonstrados seguros no modelo de segurança eCK, sob as hipóteses dos problemas BDH e Gap-BDH, respectivamente.

Ambos os protocolos possuem uma propriedade conhecida como modo escrow. Isto significa que o PKG pode recuperar todas as chaves de sessão calculadas pelos participantes, utilizando sua chave privada $s$ e outros valores públicos, como $R_{A}$ e $R_{B}$. Portanto, tais protocolos são um caso especial de acordo de chave, e são recomendados apenas em cenários muito específicos. Por exemplo, quando houver necessidade do PKG fazer uma auditoria das mensagens secretas trocadas pelos usuários, calculando a chave secreta acordada entre eles.

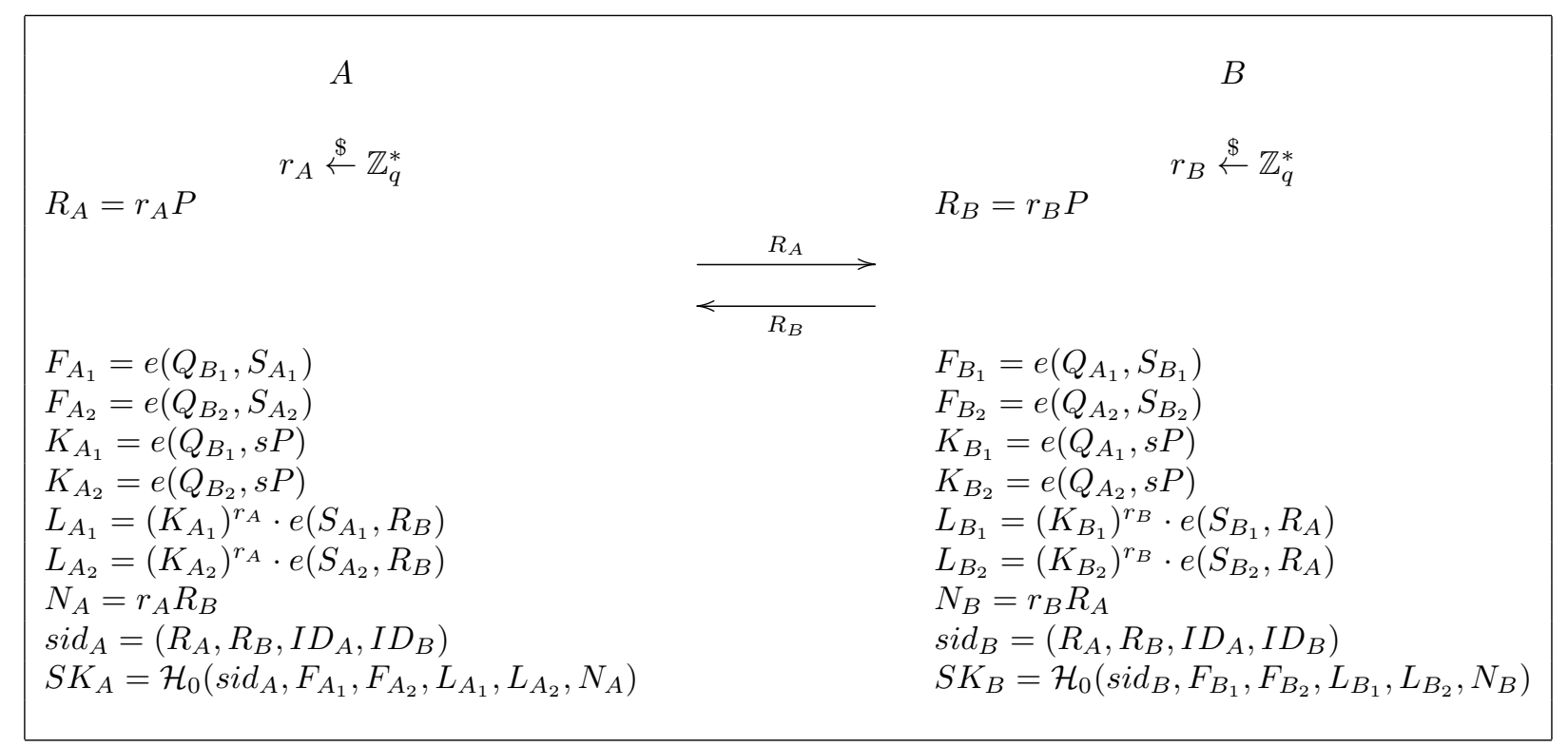

Figura 5.5: Protocolo NLCH, seguro sob o problema BDH.

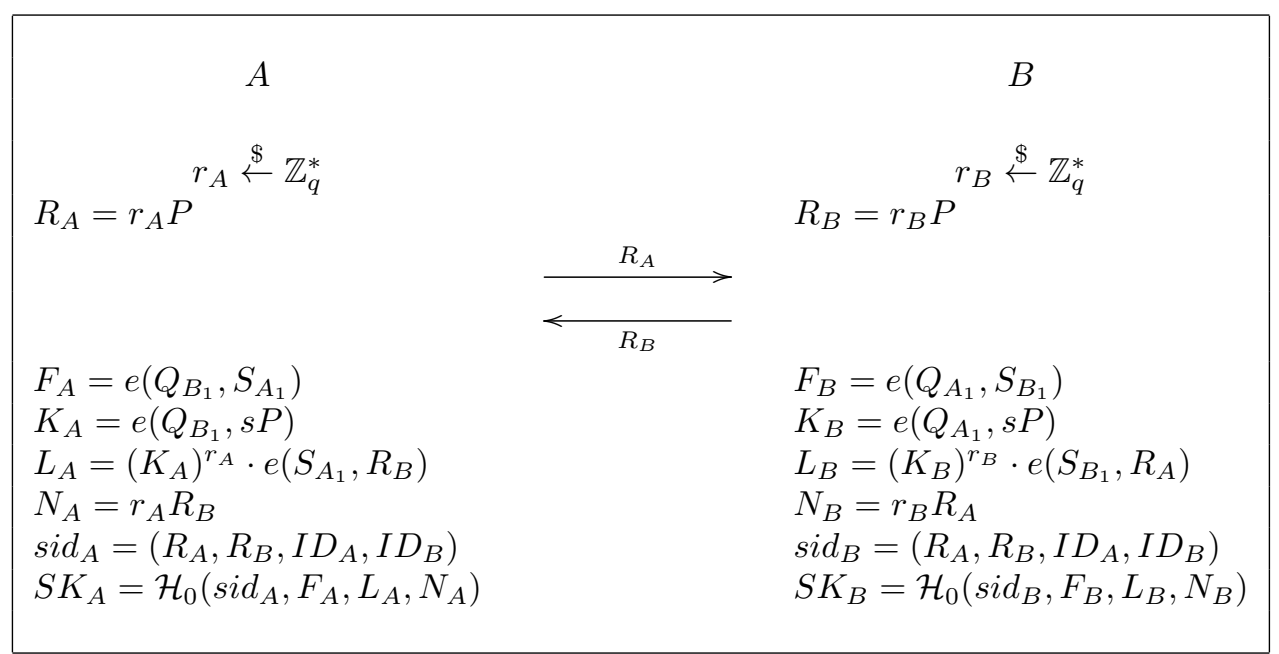

Figura 5.6: Protocolo NLCH, seguro sob o problema Gap-BDH.

\section{Corretude}

$$
\begin{aligned}
& F_{A_{1}}=e\left(Q_{B_{1}}, S_{A_{1}}\right)=e\left(Q_{B_{1}}, s Q_{A_{1}}\right)=e\left(Q_{B_{1}}, Q_{A_{1}}\right)^{s}=e\left(s Q_{B_{1}}, Q_{A_{1}}\right)=e\left(S_{B_{1}}, Q_{A_{1}}\right)=F_{B_{1}} \\
& F_{A_{2}}=e\left(Q_{B_{2}}, S_{A_{2}}\right)=e\left(Q_{B_{2}}, s Q_{A_{2}}\right)=e\left(Q_{B_{2}}, Q_{A_{2}}\right)^{s}=e\left(s Q_{B_{2}}, Q_{A_{2}}\right)=e\left(S_{B_{2}}, Q_{A_{2}}\right)=F_{B_{2}}
\end{aligned}
$$


$L_{A_{1}}=\left(K_{A_{1}}\right)^{r_{A}} \cdot e\left(S_{A_{1}}, R_{B}\right)=e\left(Q_{B_{1}}, s P\right)^{r_{A}} \cdot e\left(S_{A_{1}}, R_{B}\right)=e\left(Q_{B_{1}}, r_{A} P\right)^{s} \cdot e\left(S_{A_{1}}, R_{B}\right)$

$=e\left(s Q_{B_{1}}, r_{A} P\right) \cdot e\left(S_{A_{1}}, R_{B}\right)=e\left(S_{B_{1}}, R_{A}\right) \cdot e\left(s Q_{A_{1}}, r_{B} P\right)=e\left(S_{B_{1}}, R_{A}\right) \cdot e\left(Q_{A_{1}}, r_{B} P\right)^{s}$

$=e\left(S_{B_{1}}, R_{A}\right) \cdot e\left(Q_{A_{1}}, s P\right)^{r_{B}}=\left(K_{B_{1}}\right)^{r_{B}} \cdot e\left(S_{B_{1}}, R_{A}\right)=L_{B_{1}}$

$L_{A_{2}}=\left(K_{A_{2}}\right)^{r_{A}} \cdot e\left(S_{A_{2}}, R_{B}\right)=e\left(Q_{B_{2}}, s P\right)^{r_{A}} \cdot e\left(S_{A_{2}}, R_{B}\right)=e\left(Q_{B_{2}}, r_{A} P\right)^{s} \cdot e\left(S_{A_{2}}, R_{B}\right)$

$=e\left(s Q_{B_{2}}, r_{A} P\right) \cdot e\left(S_{A_{2}}, R_{B}\right)=e\left(S_{B_{2}}, R_{A}\right) \cdot e\left(s Q_{A_{2}}, r_{B} P\right)=e\left(S_{B_{2}}, R_{A}\right) \cdot e\left(Q_{A_{2}}, r_{B} P\right)^{s}$

$=e\left(S_{B_{2}}, R_{A}\right) \cdot e\left(Q_{A_{2}}, s P\right)^{r_{B}}=\left(K_{B_{2}}\right)^{r_{B}} \cdot e\left(S_{B_{2}}, R_{A}\right)=L_{B_{2}}$

$N_{A}=r_{A} R_{B}=r_{A} r_{B} P=r_{B} R_{A}=N_{B}$

\section{Modo escrow}

$$
\begin{aligned}
& F_{A_{1}}=F_{B_{1}}=e\left(Q_{B_{1}}, S_{A_{1}}\right)=e\left(Q_{B_{1}}, Q_{A_{1}}\right)^{s} \\
& F_{A_{2}}=F_{B_{2}}=e\left(Q_{B_{2}}, S_{A_{2}}\right)=e\left(Q_{B_{2}}, Q_{A_{2}}\right)^{s} \\
& L_{A_{1}}=L_{B_{1}}=\left(K_{A_{1}}\right)^{r_{A}} \cdot e\left(S_{A_{1}}, R_{B}\right)=e\left(Q_{B_{1}}, s P\right)^{r_{A}} \cdot e\left(S_{A_{1}}, R_{B}\right)=e\left(Q_{B_{1}}, P\right)^{r_{A} s} \cdot e\left(Q_{A_{1}}, R_{B}\right)^{s}= \\
& e\left(Q_{B_{1}}, R_{A}\right)^{s} \cdot e\left(Q_{A_{1}}, R_{B}\right)^{s}
\end{aligned}
$$

$L_{A_{2}}=L_{B_{2}}=\left(K_{A_{2}}\right)^{r_{A}} \cdot e\left(S_{A_{2}}, R_{B}\right)=e\left(Q_{B_{2}}, s P\right)^{r_{A}} \cdot e\left(S_{A_{2}}, R_{B}\right)=e\left(Q_{B_{2}}, P\right)^{r_{A} s} \cdot e\left(Q_{A_{2}}, R_{B}\right)^{s}=$ $e\left(Q_{B_{2}}, R_{A}\right)^{s} \cdot e\left(Q_{A_{2}}, R_{B}\right)^{s}$

$$
N_{A}=N_{B}=e(P, P)^{r_{A} r_{B} s}=e\left(R_{A}, R_{B}\right)^{s}
$$

\subsubsection{Protocolo Ni-Chen-Li}

O protocolo de Ni et al. (2012), representado na Figura 5.4, foi demonstrado seguro no modelo de segurança eCK, sob a hipótese do problema BDH. Assim como o protocolo de Ni et al. (2011), também possui modo escrow.

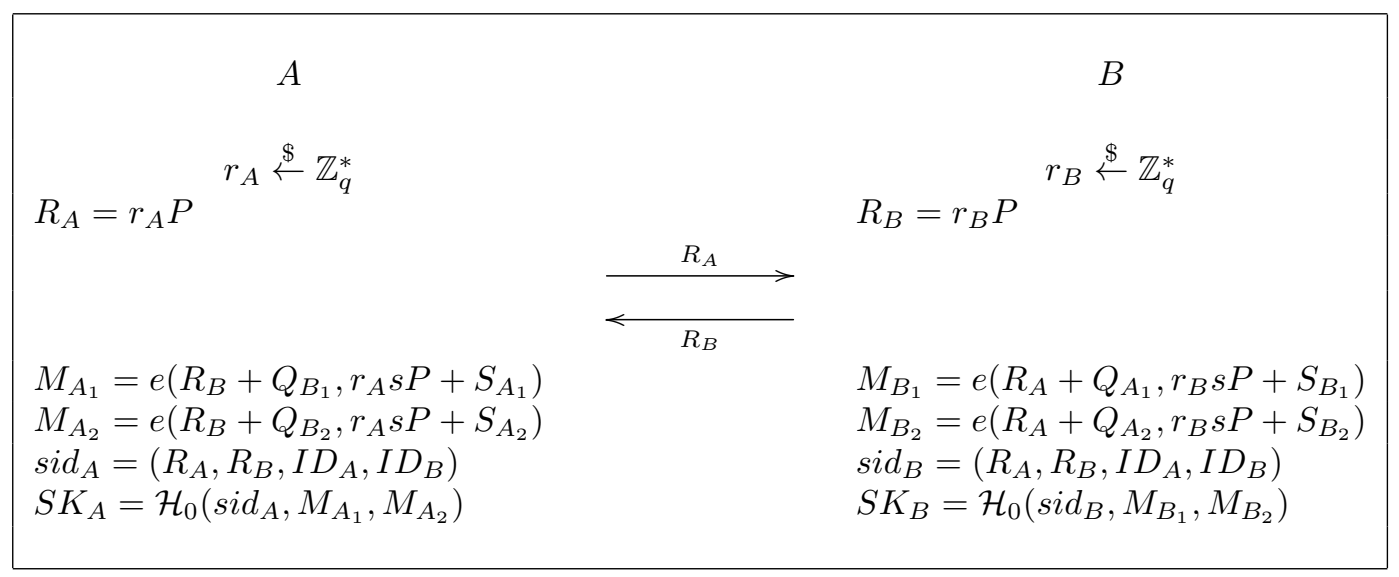

Figura 5.7: Protocolo $N L C$, seguro sob o problema $B D H$.

\section{Corretude}

$$
\begin{gathered}
M_{A_{1}}=e\left(R_{B}+Q_{B_{1}}, r_{A} s P+S_{A_{1}}\right)=e\left(r_{B} P+Q_{B_{1}}, r_{A} s P+s Q_{A_{1}}\right)=e\left(r_{B} P+Q_{B_{1}}, r_{A} P+Q_{A_{1}}\right)^{s}= \\
e\left(r_{B} s P+s Q_{B_{1}}, r_{A} P+Q_{A_{1}}\right)=e\left(r_{B} s P+S_{B_{1}}, R_{A}+Q_{A_{1}}\right)=M_{B_{1}} \\
M_{A_{2}}=e\left(R_{B}+Q_{B_{2}}, r_{A} s P+S_{A_{2}}\right)=e\left(r_{B} P+Q_{B_{2}}, r_{A} s P+s Q_{A_{2}}\right)=e\left(r_{B} P+Q_{B_{2}}, r_{A} P+Q_{A_{2}}\right)^{s}= \\
e\left(r_{B} s P+s Q_{B_{2}}, r_{A} P+Q_{A_{2}}\right)=e\left(r_{B} s P+S_{B_{2}}, R_{A}+Q_{A_{2}}\right)=M_{B_{2}}
\end{gathered}
$$




\section{Modo escrow}

$$
\begin{aligned}
& M_{A_{1}}=M_{B_{1}}=e\left(R_{B}+Q_{B_{1}}, r_{A} s P+S_{A_{1}}\right)=e\left(R_{B}+Q_{B_{1}}, r_{A} P+Q_{A_{1}}\right)^{s}=e\left(R_{B}+Q_{B_{1}}, R_{A}+Q_{A_{1}}\right)^{s} \\
& M_{A_{2}}=M_{B_{2}}=e\left(R_{B}+Q_{B_{2}}, r_{A} s P+S_{A_{2}}\right)=e\left(R_{B}+Q_{B_{2}}, r_{A} P+Q_{A_{2}}\right)^{s}=e\left(R_{B}+Q_{B_{2}}, R_{A}+Q_{A_{2}}\right)^{s}
\end{aligned}
$$

\subsection{Protocolos no modelo sem certificado}

Assim como no modelo baseado em identidade, os protocolos de acordo de chave no modelo sem certificado também são divididos em quatro etapas principais. As etapas de inicialização do sistema e de cálculo da chave compartilhada são idênticas às do modelo anterior. Já as etapas de geração de chaves dos usuários e troca de mensagens apresentam algumas diferenças, que veremos a seguir.

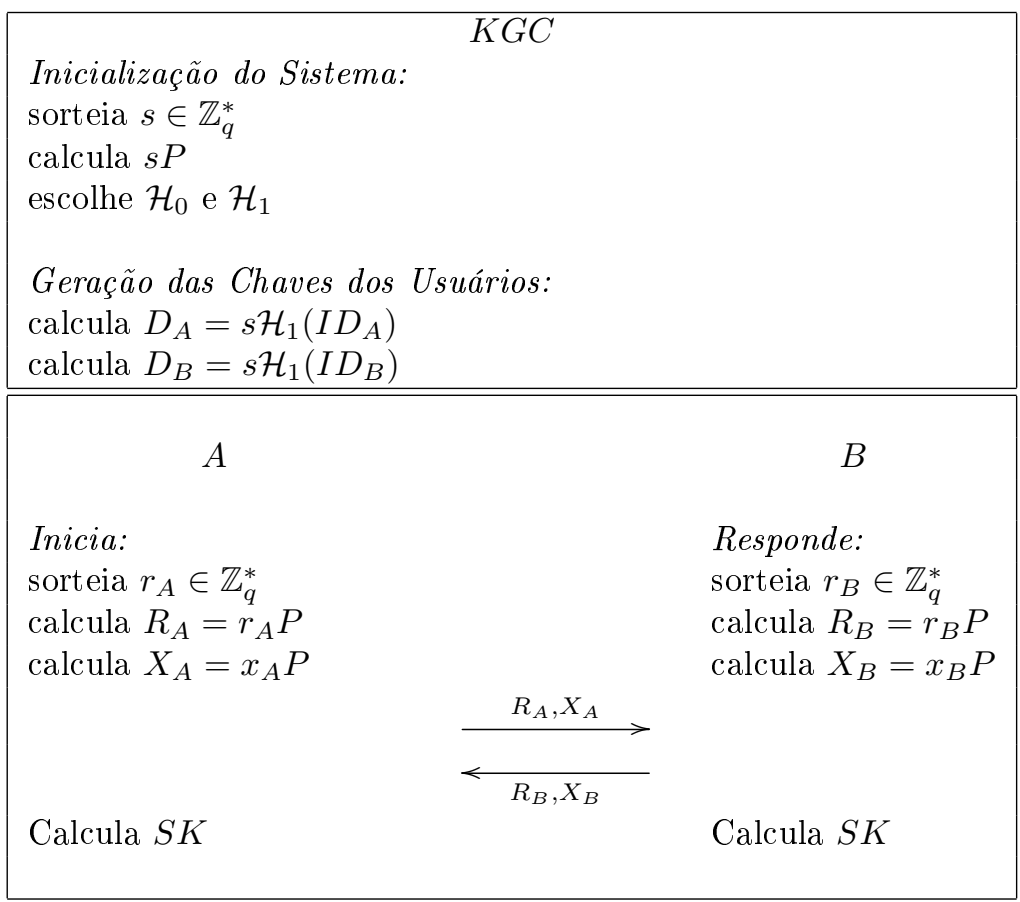

Figura 5.8: Representação genérica de um protocolo de acordo de chaves no modelo sem certificado

\section{Geração das Chaves dos Usuários}

Seja $I D_{U} \in\{0,1\}^{*}$ a identidade de um usuário $U$, e seja o valor público $Q_{U_{1}}=\mathcal{H}_{1}\left(I D_{U}\right) \in \mathbb{G}$. Para cada usuário $U$, o KGC calcula a chave secreta parcial de $U$ como sendo $D_{U_{1}}=s Q_{U_{1}} \in \mathbb{G}$.

Nos protocolos que utilizam os parâmetros estendidos, o KGC também deve calcular $D_{U_{2}}=$ $s Q_{U_{2}} \in \mathbb{G}$, a partir do valor público $Q_{U_{2}}=\mathcal{H}_{2}\left(I D_{U}\right) \in \mathbb{G}$. Nestes casos, a chave secreta parcial do usuário $U$ é o par $\left\langle D_{U_{1}}, D_{U_{2}}\right\rangle$.

O KGC então envia $D_{U_{1}}$ e $D_{U_{2}}$ (quando utilizada) para $U$, através de um canal seguro e autenticado.

Como visto na Seção 4.2, ao receber sua chave secreta parcial, $U$ escolhe um valor secreto $x_{U} \stackrel{\$}{\leftarrow} \mathbb{Z}_{q}^{*}$ e calcula sua chave secreta completa $S_{U_{1}}=x_{U} D_{U_{1}}$ (e $S_{U_{2}}=x_{U} D_{U_{2}}$ para protocolos que utilizam parâmetros estendidos). A partir de $x_{U}$ ele também calcula sua chave pública como sendo $x_{U} P$. 


\section{Troca de Mensagens}

Semelhante à troca de mensagens dos protocolos no modelo baseado em identidade, o usuário $A$ que deseja se comunicar com $B$ escolhe o valor secreto temporário $r_{A} \stackrel{\$}{\leftarrow} \mathbb{Z}_{q}^{*}$ e calcula $R_{A}=r_{A} P$. Da mesma forma, o usuário $B$ também escolhe um valor secreto temporário $r_{B} \stackrel{\$}{\leftarrow} \mathbb{Z}_{q}^{*}$ e calcula $R_{B}=r_{B} P$.

Além dos valores temporários, incluímos nas mensagens as chaves públicas dos usuários. Este não é um procedimento obrigatório caso seja garantida a entrega da chave pública por outro meio (através de um diretório de chaves públicas, por exemplo). No entanto, seguimos as recomendações de (Goya, 2011), que considera mais fácil um adversário alterar um diretório público do que modificar ativamente o tráfego de mensagens entre dois usuários. Para tal, o usuário $A$ escolhe um valor secreto $x_{A} \stackrel{\$}{\leftarrow} \mathbb{Z}_{q}^{*}$ e calcula sua chave pública $X_{A}=x_{A} P$. Analogamente, $B$ escolhe um valor secreto $x_{B} \stackrel{\$}{\leftarrow} \mathbb{Z}_{q}^{*}$ e calcula sua chave pública $X_{B}=x_{B} P$.

Ao final desta etapa, o usuário $A$ envia o par $\left\langle R_{A}, X_{A}\right\rangle$ para $B$, que por sua vez envia o par $\left\langle R_{B}, X_{B}\right\rangle$ para $A$.

\subsubsection{Protocolo Lippold-Boyd-González Nieto}

O trabalho de Lippold et al. (2009), como já discutido na Seção 4.4.2, propôs um modelo de segurança contra adversário forte ${ }^{2}$ para protocolos AKA sem certificado. Na mesma publicação, foram propostos dois protocolos, representados nas Figuras 5.9 e 5.10, seguros no modelo de segurança exposto, sob a hipótese dos problemas BDH e Gap-BDH, respectivamente.

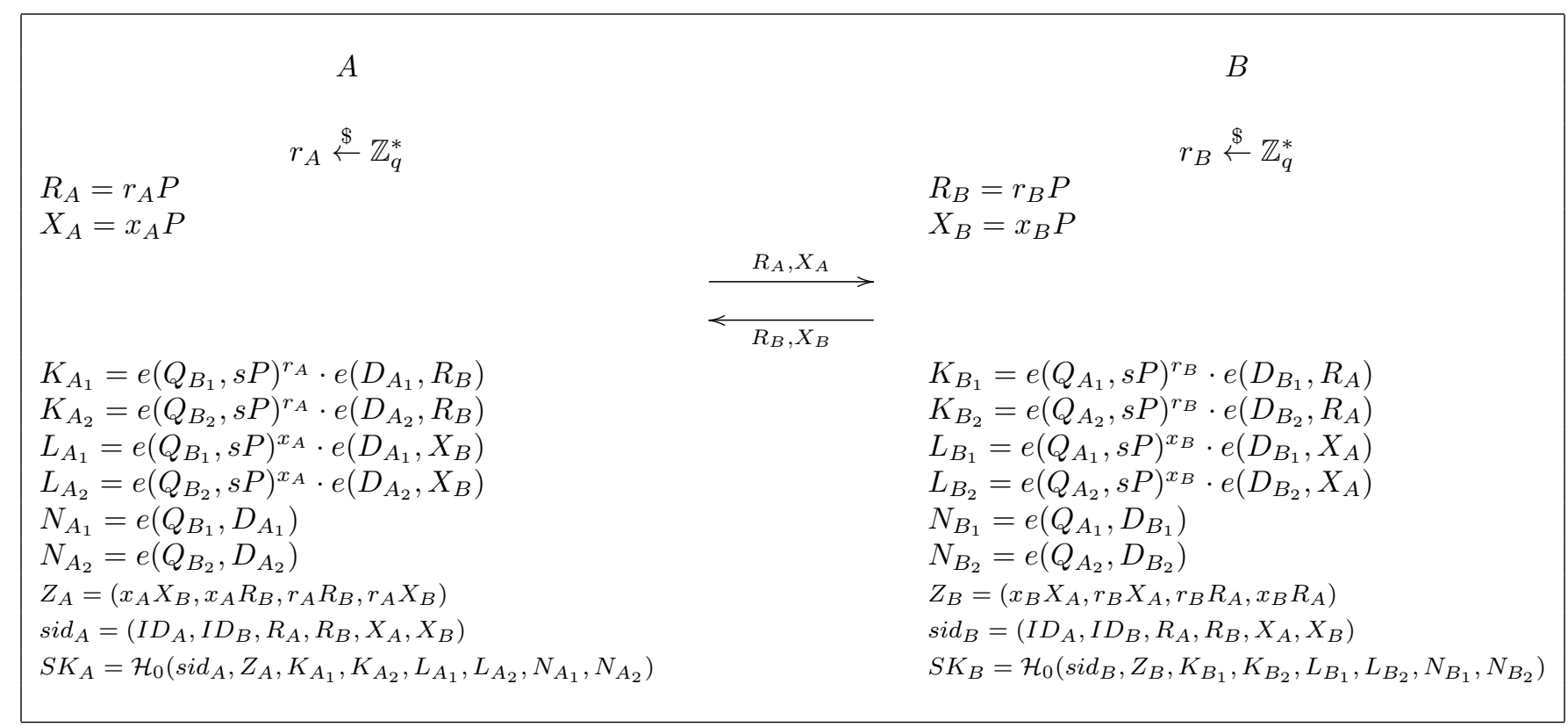

Figura 5.9: Protocolo $L B G$, seguro sob o problema $B D H$.

\footnotetext{
${ }^{2}$ Modelo de segurança LBG.
} 


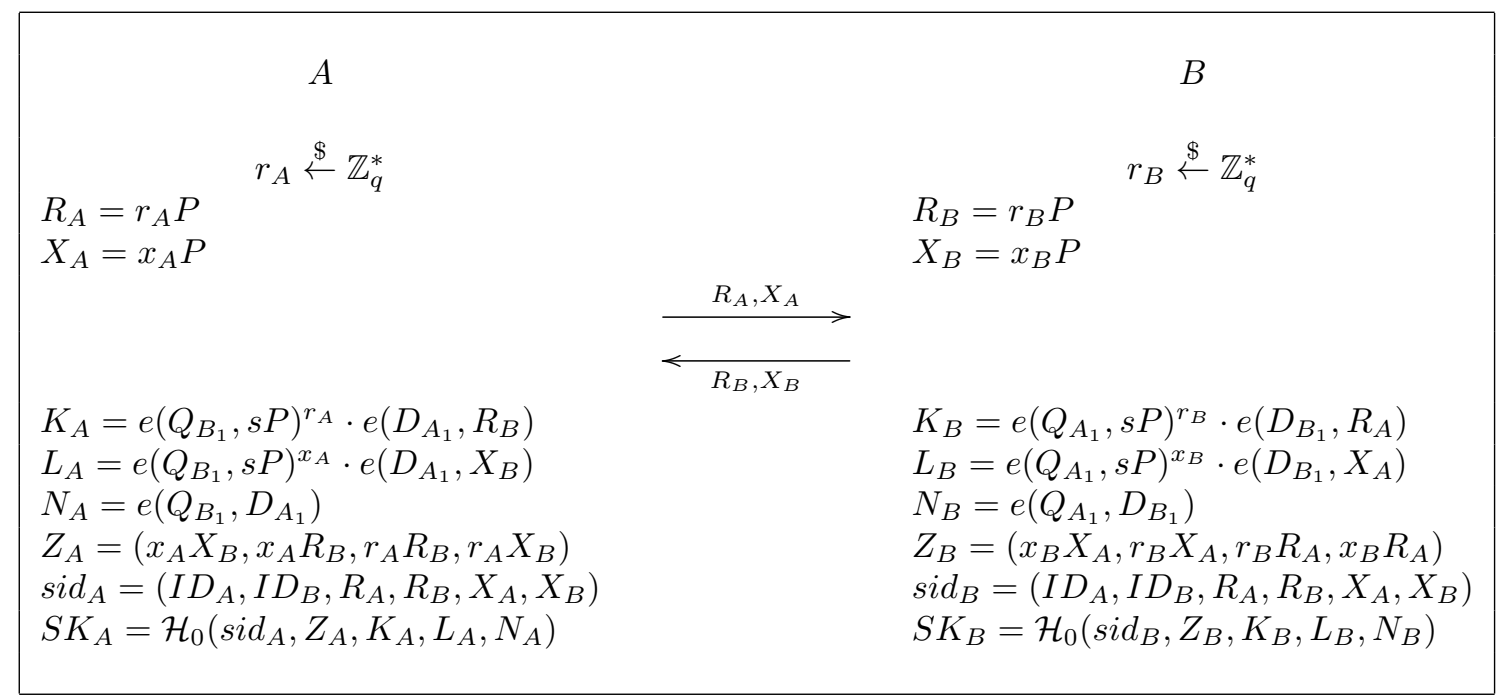

Figura 5.10: Protocolo $L B G$, seguro sob o problema Gap-BDH.

\section{Corretude}

$K_{A_{1}}=e\left(Q_{B_{1}}, s P\right)^{r_{A}} \cdot e\left(D_{A_{1}}, R_{B}\right)=e\left(Q_{B_{1}}, s P\right)^{r_{A}} \cdot e\left(s Q_{A_{1}}, r_{B} P\right)=e\left(Q_{B_{1}}, r_{A} P\right)^{s} \cdot e\left(Q_{A_{1}}, r_{B} P\right)^{s}=$ $e\left(s Q_{B_{1}}, r_{A} P\right) \cdot e\left(Q_{A_{1}}, s P\right)^{r_{B}}=e\left(D_{B_{1}}, R_{A}\right) \cdot e\left(Q_{A_{1}}, s P\right)^{r_{B}}=K_{B_{1}}$

$K_{A_{2}}=e\left(Q_{B_{2}}, s P\right)^{r_{A}} \cdot e\left(D_{A_{2}}, R_{B}\right)=e\left(Q_{B_{2}}, s P\right)^{r_{A}} \cdot e\left(s Q_{A_{2}}, r_{B} P\right)=e\left(Q_{B_{2}}, r_{A} P\right)^{s} \cdot e\left(Q_{A_{2}}, r_{B} P\right)^{s}=$ $e\left(s Q_{B_{2}}, r_{A} P\right) \cdot e\left(Q_{A_{2}}, s P\right)^{r_{B}}=e\left(D_{B_{2}}, R_{A}\right) \cdot e\left(Q_{A_{2}}, s P\right)^{r_{B}}=K_{B_{2}}$

$L_{A_{1}}=e\left(Q_{B_{1}}, s P\right)^{x_{A}} \cdot e\left(D_{A_{1}}, X_{B}\right)=e\left(Q_{B_{1}}, s P\right)^{x_{A}} \cdot e\left(s Q_{A_{1}}, x_{B} P\right)=e\left(Q_{B_{1}}, x_{A} P\right)^{s} \cdot e\left(Q_{A_{1}}, x_{B} P\right)^{s}=$ $e\left(s Q_{B_{1}}, x_{A} P\right) \cdot e\left(Q_{A_{1}}, s P\right)^{x_{B}}=e\left(D_{B_{1}}, X_{A}\right) \cdot e\left(Q_{A_{1}}, s P\right)^{x_{B}}=L_{B_{1}}$

$L_{A_{2}}=e\left(Q_{B_{2}}, s P\right)^{x_{A}} \cdot e\left(D_{A_{2}}, X_{B}\right)=e\left(Q_{B_{2}}, s P\right)^{x_{A}} \cdot e\left(s Q_{A_{2}}, x_{B} P\right)=e\left(Q_{B_{2}}, x_{A} P\right)^{s} \cdot e\left(Q_{A_{2}}, x_{B} P\right)^{s}=$ $e\left(s Q_{B_{2}}, x_{A} P\right) \cdot e\left(Q_{A_{2}}, s P\right)^{x_{B}}=e\left(D_{B_{2}}, X_{A}\right) \cdot e\left(Q_{A_{2}}, s P\right)^{x_{B}}=L_{B_{2}}$

$$
\begin{gathered}
N_{A_{1}}=e\left(Q_{B_{1}}, D_{A_{1}}\right)=e\left(Q_{B_{1}}, s Q_{A_{1}}\right)=e\left(Q_{B_{1}}, Q_{A_{1}}\right)^{s}=e\left(s Q_{B_{1}}, Q_{A_{1}}\right)=e\left(D_{B_{1}}, Q_{A_{1}}\right)=N_{B_{1}} \\
N_{A_{2}}=e\left(Q_{B_{2}}, D_{A_{2}}\right)=e\left(Q_{B_{2}}, s Q_{A_{2}}\right)=e\left(Q_{B_{2}}, Q_{A_{2}}\right)^{s}=e\left(s Q_{B_{2}}, Q_{A_{2}}\right)=e\left(D_{B_{2}}, Q_{A_{2}}\right)=N_{B_{2}} \\
Z_{A}=\left(x_{A} X_{B}, x_{A} R_{B}, r_{A} R_{B}, r_{A} X_{B}\right)=\left(x_{A} x_{B} P, x_{A} r_{B} P, r_{A} r_{B} P, r_{A} x_{B} P\right)=\left(x_{B} X_{A}, r_{B} X_{A}, r_{B} R_{A}, x_{B} R_{A}\right)= \\
Z_{B}
\end{gathered}
$$

\subsubsection{Protocolo Goya-Okida-Terada}

Em Goya et al. (2010) foram propostos dois protocolos, representados nas Figuras 5.11 e 5.12, que são melhorias dos protocolos apresentados em Lippold et al. (2009). Ambos são demonstrados seguros no modelo de segurança LBG e sob as hipóteses dos problemas BDH e Gap-BDH, respectivamente. 


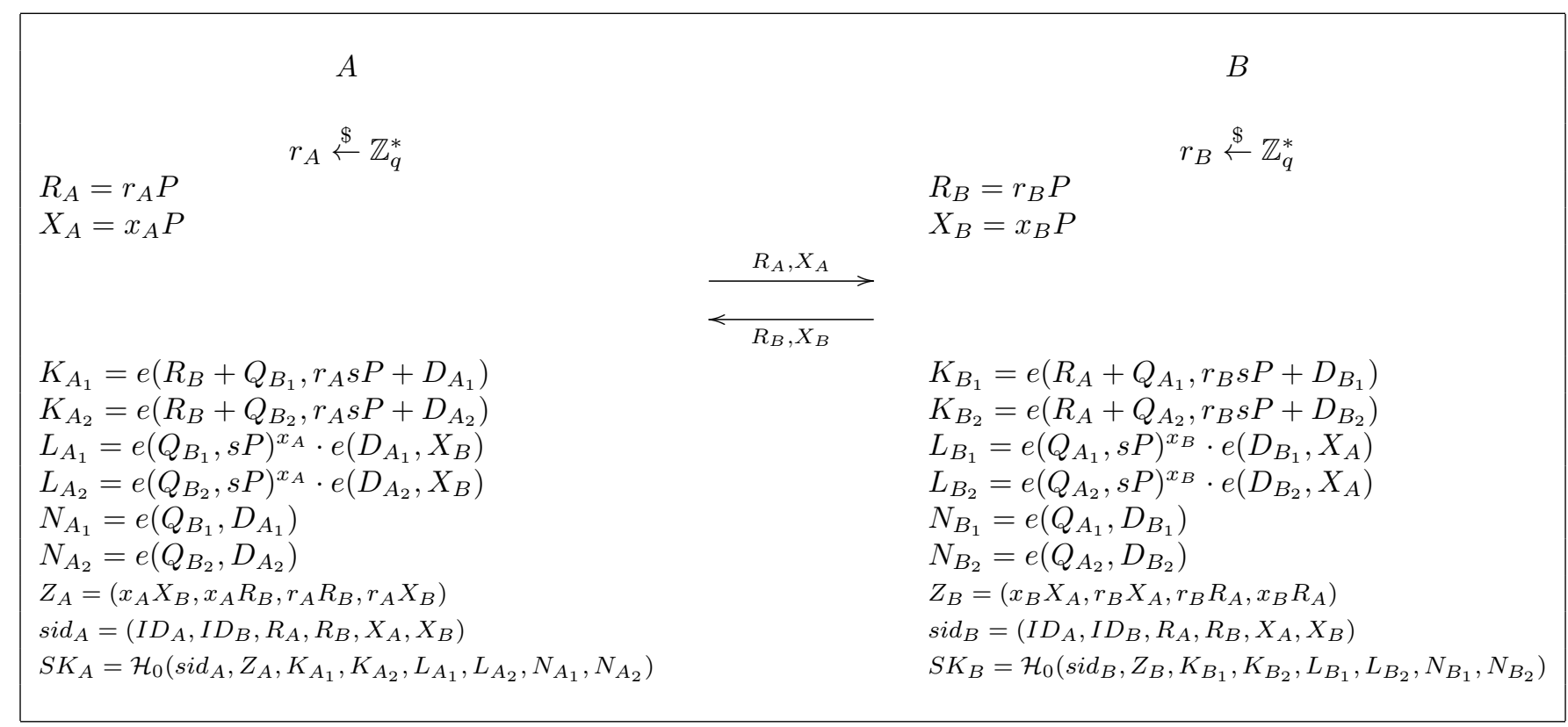

Figura 5.11: Protocolo GOT, seguro sob o problema $B D H$.

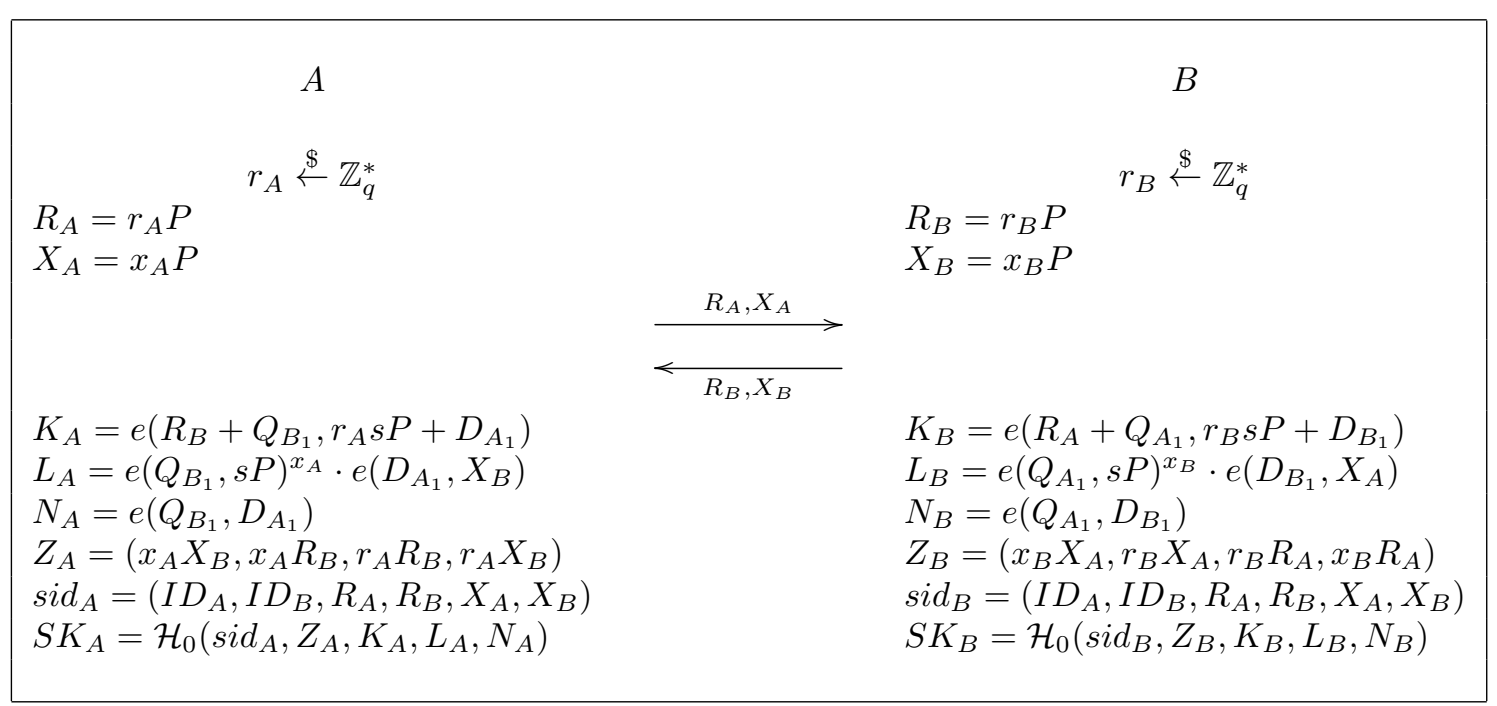

Figura 5.12: Protocolo GOT, seguro sob o problema Gap-BDH.

\section{Corretude}

$K_{A_{1}}=e\left(R_{B}+Q_{B_{1}}, r_{A} s P+D_{A_{1}}\right)=e\left(r_{B} P+Q_{B_{1}}, r_{A} s P+s Q_{A_{1}}\right)=e\left(r_{B} P+Q_{B_{1}}, r_{A} P+Q_{A_{1}}\right)^{s}=$ $e\left(r_{B} s P+s Q_{B_{1}}, r_{A} P+Q_{A_{1}}\right)=e\left(r_{B} s P+D_{B_{1}}, R_{A}+Q_{A_{1}}\right)=K_{B_{1}}$

$K_{A_{2}}=e\left(R_{B}+Q_{B_{2}}, r_{A} s P+D_{A_{2}}\right)=e\left(r_{B} P+Q_{B_{2}}, r_{A} s P+s Q_{A_{2}}\right)=e\left(r_{B} P+Q_{B_{2}}, r_{A} P+Q_{A_{2}}\right)^{s}=$ $e\left(r_{B} s P+s Q_{B_{2}}, r_{A} P+Q_{A_{2}}\right)=e\left(r_{B} s P+D_{B_{2}}, R_{A}+Q_{A_{2}}\right)=K_{B_{2}}$

$L_{A_{1}}=e\left(Q_{B_{1}}, s P\right)^{x_{A}} \cdot e\left(D_{A_{1}}, X_{B}\right)=e\left(Q_{B_{1}}, s P\right)^{x_{A}} \cdot e\left(s Q_{A_{1}}, x_{B} P\right)=e\left(Q_{B_{1}}, x_{A} P\right)^{s} \cdot e\left(Q_{A_{1}}, x_{B} P\right)^{s}=$ $e\left(s Q_{B_{1}}, x_{A} P\right) \cdot e\left(Q_{A_{1}}, s P\right)^{x_{B}}=e\left(D_{B_{1}}, X_{A}\right) \cdot e\left(Q_{A_{1}}, s P\right)^{x_{B}}=L_{B_{1}}$

$L_{A_{2}}=e\left(Q_{B_{2}}, s P\right)^{x_{A}} \cdot e\left(D_{A_{2}}, X_{B}\right)=e\left(Q_{B_{2}}, s P\right)^{x_{A}} \cdot e\left(s Q_{A_{2}}, x_{B} P\right)=e\left(Q_{B_{2}}, x_{A} P\right)^{s} \cdot e\left(Q_{A_{2}}, x_{B} P\right)^{s}=$ $e\left(s Q_{B_{2}}, x_{A} P\right) \cdot e\left(Q_{A_{2}}, s P\right)^{x_{B}}=e\left(D_{B_{2}}, X_{A}\right) \cdot e\left(Q_{A_{2}}, s P\right)^{x_{B}}=L_{B_{2}}$ 


$$
\begin{gathered}
N_{A_{1}}=e\left(Q_{B_{1}}, D_{A_{1}}\right)=e\left(Q_{B_{1}}, s Q_{A_{1}}\right)=e\left(Q_{B_{1}}, Q_{A_{1}}\right)^{s}=e\left(s Q_{B_{1}}, Q_{A_{1}}\right)=e\left(D_{B_{1}}, Q_{A_{1}}\right)=N_{B_{1}} \\
N_{A_{2}}=e\left(Q_{B_{2}}, D_{A_{2}}\right)=e\left(Q_{B_{2}}, s Q_{A_{2}}\right)=e\left(Q_{B_{2}}, Q_{A_{2}}\right)^{s}=e\left(s Q_{B_{2}}, Q_{A_{2}}\right)=e\left(D_{B_{2}}, Q_{A_{2}}\right)=N_{B_{2}} \\
Z_{A}=\left(x_{A} X_{B}, x_{A} R_{B}, r_{A} R_{B}, r_{A} X_{B}\right)=\left(x_{A} x_{B} P, x_{A} r_{B} P, r_{A} r_{B} P, r_{A} x_{B} P\right)=\left(x_{B} X_{A}, r_{B} X_{A}, r_{B} R_{A}, x_{B} R_{A}\right)= \\
Z_{B}
\end{gathered}
$$

\subsubsection{Protocolo Goya-Nakamura-Terada-3}

Goya (2011) propôs o protocolo representado na Figura 5.13, que é uma versão mais eficiente do protocolo GOT-LBG (Goya et al., 2010). Foi demonstrado seguro no modelo de segurança LBG, sob a hipótese do problema BDH.

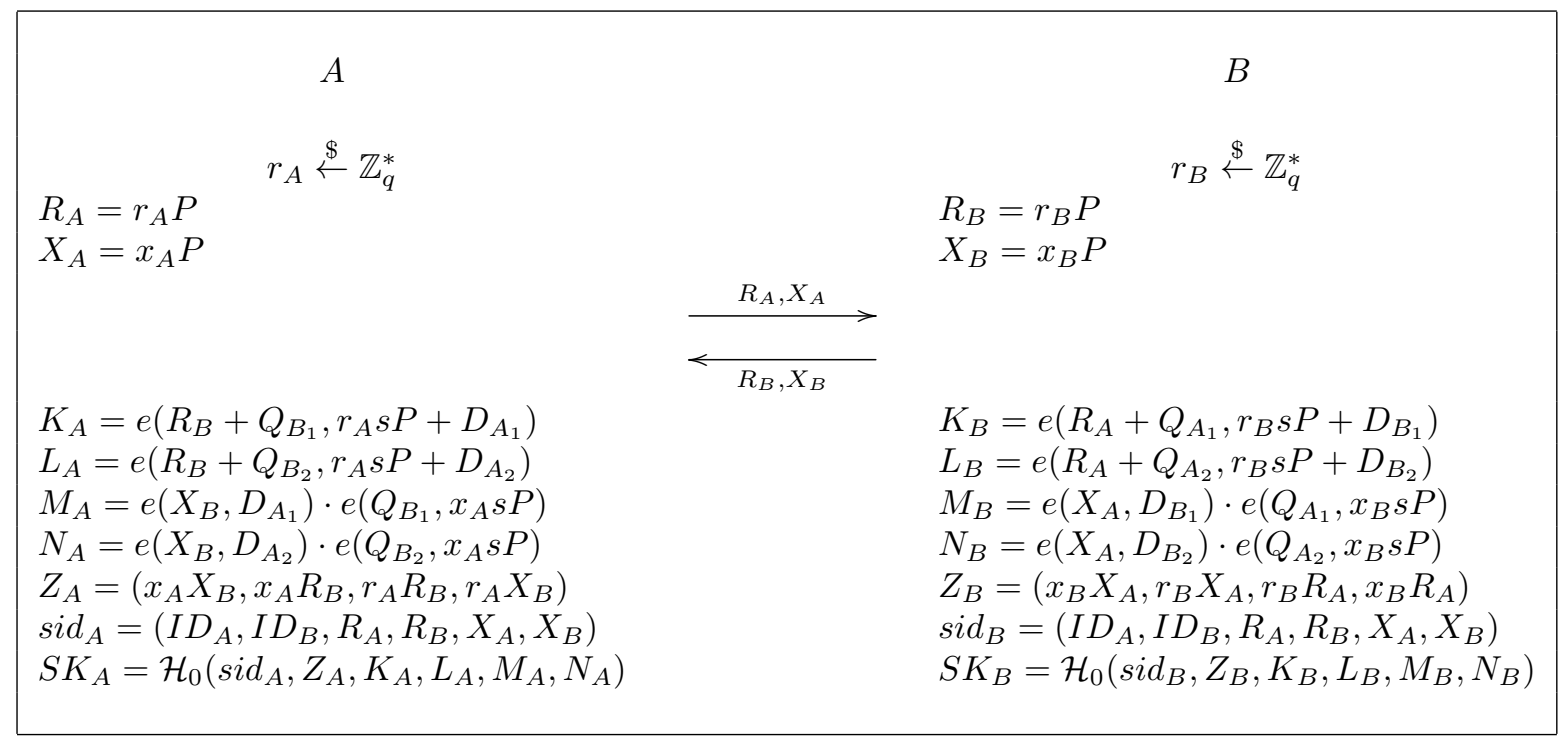

Figura 5.13: Protocolo GNT3, seguro sob o problema $B D H$.

\section{Corretude}

$K_{A}=e\left(R_{B}+Q_{B_{1}}, r_{A} s P+D_{A_{1}}\right)=e\left(r_{B} P+Q_{B_{1}}, r_{A} s P+s Q_{A_{1}}\right)=e\left(r_{B} P+Q_{B_{1}}, r_{A} P+Q_{A_{1}}\right)^{s}=$ $e\left(r_{B} s P+s Q_{B_{1}}, r_{A} P+Q_{A_{1}}\right)=e\left(r_{B} s P+D_{B_{1}}, R_{A}+Q_{A_{1}}\right)=K_{B}$

$L_{A}=e\left(R_{B}+Q_{B_{2}}, r_{A} s P+D_{A_{2}}\right)=e\left(r_{B} P+Q_{B_{2}}, r_{A} s P+s Q_{A_{2}}\right)=e\left(r_{B} P+Q_{B_{2}}, r_{A} P+Q_{A_{2}}\right)^{s}=$ $e\left(r_{B} s P+s Q_{B_{2}}, r_{A} P+Q_{A_{2}}\right)=e\left(r_{B} s P+D_{B_{2}}, R_{A}+Q_{A_{2}}\right)=L_{B}$

$M_{A}=e\left(X_{B}, D_{A_{1}}\right) \cdot e\left(Q_{B_{1}}, x_{A} s P\right)=e\left(x_{B} P, s Q_{A_{1}}\right) \cdot e\left(Q_{B_{1}}, x_{A} s P\right)=e\left(x_{B} P, Q_{A_{1}}\right)^{s} \cdot e\left(Q_{B_{1}}, x_{A} P\right)^{s}=$ $e\left(x_{B} s P, Q_{A_{1}}\right) \cdot e\left(s Q_{B_{1}}, x_{A} P\right)=e\left(x_{B} s P, Q_{A_{1}}\right) \cdot e\left(D_{B_{1}}, X_{A}\right)=M_{B}$

$M_{A}=e\left(X_{B}, D_{A_{2}}\right) \cdot e\left(Q_{B_{2}}, x_{A} s P\right)=e\left(x_{B} P, s Q_{A_{2}}\right) \cdot e\left(Q_{B_{2}}, x_{A} s P\right)=e\left(x_{B} P, Q_{A_{2}}\right)^{s} \cdot e\left(Q_{B_{2}}, x_{A} P\right)^{s}=$ $e\left(x_{B} s P, Q_{A_{2}}\right) \cdot e\left(s Q_{B_{2}}, x_{A} P\right)=e\left(x_{B} s P, Q_{A_{2}}\right) \cdot e\left(D_{B_{2}}, X_{A}\right)=M_{B}$ $Z_{B}$

$Z_{A}=\left(x_{A} X_{B}, x_{A} R_{B}, r_{A} R_{B}, r_{A} X_{B}\right)=\left(x_{A} x_{B} P, x_{A} r_{B} P, r_{A} r_{B} P, r_{A} x_{B} P\right)=\left(x_{B} X_{A}, r_{B} X_{A}, r_{B} R_{A}, x_{B} R_{A}\right)=$

\subsubsection{Protocolo Goya-Nakamura-Terada-4}

Outro protocolo proposto em Goya (2011), representado na Figura 5.14, foi demonstrado seguro no modelo de segurança $\mathrm{SJ}^{+}$, sob a hipótese do problema BDH. 


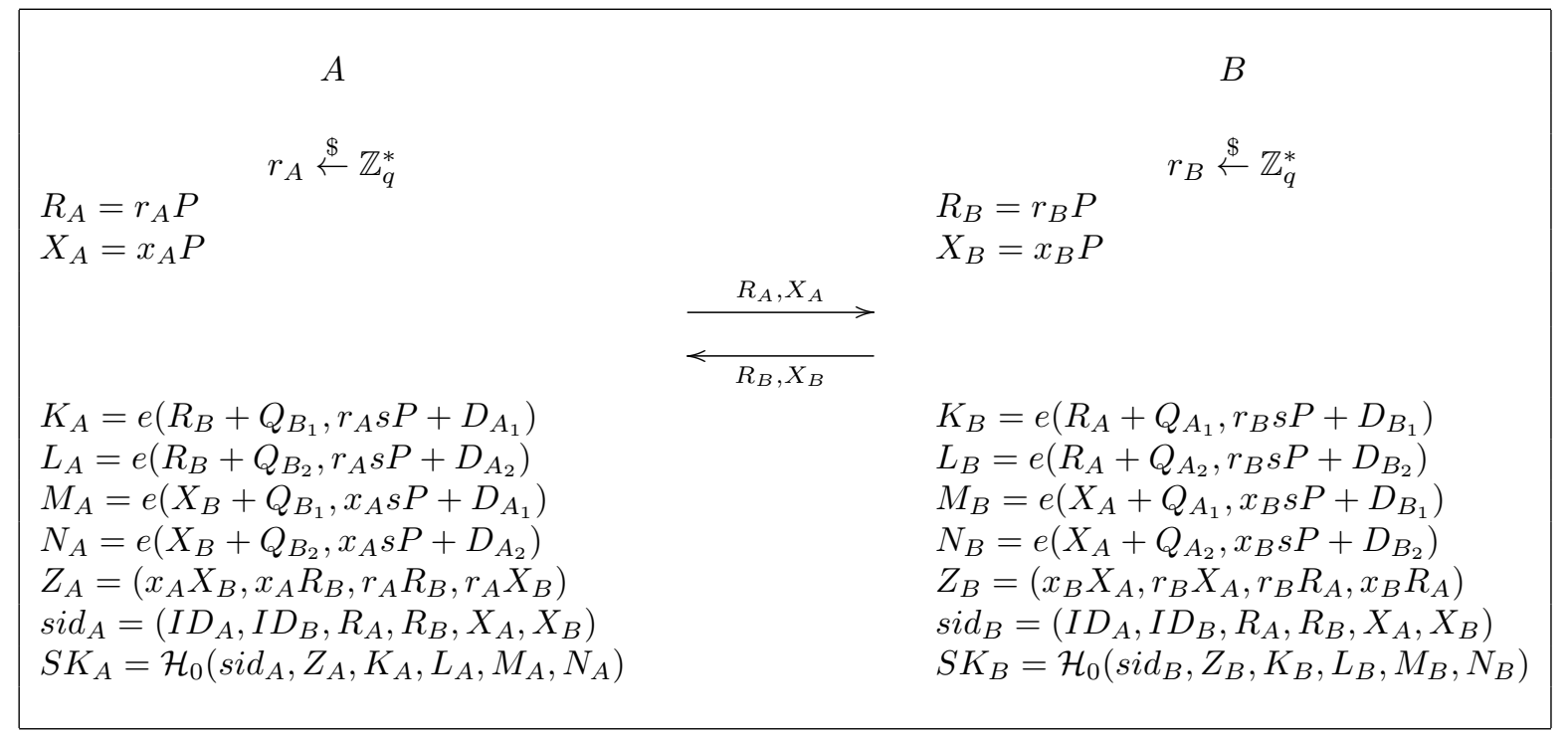

Figura 5.14: Protocolo GNT4, seguro sob o problema BDH.

\section{Corretude}

$K_{A}=e\left(R_{B}+Q_{B_{1}}, r_{A} s P+D_{A_{1}}\right)=e\left(r_{B} P+Q_{B_{1}}, r_{A} s P+s Q_{A_{1}}\right)=e\left(r_{B} P+Q_{B_{1}}, r_{A} P+Q_{A_{1}}\right)^{s}=$ $e\left(r_{B} s P+s Q_{B_{1}}, r_{A} P+Q_{A_{1}}\right)=e\left(r_{B} s P+D_{B_{1}}, R_{A}+Q_{A_{1}}\right)=K_{B}$

$L_{A}=e\left(R_{B}+Q_{B_{2}}, r_{A} s P+D_{A_{2}}\right)=e\left(r_{B} P+Q_{B_{2}}, r_{A} s P+s Q_{A_{2}}\right)=e\left(r_{B} P+Q_{B_{2}}, r_{A} P+Q_{A_{2}}\right)^{s}=$ $e\left(r_{B} s P+s Q_{B_{2}}, r_{A} P+Q_{A_{2}}\right)=e\left(r_{B} s P+D_{B_{2}}, R_{A}+Q_{A_{2}}\right)=L_{B}$

$M_{A}=e\left(X_{B}+Q_{B_{1}}, x_{A} s P+D_{A_{1}}\right)=e\left(x_{B} P+Q_{B_{1}}, x_{A} s P+s Q_{A_{1}}\right)=e\left(x_{B} P+Q_{B_{1}}, x_{A} P+Q_{A_{1}}\right)^{s}=$ $e\left(x_{B} s P+s Q_{B_{1}}, x_{A} P+Q_{A_{1}}\right)=e\left(x_{B} s P+D_{B_{1}}, X_{A}+Q_{A_{1}}\right)=M_{B}$

$N_{A}=e\left(X_{B}+Q_{B_{2}}, x_{A} s P+D_{A_{2}}\right)=e\left(x_{B} P+Q_{B_{2}}, x_{A} s P+s Q_{A_{2}}\right)=e\left(x_{B} P+Q_{B_{2}}, x_{A} P+Q_{A_{2}}\right)^{s}=$ $e\left(x_{B} s P+s Q_{B_{2}}, x_{A} P+Q_{A_{2}}\right)=e\left(x_{B} s P+D_{B_{2}}, X_{A}+Q_{A_{2}}\right)=N_{B}$ $Z_{B}$

$Z_{A}=\left(x_{A} X_{B}, x_{A} R_{B}, r_{A} R_{B}, r_{A} X_{B}\right)=\left(x_{A} x_{B} P, x_{A} r_{B} P, r_{A} r_{B} P, r_{A} x_{B} P\right)=\left(x_{B} X_{A}, r_{B} X_{A}, r_{B} R_{A}, x_{B} R_{A}\right)=$

\subsubsection{Protocolo Goya-Nakamura-Terada-1}

O protocolo de Goya et al. (2011), representado na Figura 5.15, foi demonstrado seguro no modelo de segurança Mal-LBG, sob a hipótese do problema Gap-BDH. 


\begin{tabular}{|c|c|c|}
\hline$A$ & & B \\
\hline $\begin{array}{l}r_{A} \stackrel{\$}{\leftarrow} \mathbb{Z}_{q}^{*} \\
R_{A}=r_{A} P \\
X_{A}=x_{A} P\end{array}$ & & $\begin{array}{l}r_{B} \stackrel{\$}{\leftarrow} \mathbb{Z}_{q}^{*} \\
R_{B}=r_{B} P \\
X_{B}=x_{B} P\end{array}$ \\
\hline & $R_{A}, X_{A}$ & \\
\hline $\begin{array}{l}K_{A}=e\left(R_{B}+Q_{B_{1}}, r_{A} s P+D_{A_{1}}\right) \\
L_{A}=e\left(R_{B}+Q_{B_{2}}, r_{A} s P+D_{A_{2}}\right) \\
M_{A}=e\left(X_{B}, D_{A_{1}}\right) \cdot e\left(Q_{B_{1}}, x_{A} s P\right) \\
Z_{A}=\left(x_{A} X_{B}, x_{A} R_{B}, r_{A} R_{B}, r_{A} X_{B}\right) \\
\operatorname{sid}_{A}=\left(I D_{A}, I D_{B}, R_{A}, R_{B}, X_{A}, X_{B}\right) \\
S K_{A}=\mathcal{H}_{0}\left(\operatorname{sid}_{A}, Z_{A}, K_{A}, L_{A}, M_{A}\right)\end{array}$ & $R_{B}, X_{B}$ & $\begin{array}{l}K_{B}=e\left(R_{A}+Q_{A_{1}}, r_{B} s P+D_{B_{1}}\right) \\
L_{B}=e\left(R_{A}+Q_{A_{2}}, r_{B} s P+D_{B_{2}}\right) \\
M_{B}=e\left(X_{A}, D_{B_{1}}\right) \cdot e\left(Q_{A_{1}}, x_{B} s P\right) \\
Z_{B}=\left(x_{B} X_{A}, r_{B} X_{A}, r_{B} R_{A}, x_{B} R_{A}\right) \\
s_{B}=\left(I d_{B}, I D_{B}, R_{A}, R_{B}, X_{A}, X_{B}\right) \\
S K_{B}=\mathcal{H}_{0}\left(\operatorname{sid}_{B}, Z_{B}, K_{B}, L_{B}, M_{B}\right)\end{array}$ \\
\hline
\end{tabular}

Figura 5.15: Protocolo GNT1, seguro sob o problema Gap-BDH.

\section{Corretude}

$$
\begin{gathered}
K_{A}=e\left(R_{B}+Q_{B_{1}}, r_{A} s P+D_{A_{1}}\right)=e\left(r_{B} P+Q_{B_{1}}, r_{A} s P+s Q_{A_{1}}\right)=e\left(r_{B} P+Q_{B_{1}}, r_{A} P+Q_{A_{1}}\right)^{s}= \\
e\left(r_{B} s P+s Q_{B_{1}}, r_{A} P+Q_{A_{1}}\right)=e\left(r_{B} s P+D_{B_{1}}, R_{A}+Q_{A_{1}}\right)=K_{B} \\
L_{A}=e\left(R_{B}+Q_{B_{2}}, r_{A} s P+D_{A_{2}}\right)=e\left(r_{B} P+Q_{B_{2}}, r_{A} s P+s Q_{A_{2}}\right)=e\left(r_{B} P+Q_{B_{2}}, r_{A} P+Q_{A_{2}}\right)^{s}= \\
e\left(r_{B} s P+s Q_{B_{2}}, r_{A} P+Q_{A_{2}}\right)=e\left(r_{B} s P+D_{B_{2}}, R_{A}+Q_{A_{2}}\right)=L_{B} \\
\quad M_{A}=e\left(X_{B}, D_{A_{1}}\right) \cdot e\left(Q_{B_{1}}, x_{A} s P\right)=e\left(x_{B} P, s Q_{A_{1}}\right) \cdot e\left(Q_{B_{1}}, x_{A} s P\right)=e\left(x_{B} P, Q_{A_{1}}\right)^{s} \cdot e\left(Q_{B_{1}}, x_{A} P\right)^{s}= \\
e\left(x_{B} s P, Q_{A_{1}}\right) \cdot e\left(s Q_{B_{1}}, x_{A} P\right)=e\left(x_{B} s P, Q_{A_{1}}\right) \cdot e\left(D_{B_{1}}, X_{A}\right)=M_{B} \\
\quad Z_{A}=\left(x_{A} X_{B}, x_{A} R_{B}, r_{A} R_{B}, r_{A} X_{B}\right)=\left(x_{A} x_{B} P, x_{A} r_{B} P, r_{A} r_{B} P, r_{A} x_{B} P\right)=\left(x_{B} X_{A}, r_{B} X_{A}, r_{B} R_{A}, x_{B} R_{A}\right)= \\
Z_{B}
\end{gathered}
$$

\subsubsection{Protocolo Goya-Nakamura-Terada-2}

Também proposto em Goya (2011), o protocolo representado na Figura 5.16 foi demonstrado seguro no modelo de segurança Mal-SJ ${ }^{+}$, sob a hipótese do problema Gap-BDH.

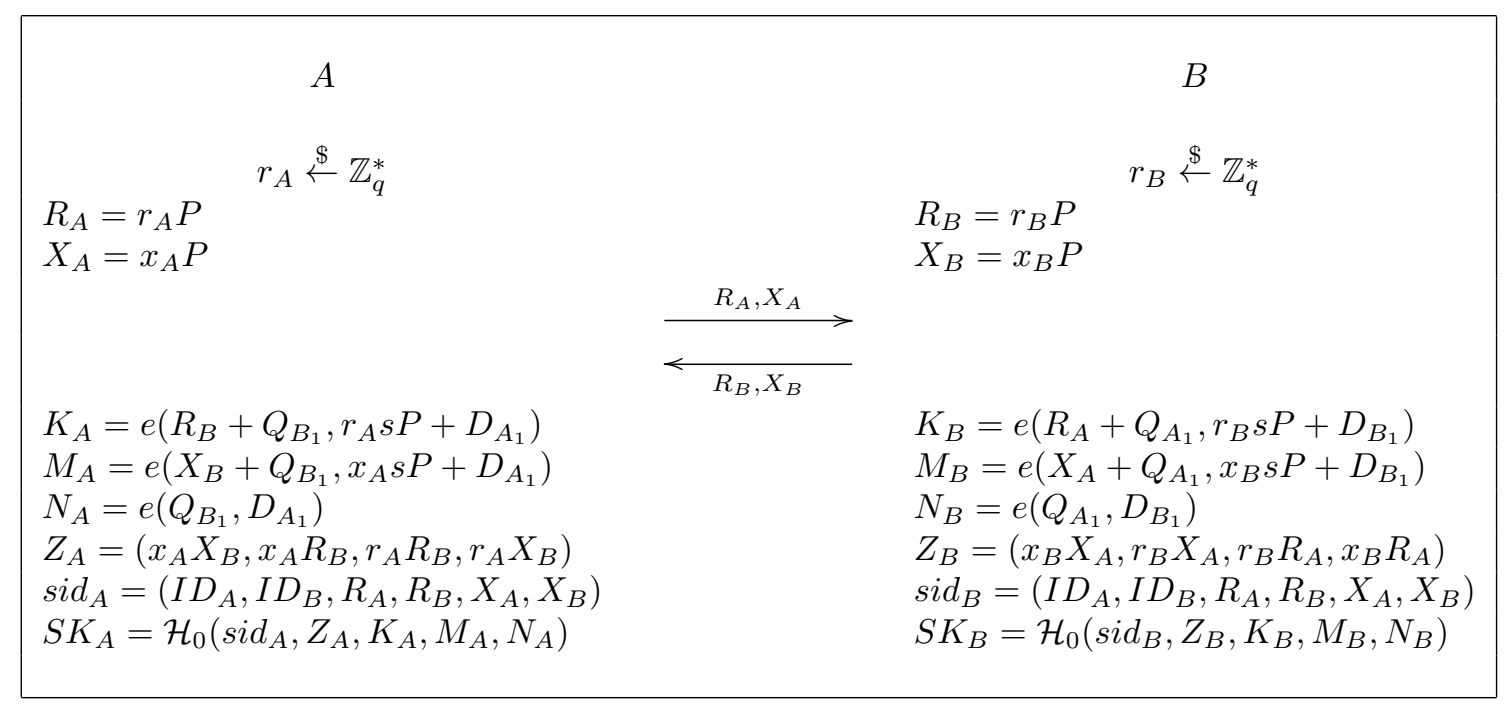

Figura 5.16: Protocolo GNT2, seguro sob o problema Gap-BDH. 


\section{Corretude}

$$
\begin{aligned}
& K_{A}=e\left(R_{B}+Q_{B_{1}}, r_{A} s P+D_{A_{1}}\right)=e\left(r_{B} P+Q_{B_{1}}, r_{A} s P+s Q_{A_{1}}\right)=e\left(r_{B} P+Q_{B_{1}}, r_{A} P+Q_{A_{1}}\right)^{s}= \\
& e\left(r_{B} s P+s Q_{B_{1}}, r_{A} P+Q_{A_{1}}\right)=e\left(r_{B} s+D_{B_{1}}, R_{A}+Q_{A_{1}}\right)=K_{B} \\
& M_{A}=e\left(X_{B}+Q_{B_{1}}, x_{A} s P+D_{A_{1}}\right)=e\left(x_{B} P+Q_{B_{1}}, x_{A} s P+s Q_{A_{1}}\right)=e\left(x_{B} P+Q_{B_{1}}, x_{A} P+Q_{A_{1}}\right)^{s}= \\
& e\left(x_{B} s P+s Q_{B_{1}}, x_{A} P+Q_{A_{1}}\right)=e\left(x_{B} s P+D_{B_{1}}, X_{A}+Q_{A_{1}}\right)=M_{B} \\
& N_{A}=e\left(Q_{B_{1}}, D_{A_{1}}\right)=e\left(Q_{B_{1}}, s Q_{A_{1}}\right)=e\left(Q_{B_{1}}, Q_{A_{1}}\right)^{s}=e\left(s Q_{B_{1}}, Q_{A_{1}}\right)=e\left(D_{B_{1}}, Q_{A_{1}}\right)=N_{B} \\
& Z_{A}=\left(x_{A} X_{B}, x_{A} R_{B}, r_{A} R_{B}, r_{A} X_{B}\right)=\left(x_{A} x_{B} P, x_{A} r_{B} P, r_{A} r_{B} P, r_{A} x_{B} P\right)=\left(x_{B} X_{A}, r_{B} X_{A}, r_{B} R_{A}, x_{B} R_{A}\right)= \\
& Z_{B}
\end{aligned}
$$

\subsection{Adaptação para emparelhamento assimétrico}

Conforme os experimentos que serão discutidos na Seção 6.3, operações com emparelhamento assimétrico são mais eficientes computacionalmente do que operações com emparelhamento simétrico. Contudo, a implementação de protocolos utilizando emparelhamento assimétrico requer algumas adaptações.

$\mathrm{Na}$ etapa de inicialização, a autoridade do sistema deve selecionar três grupos cíclicos $\mathbb{G}_{1}$ e $\mathbb{G}_{2}$ aditivos, e $\mathbb{G}_{T}$ multiplicativo (todos de ordem $q$ ), e dois geradores, $P \in \mathbb{G}_{1}$ e $P^{\prime} \in \mathbb{G}_{2}$. Considera-se $e: \mathbb{G}_{1} \times \mathbb{G}_{2} \rightarrow \mathbb{G}_{T}$, um emparelhamento assimétrico admissível, e um parâmetro de segurança $k$.

A autoridade também escolhe sua chave secreta $s \stackrel{\$}{\leftarrow} \mathbb{Z}_{q}^{*}$, e calcula sua chave pública como sendo o par $\left\langle s P, s P^{\prime}\right\rangle$. Por fim, seleciona também as funções de hash $\mathcal{H}_{0}, \mathcal{H}_{1}, \mathcal{H}_{1}^{\prime}$, tais que $\mathcal{H}_{0}$ : $\{0,1\}^{*} \rightarrow\{0,1\}^{k}, \mathcal{H}_{1}:\{0,1\}^{*} \rightarrow \mathbb{G}_{1}$ e $\mathcal{H}_{1}^{\prime}:\{0,1\}^{*} \rightarrow \mathbb{G}_{2}$, e torna públicos os parâmetros params' $=\left\langle k, \mathbb{G}_{1}, \mathbb{G}_{2}, \mathbb{G}_{T}, q, e, P, P^{\prime} s P, s P^{\prime}, \mathcal{H}_{0}, \mathcal{H}_{1}, \mathcal{H}_{1}^{\prime}\right\rangle$.

No caso dos protocolos com parâmetros estendidos, a autoridade do sistema também seleciona as funções de $h$ ash adicionais $\mathcal{H}_{2}, \mathcal{H}_{2}^{\prime}$, tais que $\mathcal{H}_{2}:\{0,1\}^{*} \rightarrow \mathbb{G}_{1}$ e $\mathcal{H}_{2}^{\prime}:\{0,1\}^{*} \rightarrow \mathbb{G}_{2}$. Logo após, divulga os parâmetros públicos params_ext' $=\left\langle k, \mathbb{G} 1, \mathbb{G}_{2}, \mathbb{G}_{T}, q, e, P, P^{\prime}, s P, s P^{\prime}, \mathcal{H}_{0}, \mathcal{H}_{1}, \mathcal{H}_{2}, \mathcal{H}_{1}^{\prime}, \mathcal{H}_{2}^{\prime}\right\rangle$.

A chave pública de um usuário $U$ passa ser o par $\left\langle Q_{U_{1}}, Q_{U_{1}}^{\prime}\right\rangle$, tal que $Q_{U_{1}}=\mathcal{H}_{1}\left(I D_{U}\right)$ e $Q_{U_{1}}^{\prime}=\mathcal{H}_{1}^{\prime}\left(I D_{U}\right)$. Assim, a autoridade do sistema deve calcular a chave secreta ${ }^{3}$ de $U$ como sendo o $\operatorname{par}\left\langle S_{U_{1}}, S_{U_{1}}^{\prime}\right\rangle$, onde $S_{U_{1}}=s Q_{U_{1}}$ e $S_{U_{1}}^{\prime}=s Q_{U_{1}}^{\prime}$. Os protocolos que utilizam parâmetros estendidos são adaptados de forma análoga, com adição dos valores públicos $\left\langle Q_{U_{2}}, Q_{U_{2}}^{\prime}\right\rangle$ e secretos $\left\langle S_{U_{2}}, S_{U_{2}}^{\prime}\right\rangle$, tais que $Q_{U_{2}}=\mathcal{H}_{2}\left(I D_{U}\right), Q_{U_{2}}^{\prime}=\mathcal{H}_{2}^{\prime}\left(I D_{U}\right)$ e $S_{U_{2}}=s Q_{U_{2}}, S_{U_{2}}^{\prime}=s Q_{U_{2}}^{\prime}$.

Finalmente, na fase de troca de mensagens, $U$ seleciona um valor secreto $r_{U} \stackrel{\$}{\leftarrow} \mathbb{Z}_{q}^{*}$ e calcula o $\operatorname{par}\left\langle R_{U}, R_{U}^{\prime}\right\rangle$, onde $R_{U}=r_{U} P$ e $R_{U}^{\prime}=r_{U} P^{\prime}$. Em protocolos no modelo sem certificado, $U$ também deve calcular $\left\langle X_{U}, X_{U}^{\prime}\right\rangle$, tais que $X_{U}=x_{U} P$ e $X_{U}^{\prime}=x_{U} P^{\prime}$.

\footnotetext{
${ }^{3}$ Chave secreta parcial, no caso dos protocolos no modelo de criptografia sem certificado.
} 


\section{Capítulo 6}

\section{Experimentos}

Após o levantamento dos protocolos de acordo de chave, exposto no Capítulo 5, apresentamos a metodologia e os resultados dos experimentos de medição de desempenho realizados.

\subsection{Cenário proposto}

O cenário proposto para os experimentos consiste em acordar uma chave secreta entre um dispositivo de baixo poder computacional (cliente) e um servidor, que funciona como a autoridade do sistema (PKG ou KGC). Neste ambiente não há distribuição prévia de segredos compartilhados. A comunicação de ambos acontece por meio de uma rede TCP/IP, utilizando os padrões IEEE 802.11g (para comunicação sem fio) e 100BASE-TX (Fast Ethernet), a depender do dispositivo.

Com o intuito de validar os resultados obtidos, foram escolhidos três dispositivos de poder baixo poder computacional para a realização dos experimentos: um smartphone, um tablet, e um singleboard computer voltado para sistemas embarcados, todos com processadores ARM. Tais dispositivos dispõem de sistema operacional Android ou Linux. A seguir são detalhadas as configurações dos equipamentos utilizados:

- Servidor (PC):

- Modelo: Compaq CQ-50 113BR

- Processador: Intel Core 2 Duo T5800, $2.0 \mathrm{GHz}$

- Memória RAM: 3 GB, DDR2, $800 \mathrm{MHz}$

- Conexão de rede: $100 \mathrm{Mbps}$, por cabo (100BASE-TX)

- Sistema operacional: Ubuntu 12.04 LTS, 32 bits

- Raspberry Pi (RPi):

- Processador: ARM1176JZF-S (instruções ARMv6, single core), $700 \mathrm{MHz}$

- Memória RAM: 256 MB, LPDDR2

- Conexão de rede: $100 \mathrm{Mbps,} \mathrm{por} \mathrm{cabo} \mathrm{(100BASE-TX)}$

- Sistema operacional: Debian "Wheezy" (armhf)

\section{- Smartphone (MM1):}

- Modelo: Motorola Milestone 1

- Processador: ARM Cortex-A8 (instruções ARMv6, single core), $600 \mathrm{MHz}$

- Memória RAM: 256 MB, LPDDR

- Conexão de rede: $54 \mathrm{Mbps}$, sem fio (IEEE 802.11g)

- Sistema operacional: Android 2.2 
- Tablet (GN7):

- Modelo: Google Nexus 7

- Processador: Cortex-A9 (instruções ARMv7, quad-core), $1.2 \mathrm{GHz}$

- Memória RAM: 1 GB, DDR3

- Conexão de rede: $54 \mathrm{Mbps}$, sem fio (IEEE 802.11g)

- Sistema operacional: Android 4.2

- Roteador Wi-Fi:

- Modelo: D-Link DIR-300

- Processador: Atheros AR2317, $183 \mathrm{MHz}$

- Memória RAM: 16 MB

- Conexão de rede: 4x 100 Mbps (cabo), 54 Mbps (sem fio)

- Sistema operacional: DD-WRT v24-sp2

As mensagens trocadas entre os dispositivos e o servidor passam por um roteador, que atua como servidor $\mathrm{DHCP}^{1}$ e fornece a rede sem fio. A Figura 6.1 representa este cenário.

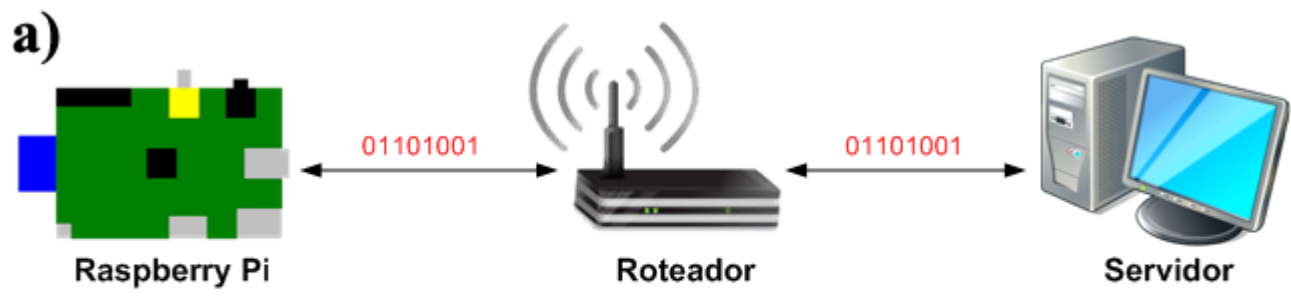

b)

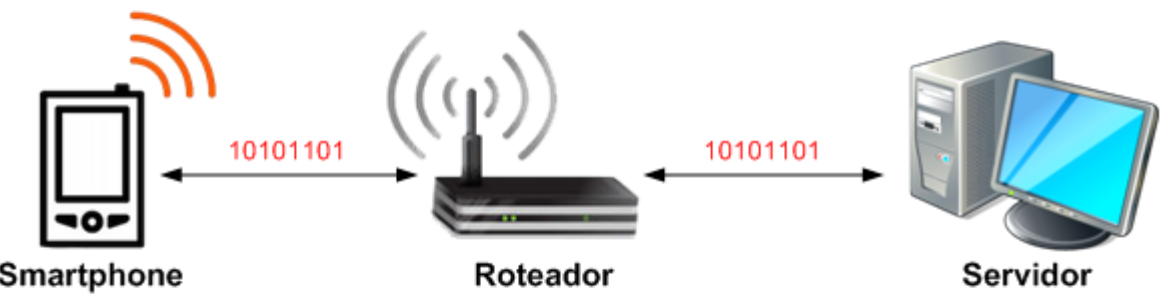

c)

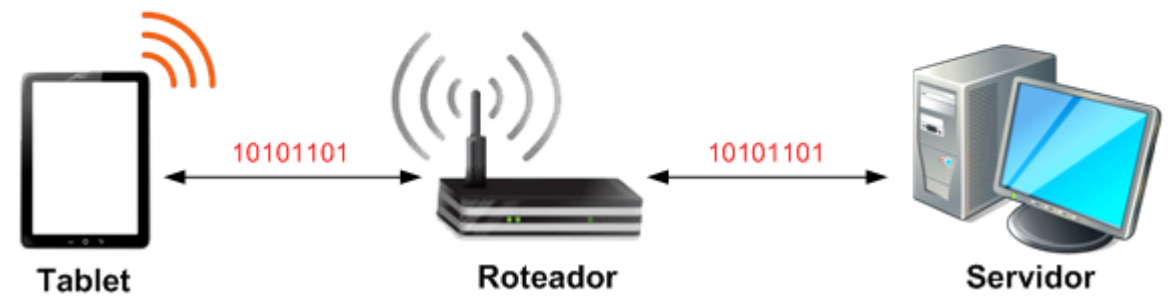

Figura 6.1: Cenário dos experimentos e comunicação entre os dispositivos: a) Raspberry Pi, b) Motorola Milestone 1, c) Google Nexus \%.

\subsection{Ambiente de desenvolvimento}

Para viabilizar a implementação dos protocolos, foi utilizada uma biblioteca criptográfica com suporte para operações sobre curvas elípticas e emparelhamentos bilineares. Escolhemos a RELICtoolkit (Aranha e Gouvêa, 2009), construída em linguagens C e Assembly, e com ênfase em eficiência. Tal biblioteca permite configurações flexíveis para níveis de segurança específicos e escolhas algorítmicas, além de suporte para diversas arquiteturas de processadores, como x86, ARM, AVR e MSP.

\footnotetext{
${ }^{1}$ Do inglês Dynamic Host Configuration Protocol, ou Protocolo de Configuração Dinâmica de Host.
} 
As implementações dos protocolos estudados foram escritas em linguagem $\mathrm{C}$, integrando-se de forma transparente à biblioteca RELIC-toolkit. A comunicação entre os dispositivos e o servidor é realizada através de sockets em C. Dentre as vantagens da utilização de sockets estão a fácil integração dos códigos construidos com as funções de comunicação em rede, comunicação TCP/IP direta, e facilidade em transmitir alguns tipos de dados complexos da RELIC-toolkit, já que não existem funções nativas que convertam tais tipos para strings e vice-versa.

O processo de compilação do código construído para os dispositivos com sistema operacional Android se deu com auxilio do Android Native Developement Kit (NDK). Trata-se de um framework para desenvolvimento de software, que permite escrever e compilar código nativo em linguagens $\mathrm{C}$ e $\mathrm{C}++$. Em geral, aplicativos para Android são construídos em linguagem de programação Java, e funcionam sobre a máquina virtual Dalvik (uma implementação da máquina virtual Java otimizada para Android). O código nativo produzido com auxílio do NDK pode ser executado a partir de aplicativos construídos em Java, através do Java Native Interface (JNI), como também pode ser executado de forma autônoma, utilizando-se um shell de comando.

Os experimentos deste trabalho foram executados a partir do shell de comando. Todavia, as chaves compartilhadas resultantes da execução dos protocolos de acordo de chave analisados podem ser facilmente convertidas para strings e enviadas para um aplicativo Android construido em Java, através de JNI. Desta forma, aplicativos do mundo real podem se beneficiar do código nativo gerado, que faz uso de uma das bibliotecas criptográficas mais eficientes disponíveis atualmente.

\subsection{Curvas elípticas e custo das operações}

A biblioteca RELIC-toolkit dispõe de um conjunto de curvas elípticas pré-definidas. Tais curvas se dividem em dois grupos: curvas elípticas sobre corpos binários (curvas binárias) e curvas elípticas sobre corpos primos (curvas primas). Nos interessam as curvas conhecidas como pairing-friendly, que permitem a construção de sistemas criptográficos que fazem uso de emparelhamentos bilineares (Freeman et al., 2010).

Escolhemos seis curvas elípticas que oferecem diferentes níveis de segurança, sendo três definidas sobre corpos binários e três definidas sobre corpos primos. A Figura 6.2 apresenta as curvas selecionadas e seus respectivos níveis de segurança. Conservamos a nomenclatura atribuída às curvas elípticas, de acordo com a documentação da RELIC-toolkit.

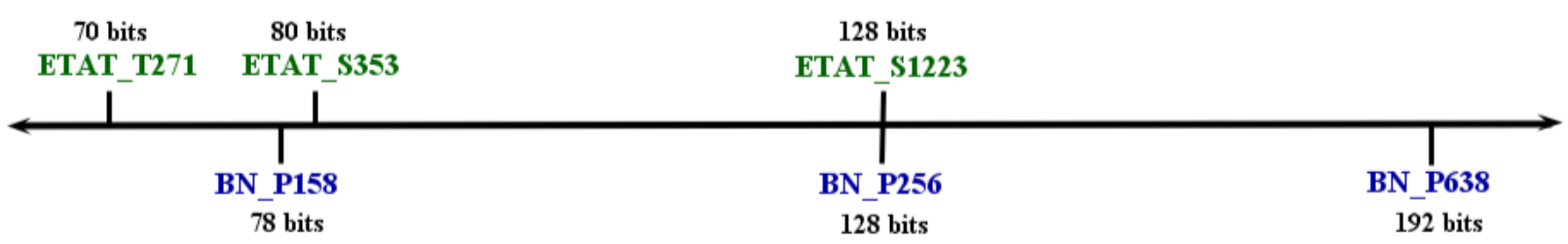

Figura 6.2: Nível de segurança das curvas elípticas escolhidas.

As curvas binárias selecionadas possuem grau de mergulho 4, e estão definidas sobre corpos binários de 271, 353 e 1223 bits. Estas curvas trabalham com emparelhamento simétrico.

No caso das curvas primas, foram selecionadas curvas elípticas conhecidas como BN (Barreto e Naehrig , 2006), definidas sobre corpos primos de 158, 256 e 638 bits. Estas curvas trabalham com emparelhamento assimétrico e possuem grau de mergulho 12, permitindo assim o uso de corpos algébricos menores, contudo, mantendo elevado nível de segurança. Por este motivo, são mais eficientes quando comparadas com curvas binárias.

A biblioteca RELIC-toolkit permite utilizar dois backends aritméticos diferentes: EASY (backend aritmético escrito pelos desenvolvedores da RELIC-toolkit, menos eficiente), e GMP (GNU Multiple Precision Library ${ }^{2}$ ). A Tabela 6.1 mostra o desempenho das principais operações envolvidas nos

\footnotetext{
${ }^{2}$ Biblioteca para manipulação de números de tamanho arbitrário muito conhecida pela comunidade científica e eficiente computacionalmente.
} 
protocolos apresentados no Capítulo 5. Estas operações são: emparelhamento $(e()$,$) , exponenciação$ no grupo $\mathbb{G}_{T}\left(E_{\mathbb{G}_{T}}\right)$, multiplicação no grupo $\mathbb{G}_{T}\left(M_{\mathbb{G}_{T}}\right)$, multiplicação no grupo $\mathbb{G}_{1}\left(M_{\mathbb{G}_{1}}\right)$, adição no grupo $\mathbb{G}_{1}\left(A_{\mathbb{G}_{1}}\right)$, multiplicação no grupo $\mathbb{G}_{2}\left(M_{\mathbb{G}_{2}}\right)$, adição no grupo $\mathbb{G}_{2}\left(M_{\mathbb{G}_{2}}\right)$. Os emparelhamentos simétrico e assimétrico utilizados foram o $\eta T$ (Barreto et al., 2007) e o Optimal-Ate (Vercauteren, 2010), respectivamente.

Os testes foram realizados no servidor e nos três dispositivos de menor poder computacional, apresentados na Seção 6.1, utilizando as 6 curvas elípticas escolhidas e os backends aritméticos disponíveis. Cada valor apresentado é o resultado da média de 50 amostras. As versões utilizadas das bibliotecas RELIC-toolkit e GMP foram 0.3.1 e 5.0.5, respectivamente.

\begin{tabular}{|c|c|c|c|c|c|c|c|c|}
\hline Backend EASY & Dispositivo & $e()$, & $E_{\mathbb{G}_{T}}$ & $M_{\mathbb{G}_{T}}$ & $M_{\mathbb{G}_{1}}$ & $A_{\mathbb{G}_{1}}$ & $M_{\mathbb{G}_{2}}$ & $A_{\mathbb{G}_{2}}$ \\
\hline \multirow[t]{4}{*}{ ETAT_T271 } & $\mathrm{PC}$ & 8.1 & 9.5 & 0.07 & 4.9 & 0.04 & & \\
\hline & $\mathrm{RPi}$ & 85.4 & 99.0 & 0.75 & 50.0 & 0.44 & & \\
\hline & MM1 & 75.1 & 88.1 & 0.66 & 45.9 & 0.38 & & \\
\hline & GN7 & 53.3 & 62.9 & 0.46 & 32.6 & 0.27 & & \\
\hline \multirow[t]{4}{*}{ ETAT_S353 } & $\mathrm{PC}$ & 15.6 & 19.5 & 0.11 & 10.0 & 0.06 & & \\
\hline & $\mathrm{RPi}$ & 165.1 & 205.3 & 1.1 & 100.7 & 0.66 & & \\
\hline & MM1 & 144.1 & 184.3 & 0.98 & 90.5 & 0.58 & & \\
\hline & GN7 & 104.0 & 133.2 & 0.71 & 65.2 & 0.41 & & \\
\hline \multirow[t]{4}{*}{ ETAT_S1223 } & $\mathrm{PC}$ & 388.1 & 499.8 & 0.79 & 213.5 & 0.45 & & \\
\hline & $\mathrm{RPi}$ & 3970.1 & 5064.1 & 8.2 & 2190.6 & 4.6 & & \\
\hline & MM1 & 3394.7 & 4332.8 & 7.0 & 1897.4 & 4.0 & & \\
\hline & GN7 & 2467.8 & 3150.7 & 5.1 & 1371.6 & 2.9 & & \\
\hline \multirow[t]{4}{*}{$\mathrm{BN} \_\mathrm{P} 158$} & $\mathrm{PC}$ & 16.7 & 16.1 & 0.11 & 1.6 & 0.01 & 8.2 & 0.03 \\
\hline & $\mathrm{RPi}$ & 146.8 & 140.9 & 1.0 & 14.7 & 0.1 & 71.5 & 0.24 \\
\hline & MM1 & 164.6 & 157.9 & 1.0 & 17.0 & 0.1 & 80.6 & 0.25 \\
\hline & GN7 & 97.4 & 93.6 & 0.64 & 9.8 & 0.06 & 46.8 & 0.16 \\
\hline \multirow[t]{4}{*}{$\mathrm{BN} \_\mathrm{P} 256$} & PC & 54.5 & 52.3 & 0.23 & 5.5 & 0.02 & 28.4 & 0.06 \\
\hline & $\mathrm{RPi}$ & 457.3 & 437.4 & 1.9 & 46.7 & 0.19 & 238.9 & 0.48 \\
\hline & MM1 & 504.5 & 482.1 & 2.0 & 51.5 & 0.19 & 263.4 & 0.48 \\
\hline & GN7 & 295.1 & 283.9 & 1.2 & 30.5 & 0.12 & 152.9 & 0.3 \\
\hline \multirow[t]{4}{*}{$\mathrm{BN} \_\mathrm{P} 638$} & $\mathrm{PC}$ & 599.9 & 585.5 & 1.0 & 64.1 & 0.1 & 340.9 & 0.26 \\
\hline & $\mathrm{RPi}$ & 5218.6 & 5067.9 & 8.7 & 547.8 & 0.93 & 2980.9 & 2.3 \\
\hline & MM1 & 5190.2 & 5029.6 & 8.6 & 557.9 & 0.93 & 2968.3 & 2.3 \\
\hline & GN7 & 3262.9 & 3160.8 & 5.4 & 350.3 & 0.58 & 1850.5 & 1.4 \\
\hline Backend GMP & Dispositivo & $e()$, & $E_{\mathbb{G}_{T}}$ & $M_{\mathbb{G}_{T}}$ & $M_{\mathbb{G}_{1}}$ & $A_{\mathbb{G}_{1}}$ & $M_{\mathbb{G}_{2}}$ & $A_{\mathbb{G}_{2}}$ \\
\hline \multirow[t]{4}{*}{ ETAT_T271 } & $\mathrm{PC}$ & 5.1 & 6.0 & 0.04 & 3.5 & 0.03 & & \\
\hline & $\mathrm{RPi}$ & 45.5 & 53.0 & 0.38 & 31.0 & 0.25 & & \\
\hline & MM1 & 42.4 & 50.6 & 0.36 & 30.6 & 0.22 & & \\
\hline & GN7 & 16.5 & 19.7 & 0.14 & 11.6 & 0.09 & & \\
\hline \multirow[t]{4}{*}{ ETAT_S353 } & $\mathrm{PC}$ & 8.6 & 10.7 & 0.06 & 6.1 & 0.04 & & \\
\hline & $\mathrm{RPi}$ & 79.5 & 99.7 & 0.52 & 56.3 & 0.32 & & \\
\hline & MM1 & 73.2 & 94.9 & 0.49 & 54.0 & 0.3 & & \\
\hline & GN7 & 51.9 & 67.3 & 0.34 & 38.0 & 0.21 & & \\
\hline \multirow[t]{4}{*}{ ETAT_S1223 } & $\mathrm{PC}$ & 117.1 & 150.9 & 0.23 & 75.3 & 0.13 & & \\
\hline & $\mathrm{RPi}$ & 1229.4 & 1581.9 & 2.4 & 783.7 & 1.4 & & \\
\hline & MM1 & 1285.8 & 1653.9 & 2.7 & 833.4 & 1.7 & & \\
\hline & GN7 & 479.7 & 617.5 & 0.96 & 299.4 & 0.56 & & \\
\hline \multirow[t]{4}{*}{ BN_P158 } & $\mathrm{PC}$ & 5.5 & 5.3 & 0.04 & 0.56 & 0.004 & 2.4 & 0.01 \\
\hline & $\mathrm{RPi}$ & 42.3 & 41.2 & 0.27 & 4.5 & 0.03 & 17.1 & 0.07 \\
\hline & MM1 & 50.0 & 48.9 & 0.27 & 5.2 & 0.04 & 20.3 & 0.07 \\
\hline & GN7 & 31.6 & 30.9 & 0.18 & 3.5 & 0.02 & 12.9 & 0.05 \\
\hline \multirow[t]{4}{*}{$\mathrm{BN} \_\mathrm{P} 256$} & $\mathrm{PC}$ & 13.1 & 12.7 & 0.05 & 1.4 & 0.01 & 6.1 & 0.01 \\
\hline & $\mathrm{RPi}$ & 94.7 & 91.6 & 0.38 & 10.2 & 0.04 & 41.5 & 0.1 \\
\hline & MM1 & 111.6 & 109.0 & 0.5 & 11.4 & 0.05 & 50.0 & 0.1 \\
\hline & GN7 & 68.2 & 66.8 & 0.25 & 7.6 & 0.03 & 29.7 & 0.06 \\
\hline \multirow[t]{4}{*}{ BN_P638 } & $\mathrm{PC}$ & 90.1 & 88.4 & 0.15 & 10.0 & 0.02 & 47.5 & 0.04 \\
\hline & $\mathrm{RPi}$ & 624.9 & 614.6 & 1.0 & 70.5 & 0.12 & 318.0 & 0.27 \\
\hline & MM1 & 690.5 & 679.2 & 1.1 & 78.8 & 0.21 & 351.6 & 0.32 \\
\hline & GN7 & 408.6 & 403.6 & 0.66 & 46.0 & 0.07 & 207.0 & 0.17 \\
\hline
\end{tabular}

Tabela 6.1: Tempo de execução das operações (em milissegundos), em diferentes dispositivos e níveis de segurança.

No Apêndice B, apresentamos a Tabela B.3, com os intervalos de confiança dos valores obtidos neste experimento. Nas Figuras 6.3 e 6.4 são exibidos dois gráficos comparando os resultados da Tabela 6.1. 
ETAT_T271 (PC)
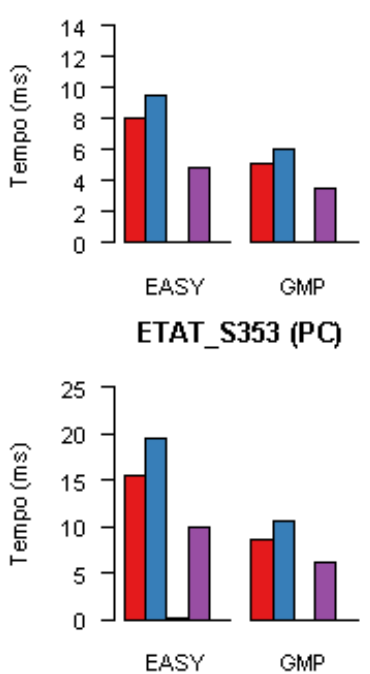

ETAT_S1223 (PC)

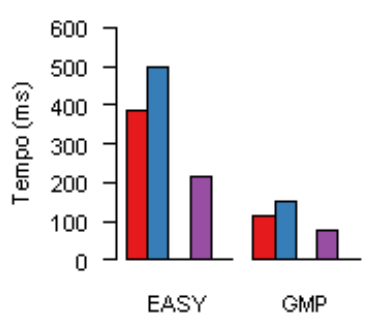

ETAT_T271 (RPi)
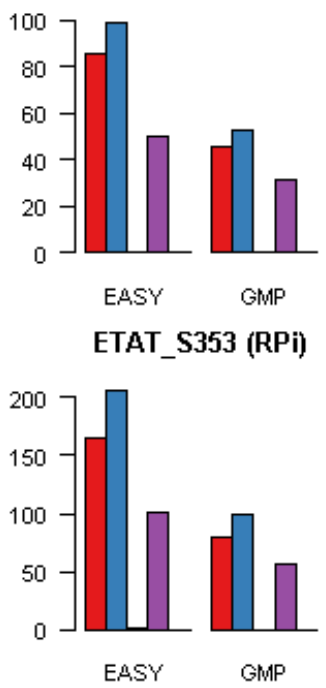

ETAT_S1223 (RPi)

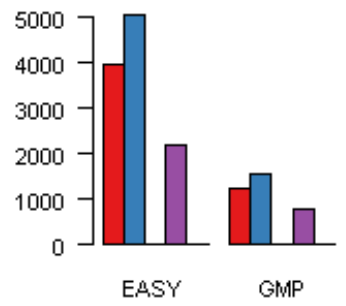

ETAT_T271 (MHA1)
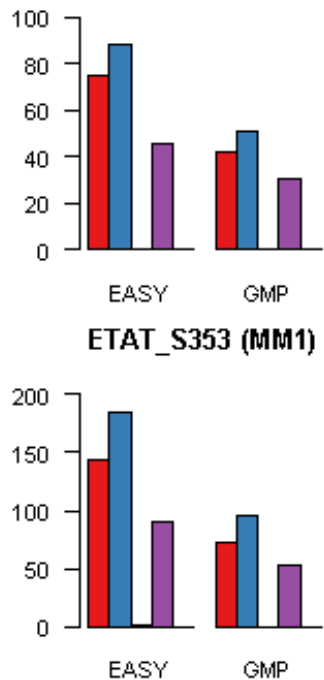

ETAT_S1223 (MH1)

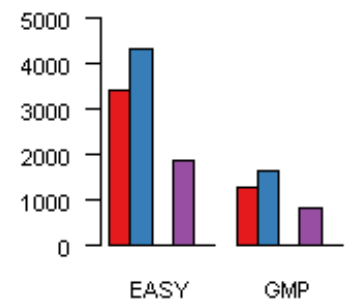

ETAT_T271 (GN7)
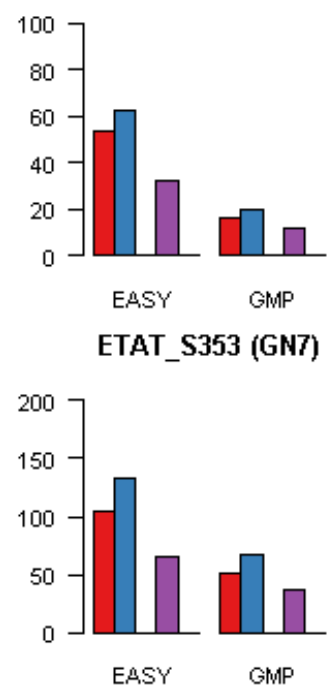

ETAT_S1223 (GN7)

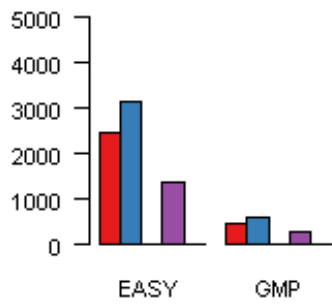

Figura 6.3: Comparação entre os tempos de cada operação em curvas binárias (em milissegundos). 


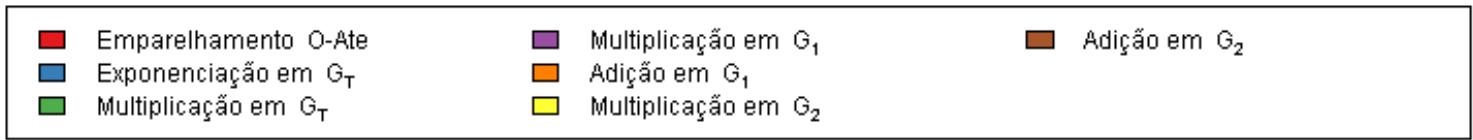

BN_P158 (PC)

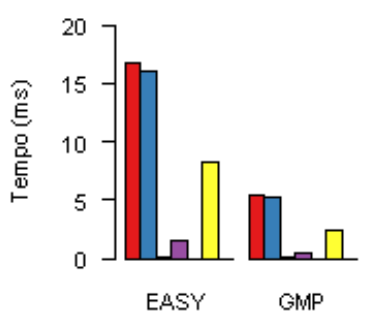

BN_P256 (PC)
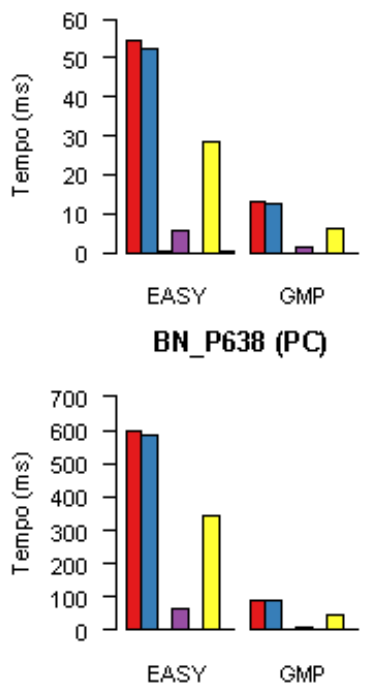

BN_P158 (RPi)

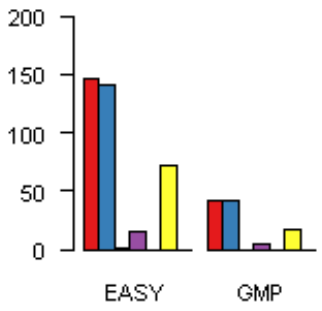

BN_P256 (RPi)
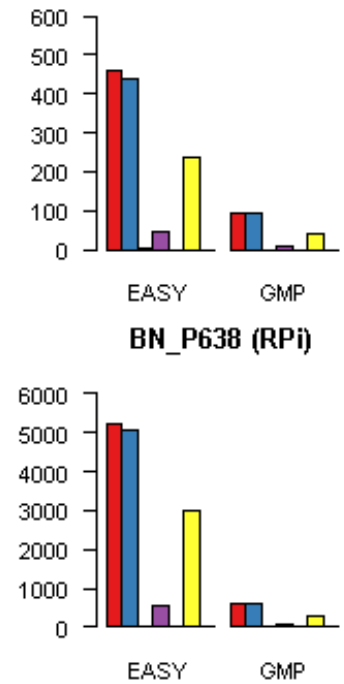

BN_P158 (MM1)

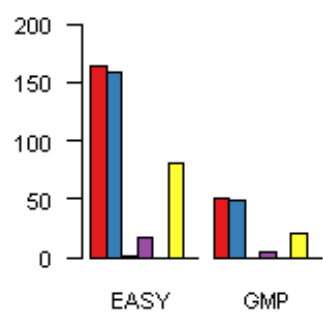

BN_P256 (MM1)
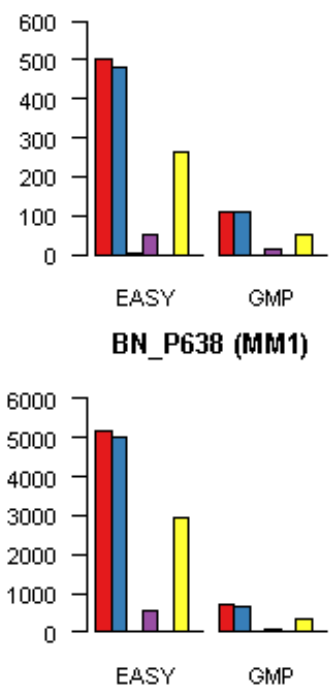

BN_P158 (GN7)

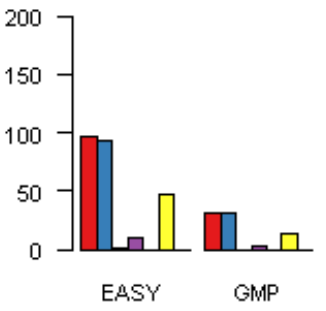

BN_P256 (GN7)
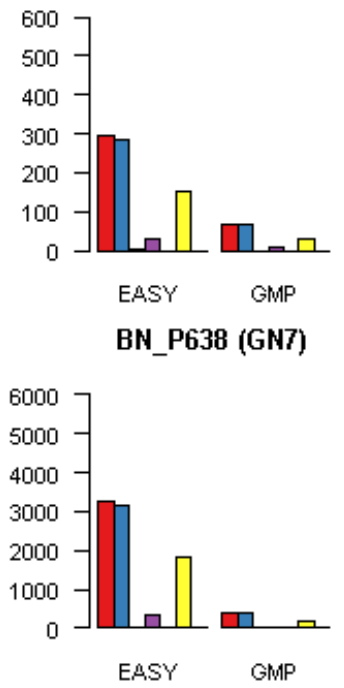

Figura 6.4: Comparação entre os tempos de cada operação em curvas primas (em milissegundos).

A partir dos dados obtidos, podemos verificar que o backend aritmético GMP é realmente muito mais eficiente que o backend EASY, e portanto deve ser utilizado sempre que possível. Outra informação importante é que o desempenho das operações em curvas primas é melhor que em curvas binárias, o que ocorre devido ao tamanho menor dos grupos algébricos envolvidos.

Podemos afirmar também que as operações de maior peso são: emparelhamento e exponenciação em $\mathbb{G}_{T}$ (devendo sempre serem evitadas), seguidas das operações de multiplicação escalar nos grupos $\mathbb{G}_{2}$ e $\mathbb{G}_{1}$. Em especial, um truque para evitar a exponenciação em $\mathbb{G}_{T}$ é utilizar a propriedade de bilinearidade dos emparelhamentos (apresentada no Capítulo 3). Assim, é possível converter operações de exponenciação no grupo $\mathbb{G}_{T}$ em multiplicações nos grupos $\mathbb{G}_{1}$ ou $\mathbb{G}_{2}$, resultando em um ganho de eficiência maior que $50 \%$ para este tipo de conversão.

\subsection{Adaptação eficiente para emparelhamento assimétrico}

Outra forma de implementar protocolos mais eficientes é distribuir os valores dos grupos $\mathbb{G}_{1}$ e $\mathbb{G}_{2}$ de forma que o dispositivo com menor poder computacional (cliente) realize mais cálculos no grupo $\mathbb{G}_{1}$, enquanto o servidor (geralmente com maior poder computacional) realiza mais cálculos no grupo $\mathbb{G}_{2}$. Como visto na Tabela 6.1 , operações em $\mathbb{G}_{2}$ chegam a custar em média quarto vezes mais que operações em $\mathbb{G}_{1}$. Por isso devemos maximizar a quantidade de multiplicações em $\mathbb{G}_{1}$ no lado do cliente.

Apresentamos, como exemplo, duas adaptações do protocolo Huang-Cao (Huang e Cao, 2009) para emparelhamento assimétrico. A Figura 6.5 ilustra o caso menos eficiente para o cliente (repre- 
sentado pelo usuário $B$ ), e a Figura 6.6 o caso mais eficiente. Considere as adaptações discutidas na Seção 5.4.

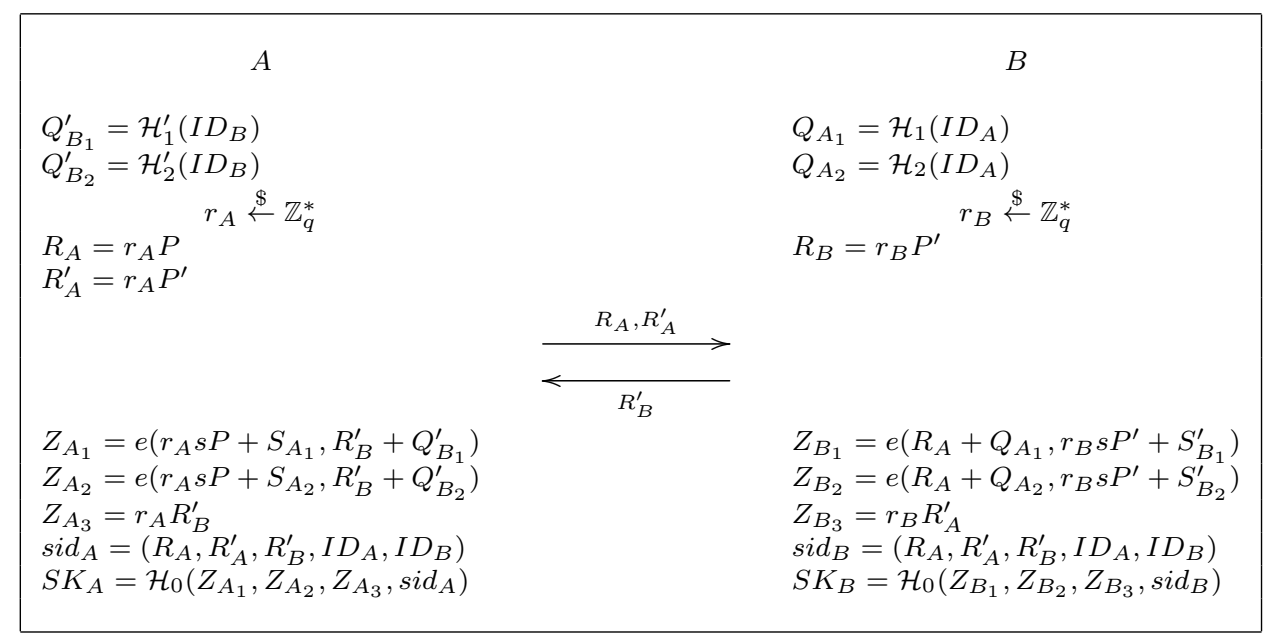

Figura 6.5: Protocolo Huang-Cao assimétrico - caso 1

Note que no cenário da Figura 6.5, a partir do momento da geração dos segredos temporários, o servidor (representado pelo usuário $A$ ) terá que executar duas multiplicações em $\mathbb{G}_{1}$ e duas multiplicações em $\mathbb{G}_{2}$. Já o cliente, com menor poder computacional, executará três multiplicações em $\mathbb{G}_{2}$, que são mais custosas. Portanto, neste cenário, o custo computacional para o cliente é maior do que para o servidor.

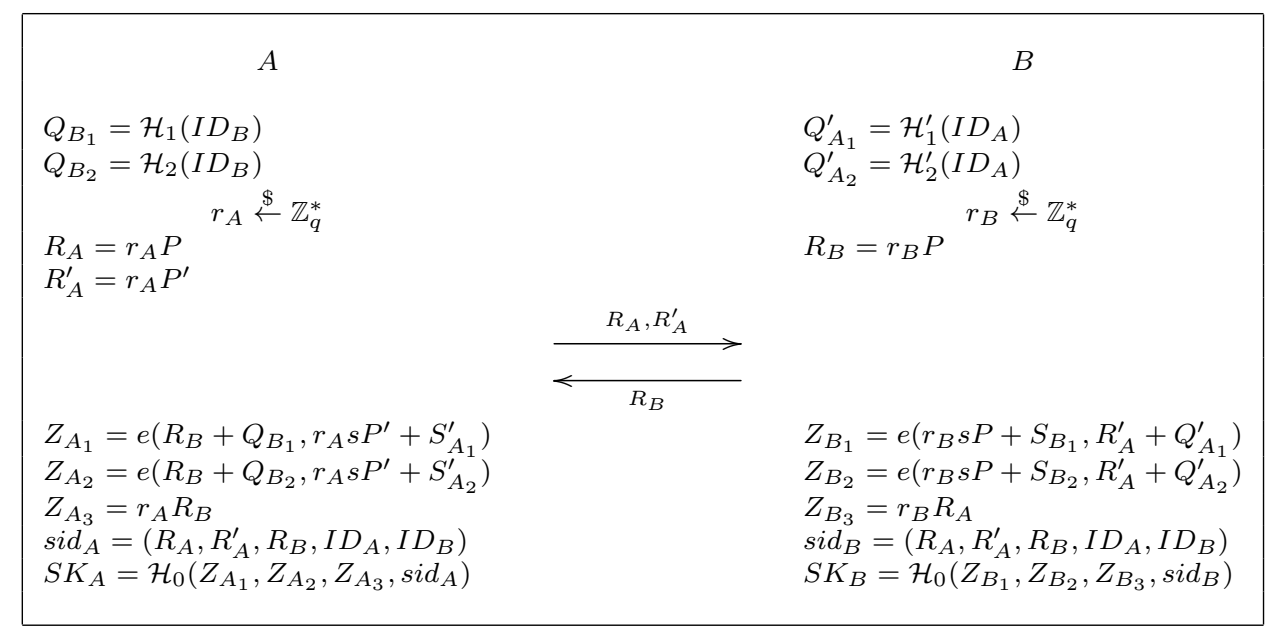

Figura 6.6: Protocolo Huang-Cao assimétrico - caso 2

O cenário da Figura 6.6 é o mais vantajoso para o cliente, que necessita calcular apenas três multiplicações em $\mathbb{G}_{1}$. Já o servidor calcula duas multiplicações em $\mathbb{G}_{1}$ e duas multiplicações em $\mathbb{G}_{2}$. Adaptações similares a esta foram produzidas para todos os protocolos estudados, com o objetivo de tornar o código utilizado pelos dispositivos (no papel de clientes) o mais eficiente possível.

\subsection{Comparação de desempenho entre os protocolos analisados}

Nesta seção comparamos o desempenho dos protocolos apresentados no Capítulo 5, nos diferentes dispositivos apresentados na Seção 6.1. A nomenclatura adotada refere-se às iniciais dos autores, e ao problema computacional sob o qual está definido o protocolo, conforme detalhado a seguir:

- Protocolos no modelo baseado em identidade: 
- HC-BDH: Huang e Cao (2009)

- HLZ-GBDH: Hu et al. (2009)

- CC-BDH: Chow e Choo (2007)

- NCLH-BDH e NCLH-GBDH: Ni et al. (2011)

- NCL-BDH: Ni et al. (2012)

- Protocolos no modelo sem certificado:

- LBG-BDH e LBG-GBDH: Lippold et al. (2009)

- GOT-BDH e GOT-GBDH: Goya et al. (2010)

- GNT3-BDH: Goya (2011)

- GNT4-BDH: Goya (2011)

- GNT1-GBDH: Goya et al. (2011)

- GNT2-GBDH: Goya (2011)

Para facilitar a visualização de quais protocolos estão relacionados a determinado modelo de segurança (introduzidos na Seção 4.4.2), apresentamos as Tabelas 6.2 (com protocolos no modelo de criptografia baseado em identidade) e 6.3 (com protocolos no modelo de criptografia sem certificado). A primeira linha representa o modelo de segurança mais forte, enquanto que a última linha representa o modelo de segurança mais fraco.

\begin{tabular}{lcc} 
& \multicolumn{2}{c}{ Problema Computacional } \\
\hline Modelo de segurança & BDH & GBDH \\
\hline eCK & HC, NCLH NCL $^{*}$ & HLZ, NCLH \\
CK & CC & \\
\hline
\end{tabular}

Tabela 6.2: Relação entre protocolos no modelo baseado em identidade e modelos de segurança.

* Protocolos com modo escrow.

\begin{tabular}{lcc} 
& \multicolumn{2}{c}{ Problema Computacional } \\
\hline Modelo de segurança & BDH & GBDH \\
\hline Mal-LBG & & GNT1 \\
LBG & LBG, GOT, GNT3 & LBG, GOT \\
Mal-SJ $^{+}$ & GNT4 & GNT2 \\
SJ $^{+}$ & \\
\hline
\end{tabular}

Tabela 6.3: Relação entre protocolos no modelo sem certificado e modelos de segurança.

A Tabela 6.4 refere-se à quantidade de operações quando utilizadas curvas binárias (emparelhamento simétrico), e a Tabela 6.5 refere-se à quantidade de operações quando utilizadas curvas primas (emparelhamento assimétrico). Ambas apresentam a quantidade de operações que, de fato, são realizadas pelo cliente para cada protocolo, nos códigos-fonte criados. Foram tomados certos cuidados para que fosse possível obter uma implementação eficiente, dentre os quais podemos citar: o aproveitamento de valores já pré-calculados anteriormente, substituição das operações de exponenciação em $\mathbb{G}_{T}$ por multiplicações em $\mathbb{G}$ (quando a troca é de um para um), e prioridade de operações no grupo $\mathbb{G}_{1}$ no lado do cliente (quando utilizado emparelhamento assimétrico).

O cenário sem pré-computação representa os protocolos da maneira como são definidos. Já o cenário com précomputação é considerado quando um usuário $A$ se comunica frequentemente com um usuário $B$. Assim, alguns valores referentes a $B$ não precisam ser recalculados a cada nova sessão. 


\begin{tabular}{|c|c|c|c|c|c|c|}
\hline Protocolos ID-Based & Pré-computação & $e()$, & $E_{\mathbb{G}_{T}}$ & $M_{\mathbb{G}_{T}}$ & $M_{\mathbb{G}}$ & $A_{\mathbb{G}}$ \\
\hline \multirow{2}{*}{$\mathrm{HC}-\mathrm{BDH}$} & Não & 2 & 0 & 0 & 3 & 4 \\
\hline & Sim & 2 & 0 & 0 & 3 & 4 \\
\hline \multirow[t]{2}{*}{ HLZ-GBDH } & Não & 2 & 0 & 0 & 2 & 2 \\
\hline & Sim & 2 & 0 & 0 & 2 & 2 \\
\hline \multirow{2}{*}{$\mathrm{CC}-\mathrm{BDH}$} & Não & 1 & 0 & 0 & 5 & 1 \\
\hline & Sim & 1 & 0 & 0 & 5 & 1 \\
\hline \multirow[t]{2}{*}{ NCLH-BDH } & Não & 6 & 2 & 2 & 2 & 0 \\
\hline & Sim & 2 & 2 & 2 & 2 & 0 \\
\hline \multirow[t]{2}{*}{ NCLH-GBDH } & Não & 3 & 1 & 1 & 2 & 0 \\
\hline & Sim & 1 & 1 & 1 & 2 & 0 \\
\hline \multirow[t]{2}{*}{ NCL-BDH } & Não & 2 & 0 & 0 & 2 & 4 \\
\hline & Sim & 2 & 0 & 0 & 2 & 4 \\
\hline Protocolos Certificateless & Pré-computação & $e()$, & $E_{\mathbb{G}_{T}}$ & $M_{\mathbb{G}_{T}}$ & $M_{\mathbb{G}}$ & $A_{\mathbb{G}}$ \\
\hline \multirow[t]{2}{*}{ LBG-BDH } & Não & 10 & 0 & 4 & 8 & 0 \\
\hline & Sim & 4 & 0 & 2 & 6 & 0 \\
\hline \multirow[t]{2}{*}{ GOT-BDH } & Não & 8 & 0 & 2 & 8 & 4 \\
\hline & Sim & 2 & 0 & 0 & 6 & 4 \\
\hline \multirow[t]{2}{*}{ GNT3-BDH } & Não & 6 & 0 & 2 & 8 & 4 \\
\hline & Sim & 2 & 0 & 0 & 6 & 4 \\
\hline \multirow[t]{2}{*}{ GNT4-BDH } & Não & 4 & 0 & 0 & 8 & 8 \\
\hline & Sim & 2 & 0 & 0 & 6 & 4 \\
\hline \multirow[t]{2}{*}{ GNT1-GBDH } & Não & 4 & 0 & 1 & 8 & 4 \\
\hline & Sim & 2 & 0 & 0 & 6 & 4 \\
\hline \multirow[t]{2}{*}{ LBG-GBDH } & Não & 5 & 0 & 2 & 8 & 0 \\
\hline & Sim & 2 & 0 & 1 & 6 & 0 \\
\hline \multirow[t]{2}{*}{ GOT-GBDH } & Não & 4 & 0 & 1 & 8 & 2 \\
\hline & Sim & 1 & 0 & 0 & 6 & 2 \\
\hline \multirow[t]{2}{*}{ GNT2-GBDH } & Não & 3 & 0 & 0 & 8 & 4 \\
\hline & Sim & 1 & 0 & 0 & 6 & 2 \\
\hline
\end{tabular}

Tabela 6.4: Quantidade de operações nos protocolos implementados (emparelhamento simétrico).

\begin{tabular}{|c|c|c|c|c|c|c|c|c|}
\hline Protocolos ID-Based & Pré-computação & $e()$, & $E_{\mathbb{G}_{T}}$ & $M_{\mathbb{G}_{T}}$ & $M_{\mathbb{G}_{1}}$ & $A_{\mathbb{G}_{1}}$ & $M_{\mathbb{G}_{2}}$ & $A_{\mathbb{G}_{2}}$ \\
\hline \multirow{2}{*}{$\mathrm{HC}-\mathrm{BDH}$} & Não & 2 & 0 & 0 & 3 & 2 & 0 & 2 \\
\hline & Sim & 2 & 0 & 0 & 3 & 2 & 0 & 2 \\
\hline \multirow{2}{*}{ HLZ-GBDH } & Não & 2 & 0 & 0 & 2 & 1 & 0 & 1 \\
\hline & Sim & 2 & 0 & 0 & 2 & 1 & 0 & 1 \\
\hline \multirow[t]{2}{*}{$\mathrm{CC}-\mathrm{BDH}$} & Não & 1 & 0 & 0 & 4 & 0 & 1 & 1 \\
\hline & Sim & 1 & 0 & 0 & 4 & 0 & 1 & 1 \\
\hline \multirow[t]{2}{*}{ NCLH-BDH } & Não & 6 & 2 & 2 & 2 & 0 & 0 & 0 \\
\hline & Sim & 2 & 2 & 2 & 2 & 0 & 0 & 0 \\
\hline \multirow[t]{2}{*}{ NCLH-GBDH } & Não & 3 & 1 & 1 & 2 & 0 & 0 & 0 \\
\hline & Sim & 1 & 1 & 1 & 2 & 0 & 0 & 0 \\
\hline \multirow[t]{2}{*}{ NCL-BDH } & Não & 2 & 0 & 0 & 2 & 2 & 0 & 2 \\
\hline & Sim & 2 & 0 & 0 & 2 & 2 & 0 & 2 \\
\hline Protocolos Certificateless & Pré-computação & $e()$, & $E_{\mathbb{G}_{T}}$ & $M_{\mathbb{G}_{T}}$ & $M_{\mathbb{G}_{1}}$ & $A_{\mathbb{G}_{1}}$ & $M_{\mathbb{G}_{2}}$ & $A_{\mathbb{G}_{2}}$ \\
\hline \multirow[t]{2}{*}{ LBG-BDH } & Não & 10 & 0 & 4 & 8 & 0 & 0 & 0 \\
\hline & Sim & 4 & 0 & 2 & 6 & 0 & 0 & 0 \\
\hline \multirow[t]{2}{*}{ GOT-BDH } & Não & 8 & 0 & 2 & 8 & 2 & 0 & 2 \\
\hline & Sim & 2 & 0 & 0 & 6 & 2 & 0 & 2 \\
\hline \multirow{2}{*}{ GNT3-BDH } & Não & 6 & 0 & 2 & 8 & 2 & 0 & 2 \\
\hline & Sim & 2 & 0 & 0 & 6 & 2 & 0 & 2 \\
\hline \multirow[t]{2}{*}{ GNT4-BDH } & Não & 4 & 0 & 0 & 8 & 4 & 0 & 4 \\
\hline & Sim & 2 & 0 & 0 & 6 & 2 & 0 & 2 \\
\hline \multirow[t]{2}{*}{ GNT1-GBDH } & Não & 4 & 0 & 1 & 8 & 2 & 0 & 2 \\
\hline & Sim & 2 & 0 & 0 & 6 & 2 & 0 & 2 \\
\hline \multirow[t]{2}{*}{ LBG-GBDH } & Não & 5 & 0 & 2 & 8 & 0 & 0 & 0 \\
\hline & Sim & 2 & 0 & 1 & 6 & 0 & 0 & 0 \\
\hline \multirow[t]{2}{*}{ GOT-GBDH } & Não & 4 & 0 & 1 & 8 & 1 & 0 & 1 \\
\hline & Sim & 1 & 0 & 0 & 6 & 1 & 0 & 1 \\
\hline \multirow{2}{*}{ GNT2-GBDH } & Não & 3 & 0 & 0 & 8 & 2 & 0 & 2 \\
\hline & Sim & 1 & 0 & 0 & 6 & 1 & 0 & 1 \\
\hline
\end{tabular}

Tabela 6.5: Quantidade de operações nos protocolos implementados (emparelhamento assimétrico). 
As Tabelas 6.6 e 6.7 apresentam os tempos de execução ${ }^{3}$ dos protocolos estudados, nos três dispositivos analisados, em diferentes níveis de segurança. A primeira refere-se ao cenário sem pré-computação, e a segunda com pré-computação. A contagem de tempo iniciou-se no momento da escolha dos valores temporários, e encerrou-se após o cálculo da chave de sessão.

Para cada protocolo, em cada dispositivo, foram obtidas um total de 100 amostras de tempo, sendo 50 para o caso sem pré-computação, e 50 para o caso com pré-computação. Uma amostra corresponde a uma sessão completa, ou seja, escolhem-se novos valores temporários e calcula-se uma nova chave de sessão, diferente das anteriores. Cada um dos valores exibidos a seguir corresponde à média de 50 amostras.

Fica evidente na Tabela 6.6 que os protocolos cuja segurança se baseia na dificuldade do problema BDH (mais seguros) consomem mais tempo do que os que se baseiam no problema GBDH (menos seguro). Protocolos seguros sob modelos de segurança mais fracos também possuem um desempenho melhor, evidentemente.

\begin{tabular}{|c|c|c|c|c|c|c|c|}
\hline & & \multicolumn{6}{|c|}{ Curva Elíptica } \\
\hline Protocolos ID-Based & Dispositivo & ETAT_T271 & ETAT_S353 & $\mathrm{ETAT}_{\text {S }}$ S1223 & $\mathrm{BN}$ _ 158 & $\mathrm{BN}_{-} \mathrm{P} 256$ & $\mathrm{BN} \_\mathrm{P} 638$ \\
\hline \multirow[t]{3}{*}{$\mathrm{HC}-\mathrm{BDH}$} & $\mathrm{RPi}$ & $18 \overline{2.3}$ & $32 \overline{4.6}$ & $48 \overline{21.4}$ & $1 \overline{0} 4.1$ & $2 \overline{18.6}$ & $1 \overline{431.4}$ \\
\hline & MM1 & 179.5 & 329.9 & 4515.0 & 112.4 & 228.2 & 1424.5 \\
\hline & GN7 & 72.1 & 123.0 & 1885.8 & 49.0 & 93.9 & 529.0 \\
\hline \multirow[t]{3}{*}{ HLZ-GBDH } & RPi & 151.2 & 248.3 & 3010.7 & 94.8 & 206.4 & 1319.3 \\
\hline & MM1 & 147.4 & 243.5 & 2834.1 & 105.6 & 215.0 & 1355.9 \\
\hline & GN7 & 59.0 & 95.5 & 1188.3 & 45.8 & 87.6 & 484.0 \\
\hline \multirow[t]{3}{*}{$\mathrm{CC}-\mathrm{BDH}$} & RPi & 199.2 & 341.9 & 4539.2 & 80.9 & 175.4 & 1014.9 \\
\hline & MM1 & 200.6 & 342.7 & 4352.1 & 90.0 & 185.1 & 1096.4 \\
\hline & GN7 & 79.1 & 132.1 & 1765.4 & 37.6 & 75.5 & 382.1 \\
\hline \multirow[t]{3}{*}{ NCLH-BDH } & $\mathrm{RPi}$ & 442.4 & 782.3 & 12134.6 & 345.5 & 782.5 & 5044.6 \\
\hline & MM1 & 415.9 & 754.2 & 11254.2 & 376.9 & 785.0 & 5002.2 \\
\hline & GN7 & 164.6 & 288.1 & 4753.5 & 145.4 & 320.6 & 1822.1 \\
\hline \multirow[t]{3}{*}{ NCLH-GBDH } & $\mathrm{RPi}$ & 249.9 & 450.2 & 6826.2 & 177.7 & 403.2 & 2587.0 \\
\hline & MM1 & 243.8 & 429.8 & 6358.9 & 196.7 & 404.9 & 2558.2 \\
\hline & GN7 & 94.9 & 159.7 & 2680.6 & 76.2 & 163.4 & 937.2 \\
\hline \multirow{3}{*}{ NCL-BDH } & $\mathrm{RPi}$ & 151.6 & 271.1 & 4039.6 & 94.9 & 217.4 & 1364.6 \\
\hline & MM1 & 149.5 & 270.7 & 3775.3 & 106.5 & 215.5 & 1349.2 \\
\hline & GN7 & 60.7 & 104.5 & 1583.4 & 46.5 & 86.5 & 498.0 \\
\hline Protocolos Certificateless & Dispositivo & ETAT_T271 & ETAT_S353 & ETAT_S1223 & $\mathrm{BN} \_\mathrm{P} 158$ & $\mathrm{BN} \_\mathrm{P} 256$ & $\mathrm{BN} \_\mathrm{P} 638$ \\
\hline \multirow[t]{3}{*}{ LBG-BDH } & RPi & $67 \overline{8.5}$ & $118 \overline{7} .0$ & $177 \overline{18.0}$ & 465.0 & 1034.5 & 6817.1 \\
\hline & MM1 & 637.3 & 1129.0 & 16688.5 & 495.3 & 1068.9 & 6565.8 \\
\hline & GN7 & 257.0 & 429.3 & 6931.4 & 199.5 & 422.9 & 2421.8 \\
\hline \multirow[t]{3}{*}{ GOT-BDH } & $\mathrm{RPi}$ & 582.4 & 1033.6 & 15294.9 & 396.9 & 837.8 & 5637.1 \\
\hline & MM1 & 554.4 & 976.8 & 14269.0 & 398.5 & 880.2 & 5449.8 \\
\hline & GN7 & 213.9 & 375.5 & 5974.9 & 163.6 & 348.6 & 1975.5 \\
\hline \multirow[t]{3}{*}{ GNT3-BDH } & $\mathrm{RPi}$ & 494.4 & 871.4 & 12879.4 & 284.5 & 640.5 & 4307.6 \\
\hline & MM1 & 472.7 & 826.7 & 11981.9 & 313.7 & 675.3 & 4138.4 \\
\hline & GN7 & 183.3 & 320.2 & 5011.8 & 127.6 & 271.5 & 1526.0 \\
\hline \multirow[t]{3}{*}{ GNT4-BDH } & $\mathrm{RPi}$ & 402.2 & 712.6 & 10400.1 & 209.8 & 460.4 & 3010.7 \\
\hline & MM1 & 386.2 & 687.3 & 9710.1 & 227.2 & 480.3 & 2921.2 \\
\hline & GN7 & 151.1 & 267.1 & 4049.9 & 94.3 & 187.9 & 1083.9 \\
\hline \multirow[t]{3}{*}{ GNT1-GBDH } & RPi & 410.3 & 712.2 & 10381.9 & 215.4 & 451.5 & 3010.8 \\
\hline & MM1 & 384.7 & 687.8 & 9697.6 & 223.7 & 478.0 & 2920.5 \\
\hline & GN7 & 150.4 & 265.5 & 4054.1 & 92.7 & 188.5 & 1082.7 \\
\hline \multirow[t]{3}{*}{ LBG-GBDH } & RPi & 447.0 & 791.1 & 11592.3 & 243.3 & 563.2 & 3636.2 \\
\hline & MM1 & 426.0 & 751.6 & 10833.5 & 267.2 & 580.6 & 3531.1 \\
\hline & GN7 & 166.0 & 290.4 & 4524.6 & 111.9 & 232.0 & 1301.4 \\
\hline \multirow[t]{3}{*}{ GOT-GBDH } & $\mathrm{RPi}$ & 399.7 & 711.0 & 10366.7 & 207.8 & 468.9 & 2976.2 \\
\hline & MM1 & 383.7 & 677.2 & 9707.3 & 224.9 & 479.1 & 2925.9 \\
\hline & GN7 & 149.9 & 265.7 & 4043.9 & 93.9 & 187.8 & 1082.6 \\
\hline \multirow[t]{3}{*}{ GNT2-GBDH } & $\mathrm{RPi}$ & 354.1 & 633.9 & 9163.3 & 160.9 & 357.3 & 2367.7 \\
\hline & MM1 & 345.9 & 605.5 & 8564.5 & 184.6 & 379.7 & 2311.2 \\
\hline & GN7 & 133.9 & 230.7 & 3565.4 & 77.9 & 150.3 & 860.6 \\
\hline
\end{tabular}

Tabela 6.6: Tempos de execução dos protocolos (em milissegundos), sem pré-computação, para diferentes níveis de segurança.

Em curvas binárias, o protocolo HLZ-GBDH teve um desempenho médio entre $17 \%$ e $37 \%$ melhor que o protocolo HC-BDH. Já o protocolo CC-BDH chegou a ter um desempenho médio entre 3,9\% e 11,7\% pior que o protocolo HC-BDH nas curvas ETAT_T271 e ETAT_S353, e entre 3,6\% e 6,4\% melhor que o protocolo HC-BDH na curva ETAT_S1223. Já em curvas primas, ambos os protocolos HLZ-GBDH e CC-BDH apresentaram desempenho médio $6,5 \%$ e $22,2 \%$ melhor que o protocolo $\mathrm{HC}-\mathrm{BDH}$, respectivamente.

\footnotetext{
${ }^{3}$ Como os experimentos envolvem dois dispositivos se comunicando por rede TCP/IP, os resultados obtidos representam o tempo de parede.
} 
Entre os protocolos no modelo baseado em identidade com modo escrow, o protocolo NCL-BDH se destacou por ser mais eficiente que NCLH-BDH e NCLH-GBDH (mesmo este último estando sob a hipótese de um problema computacional mais fraco). Nos experimentos com curvas binárias, o protocolo NCL-BDH foi, em média, $65 \%$ e $38 \%$ mais eficiente que NCLH-BDH e NCLH-GBDH, nesta ordem. Em curvas primas a diferença foi ainda maior, sendo NCL-BDH $72 \%$ e $45 \%$, em média, mais eficiente do que NCLH-BDH e NCLH-GBDH, respectivamente.

No modelo sem certificado, de acordo com a Tabela 6.6, o protocolo GOT-BDH apresentou um desempenho médio 14\% melhor que o protocolo LBG-BDH em curvas binárias, e em torno de $18 \%$ em curvas primas. O protocolo GNT3-BDH foi em média $15 \%$ e $23 \%$ mais eficiente que o protocolo GOT-BDH, em curvas binárias e primas, respectivamente. Tal resultado era previsto, uma vez que GNT3-BDH é uma melhoria de GOT-BDH, que por sua vez é uma melhoria de LBG-BDH. Já o protocolo GNT4-BDH apresentou um desempenho médio $18 \%$ e $28 \%$ melhor que o protocolo GNT3-BDH, em curvas binárias e primas (nesta ordem), o que também era esperado visto que GNT4-BDH é demonstrado seguro sob o modelo de segurança $\mathrm{SJ}^{+}$(mais fraco).

Ainda no modelo sem certificado, o protocolo GOT-GBDH apresentou desempenho médio $10 \%$ melhor em relação ao protocolo LGB-GBDH em curvas binárias e $17 \%$ melhor em curvas primas, sendo ambos protocolos seguros sob o modelo de segurança LBG. Entretanto, o protocolo GNT1-GBDH destacou-se por ser mais eficiente que o protocolo LGB-GBDH, e ficar tecnicamente empatado com GOT-GBDH. Assim, recomenda-se sempre o uso do protocolo GNT1-GBDH no lugar de LBG-GBDH e GOT-GBDH, uma vez que é seguro sob o modelo de segurança Mal-LBG (mais forte que LBG). A única exceção é o protocolo GNT2-GBDH, seguro no modelo Mal-SJ ${ }^{+}$(mais fraco que Mal-LBG), e por este motivo mostrou-se, em média, $12 \%$ mais eficiente em curvas binárias, e $20 \%$ em curvas primas.

No Apêndice B, apresentamos a Tabela B.1, com os intervalos de confiança dos valores obtidos no cenário sem pré-computação. Nas Figuras 6.7 e 6.8 são exibidos gráficos comparando os resultados da Tabela 6.6.

\begin{tabular}{|llll|}
\hline$\square$ & $\mathrm{HC}-\mathrm{BDH}$ & $\square$ & NCLH-BDH \\
$\square$ & $\mathrm{HLZ}-\mathrm{GBDH}$ & $\square$ & NCLH-GBDH \\
$\square$ & $\mathrm{CC}-\mathrm{BDH}$ & $\square$ & NCL-BDH \\
\hline
\end{tabular}

\section{Raspberry Pi}
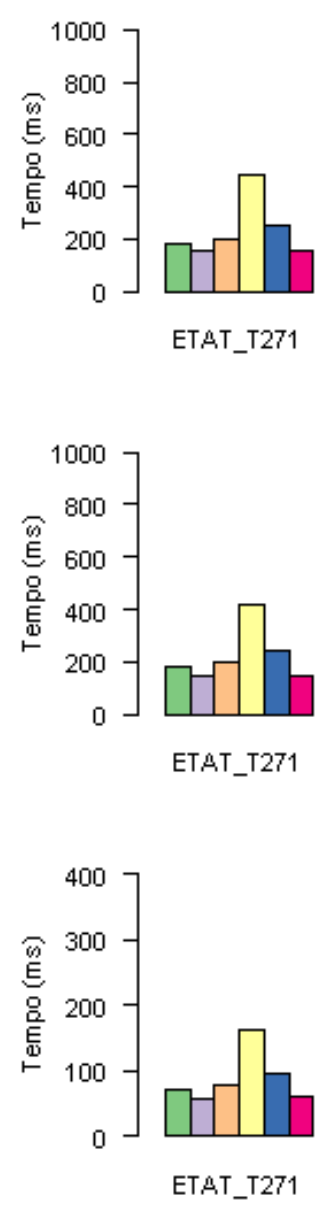

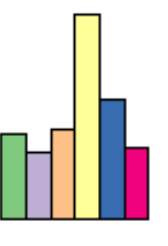

ETAT_S353

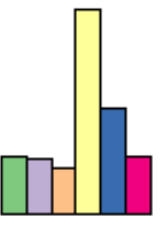

BN_P256

Motorola Milestone 1

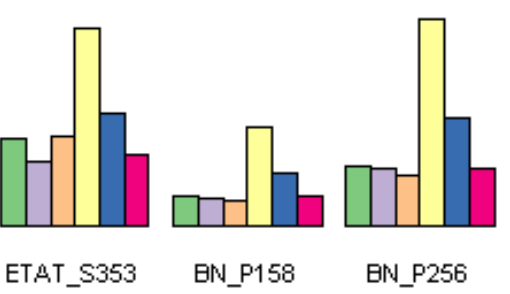

Google Nexus 7

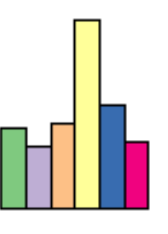

ETAT_S353

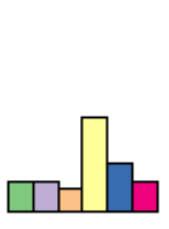

BN_P158

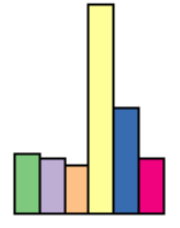

BN_P256
Raspberry Pi

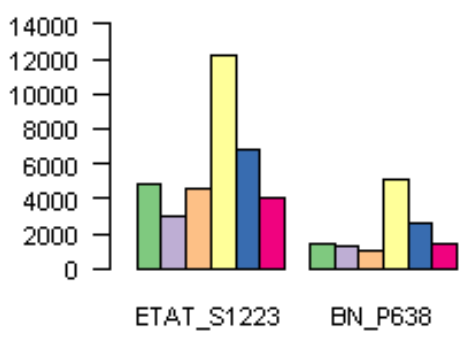

Motorola Milestone 1

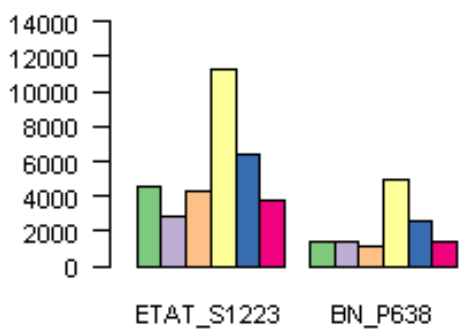

Google Nexus 7

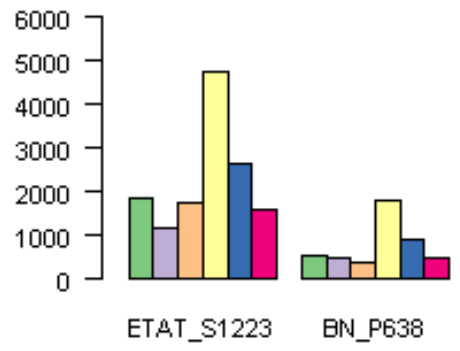

Figura 6.7: Comparação entre os protocolos baseados em identidade, em diferentes níveis de segurança, para o caso sem pré-computação. 


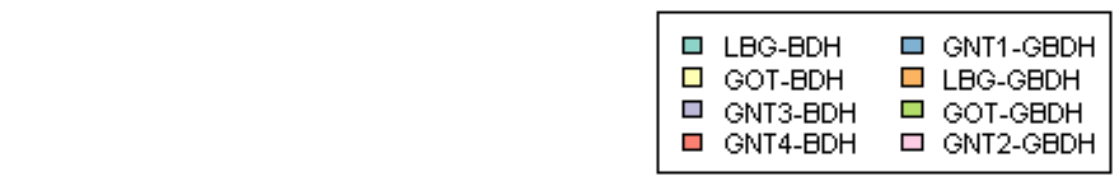

Raspberry Pi

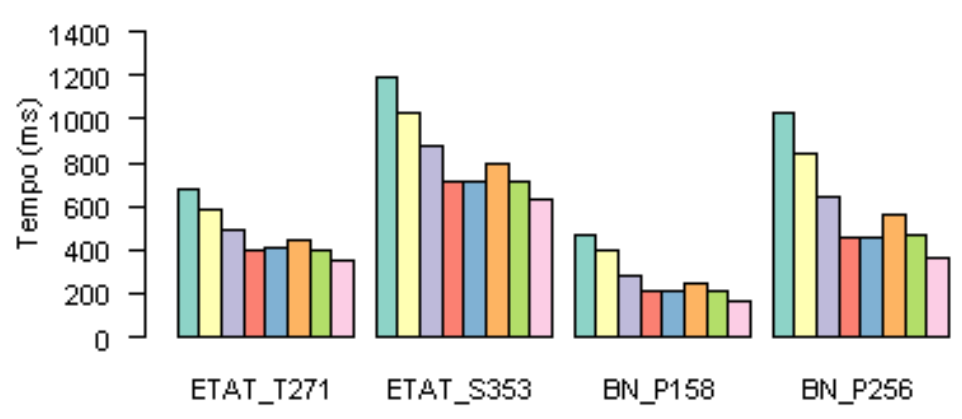

Motorola Milestone 1

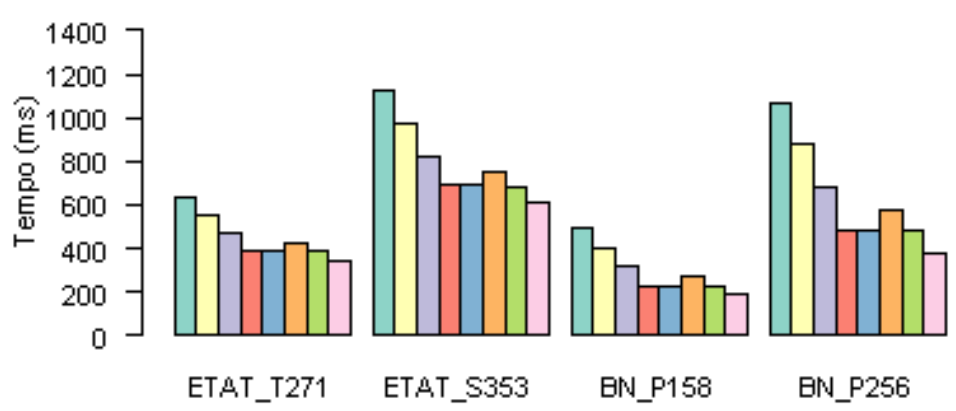

Google Nexus 7

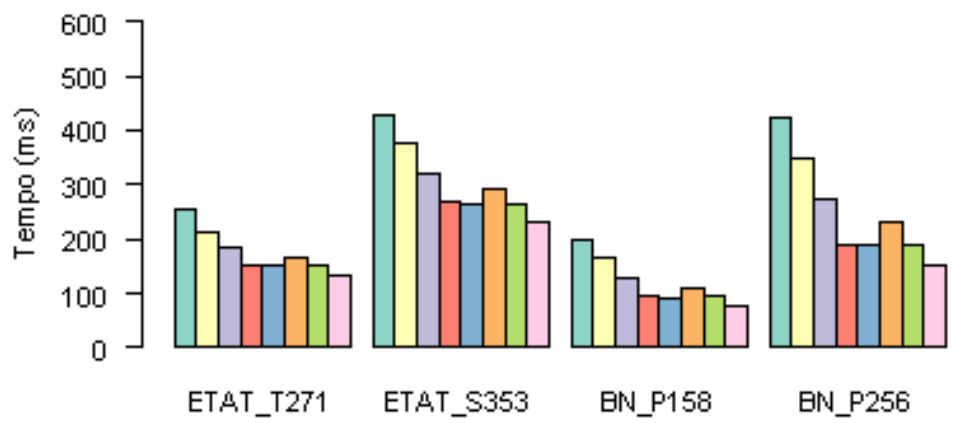

Raspberry Pi

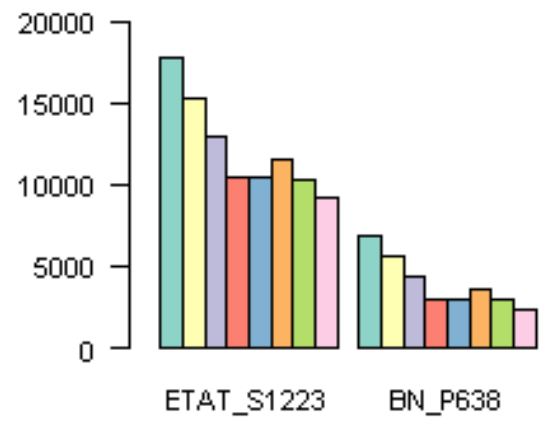

Motorola Milestone 1

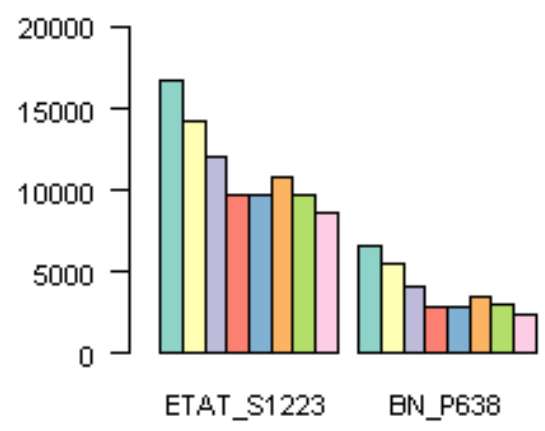

Google Nexus 7

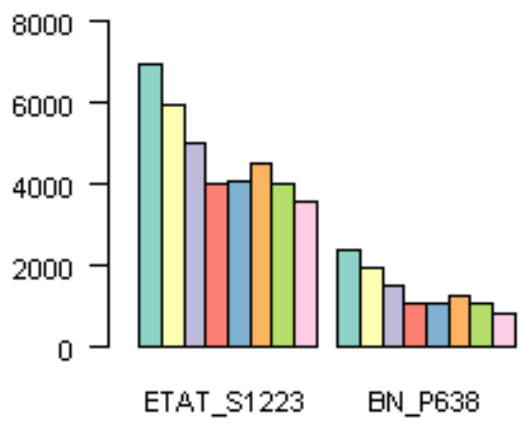

Figura 6.8: Comparação entre os protocolos sem certificado, em diferentes níveis de segurança, para o caso sem pré-computação.

A Tabela 6.7 apresenta o resultado dos experimentos no cenário com pré-computação. Pode-se observar que os tempos dos protocolos HC-BDH, HLZ-GBDH, CC-BDH e NCL-BDH são os mesmos observados na Tabela 6.6. Isto ocorre porque eles não possuem valores que possam ser armazenados entre diferentes sessões, uma vez que estes protocolos são mais simples, e todos os valores intermediários dependem dos segredos temporários (que mudam a cada nova sessão). Dentre os protocolos baseados em identidade estudados, os únicos que se beneficiam do cenário com pré-computação são NCLH-BDH e NCLH-GBDH. Apesar do empate técnico com NCLH-GBDH em curvas primas, o protocolo NCL-BDH continua mais vantajoso por estar sob a hipótese de um problema computacional mais forte, e seu uso é recomendado caso se opte por um protocolo com modo escrow. 


\begin{tabular}{|c|c|c|c|c|c|c|c|}
\hline \multirow[b]{2}{*}{ Protocolos ID-Based } & \multirow[b]{2}{*}{ Dispositivo } & \multicolumn{6}{|c|}{ Curva Elíptica } \\
\hline & & ETAT T271 & ETAT S353 & ETAT S1223 & BN $\quad$ P158 & BN $\quad$ P256 & $\begin{array}{ll}\text { BN } & \text { P638 }\end{array}$ \\
\hline \multirow[t]{3}{*}{ HC-BDH } & $\mathrm{RPi}$ & $18 \overline{\overline{2}} .3$ & $32 \overline{4.6}$ & $48 \overline{21} .4$ & 104.1 & $2 \overline{18} .6$ & $1 \overline{43} 1.4$ \\
\hline & MM1 & 179.5 & 329.9 & 4515.0 & 112.4 & 228.2 & 1424.5 \\
\hline & GN7 & 72.1 & 123.0 & 1885.8 & 49.0 & 93.9 & 529.0 \\
\hline \multirow[t]{3}{*}{ HLZ-GBDH } & $\mathrm{RPi}$ & 151.2 & 248.3 & 3010.7 & 94.8 & 206.4 & 1319.3 \\
\hline & MM1 & 147.4 & 243.5 & 2834.1 & 105.6 & 215.0 & 1355.9 \\
\hline & GN7 & 59.0 & 95.5 & 1188.3 & 45.8 & 87.6 & 484.0 \\
\hline \multirow[t]{3}{*}{ CC-BDH } & $\mathrm{RPi}$ & 199.2 & 341.9 & 4539.2 & 80.9 & 175.4 & 1014.9 \\
\hline & MM1 & 200.6 & 342.7 & 4352.1 & 90.0 & 185.1 & 1096.4 \\
\hline & GN7 & 79.1 & 132.1 & 1765.4 & 37.6 & 75.5 & 382.1 \\
\hline \multirow{3}{*}{ NCLH-BDH } & $\mathrm{RPi}$ & 258.7 & 467.2 & 7185.9 & 175.4 & 398.5 & 2573.9 \\
\hline & MM1 & 249.1 & 445.7 & 7210.7 & 195.9 & 403.0 & 2572.6 \\
\hline & GN7 & 98.4 & 169.9 & 2827.9 & 77.4 & 162.9 & 943.7 \\
\hline \multirow[t]{3}{*}{ NCLH-GBDH } & $\mathrm{RPi}$ & 158.1 & 291.1 & 4355.8 & 92.8 & 209.9 & 1346.3 \\
\hline & MM1 & 155.1 & 276.5 & 4109.1 & 105.9 & 214.8 & 1444.2 \\
\hline & GN7 & 62.8 & 105.5 & 1716.9 & 45.3 & 88.7 & 503.8 \\
\hline \multirow[t]{3}{*}{ NCL-BDH } & $\mathrm{RPi}$ & 151.6 & 271.1 & 4039.6 & 94.9 & 217.4 & 1364.6 \\
\hline & MM1 & 149.5 & 270.7 & 3775.3 & 106.5 & 215.5 & 1349.2 \\
\hline & GN7 & 60.7 & 104.5 & 1583.4 & 46.5 & 86.5 & 498.0 \\
\hline Protocolos Certificateless & Dispositivo & ETAT T271 & ETAT S353 & ETAT S1223 & BN $\quad \mathrm{P} 158$ & $\mathrm{BN} \quad \mathrm{P} 256$ & BN $\quad$ P638 \\
\hline \multirow[t]{3}{*}{ LBG-BDH } & $\mathrm{RPi}$ & $33 \overline{9} .4$ & $\overline{598.8}$ & $87 \overline{94.7}$ & $1 \overline{97.3}$ & $4 \overline{37.6}$ & $2 \overline{871.1}$ \\
\hline & MM1 & 324.3 & 570.7 & 8259.9 & 221.5 & 458.7 & 2791.1 \\
\hline & GN7 & 126.5 & 216.4 & 3437.5 & 89.1 & 179.6 & 1030.9 \\
\hline \multirow[t]{3}{*}{ GOT-BDH } & $\mathrm{RPi}$ & 246.5 & 443.1 & 6364.3 & 115.7 & 244.3 & 1630.8 \\
\hline & MM1 & 240.6 & 426.3 & 5973.0 & 127.4 & 276.2 & 1562.6 \\
\hline & GN7 & 94.8 & 160.1 & 2489.8 & 54.4 & 104.9 & 584.9 \\
\hline \multirow{3}{*}{ GNT3-BDH } & $\mathrm{RPi}$ & 248.2 & 441.6 & 6384.2 & 108.3 & 242.5 & 1613.5 \\
\hline & MM1 & 241.0 & 423.4 & 5960.3 & 125.5 & 263.3 & 1569.1 \\
\hline & GN7 & 94.6 & 160.2 & 2623.7 & 54.5 & 104.2 & 587.2 \\
\hline \multirow{3}{*}{ GNT4-BDH } & $\mathrm{RPi}$ & 247.3 & 440.4 & 6361.3 & 112.5 & 245.7 & 1601.6 \\
\hline & MM1 & 239.9 & 425.3 & 5950.7 & 128.9 & 261.8 & 1565.8 \\
\hline & GN7 & 94.6 & 160.7 & 2486.8 & 57.3 & 104.3 & 588.0 \\
\hline \multirow[t]{3}{*}{ GNT1-GBDH } & $\mathrm{RPi}$ & 253.3 & 441.7 & 6361.0 & 115.9 & 241.9 & 1603.0 \\
\hline & MM1 & 244.2 & 421.2 & 5944.5 & 128.3 & 261.5 & 1563.0 \\
\hline & GN7 & 94.4 & 160.4 & 2481.8 & 55.8 & 104.4 & 587.3 \\
\hline \multirow{3}{*}{ LBG-GBDH } & $\mathrm{RPi}$ & 246.9 & 440.0 & 6346.3 & 107.9 & 248.2 & 1598.1 \\
\hline & MM1 & 238.0 & 422.3 & 5952.7 & 129.7 & 264.6 & 1563.6 \\
\hline & GN7 & 93.8 & 159.3 & 2474.6 & 55.6 & 102.7 & 584.6 \\
\hline \multirow[t]{3}{*}{ GOT-GBDH } & $\mathrm{RPi}$ & 200.4 & 360.9 & 5124.1 & 67.7 & 151.3 & 959.0 \\
\hline & MM1 & 195.4 & 348.5 & 4840.6 & 82.3 & 163.9 & 990.5 \\
\hline & GN7 & 77.7 & 132.3 & 2001.4 & 39.3 & 66.1 & 363.5 \\
\hline \multirow{3}{*}{ GNT2-GBDH } & $\mathrm{RPi}$ & 200.1 & 362.0 & 5130.9 & 66.7 & 147.2 & 964.9 \\
\hline & MM1 & 196.8 & 347.1 & 4802.6 & 82.4 & 162.1 & 994.5 \\
\hline & GN7 & 77.8 & 133.0 & 2001.3 & 38.5 & 66.6 & 362.6 \\
\hline
\end{tabular}

Tabela 6.7: Tempos de execução dos protocolos (em milissegundos), com pré-computação, para diferentes niveis de segurança.

Os protocolos no modelo sem certificado analisados sofreram uma melhora significativa em desempenho no cenário com pré-computação, como observado na Tabela 6.7. Em geral, há um ganho médio de desempenho em torno de $38 \%$ à $70 \%$, quando comparamos os tempos dos mesmos protocolos com os da Tabela 6.6. Vale ressaltar que o protocolo GOT-BDH teve desempenho praticamente igual ao do GNT3-BDH, sendo este último mais recomendado pelo menor uso de memória RAM, de acordo com a Tabela 6.8. O protocolo GNT4-BDH também obteve desempenho semelhante ao dos protocolos GOT-BDH e GNT3-BDH, e seu uso neste cenário não é recomendado, visto que é demonstrado seguro sob modelo de segurança mais fraco que os outros dois.

Ainda de acordo com a Tabela 6.7, o protocolo GOT-GBDH foi, em média, $18 \%$ mais eficiente que GNT1GBDH em curvas binárias, e 37\% em curvas primas, uma diferença considerável quando comparamos com o cenário sem pré-computação. Neste caso, recomenda-se o uso de GOT-GBDH quando não houver preocupação com ataques originados pela autoridade do sistema.

No Apêndice B, apresentamos a Tabela B.2, com os intervalos de confiança dos valores obtidos no cenário com pré-computação. Nas Figuras 6.9 e 6.10 são exibidos gráficos comparando os resultados da Tabela 6.7. 

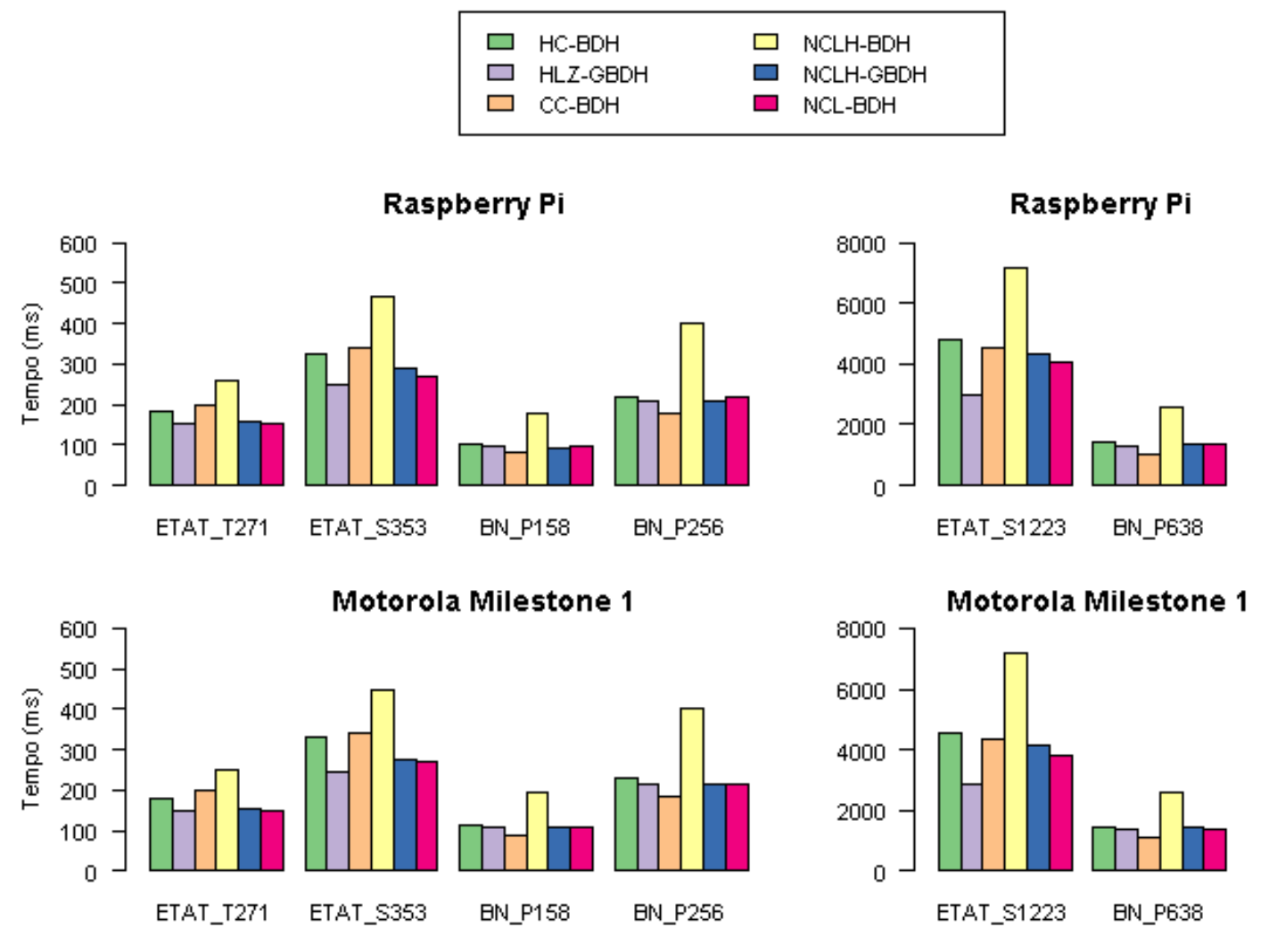

Google Nexus 7
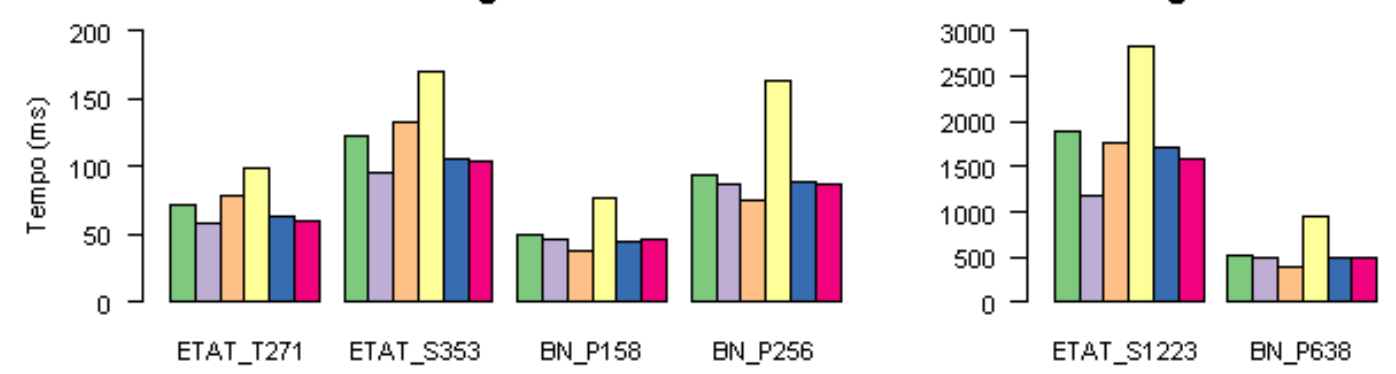

Figura 6.9: Comparação entre os protocolos baseados em identidade, em diferentes níveis de segurança, para o caso com pré-computação. 

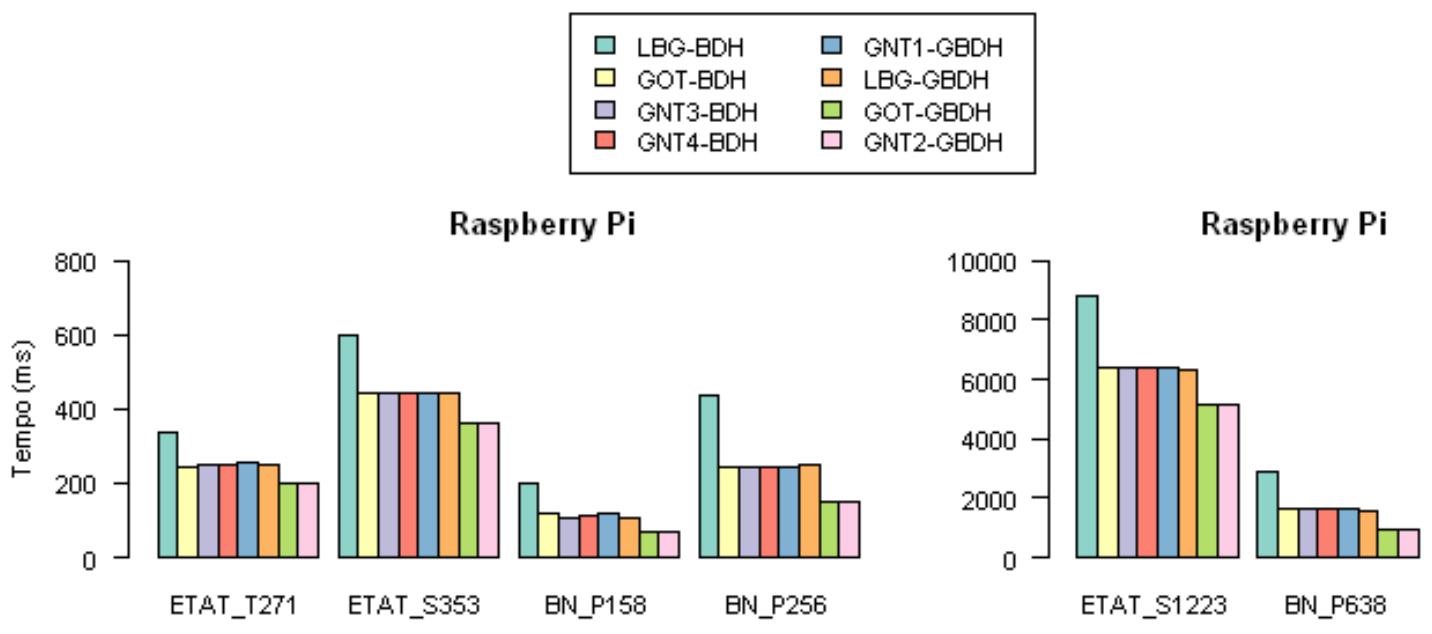

Motorola Milestone 1
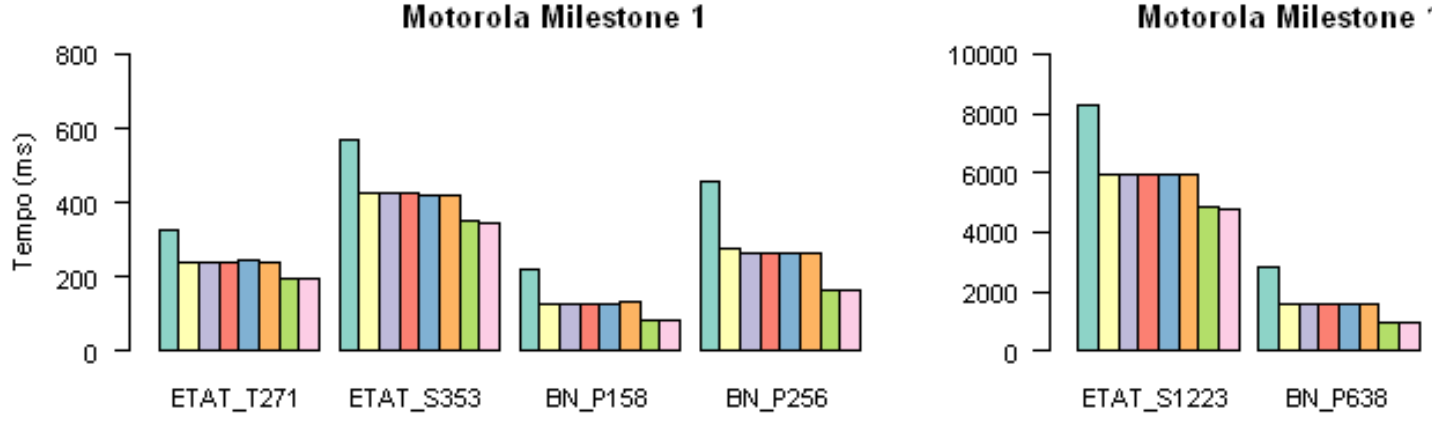

Google Nexus 7
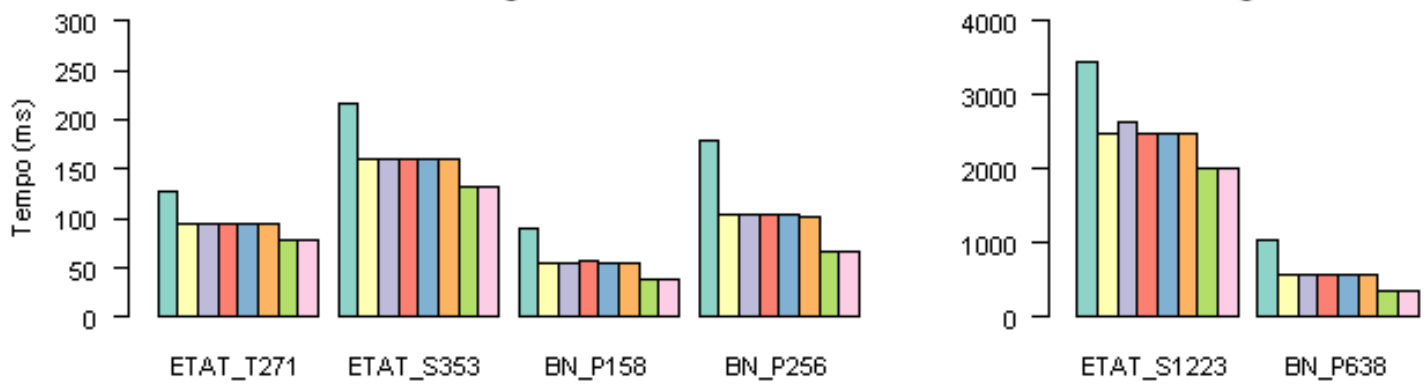

Figura 6.10: Comparação entre os protocolos sem certificado, em diferentes níveis de segurança, para o caso com pré-computação.

Nas Tabelas 6.8 e 6.9 apresentamos a quantidade de memória utilizada por cada protocolo e o comprimento das mensagens trocadas, respectivamente. A primeira é baseada na quantidade de variáveis utilizadas para implementar o protocolo e o tamanho do tipo de dado de cada uma delas. A segunda se baseia no comprimento dos tipos de dados utilizados para troca de mensagens. 


\begin{tabular}{lcccccc}
\cline { 2 - 7 } & \multicolumn{7}{c}{ Curva Elíptica } \\
\hline Protocolos ID-Based & ETAT_T271 & ETAT_S353 & ETAT_S1223 & BN_P158 & BN_P256 & BN_P638 \\
\hline HC-BDH & 1704 & 2244 & 7120 & 1624 & 2536 & 6184 \\
HLZ-GBDH & 1460 & 1916 & 6044 & 1376 & 2132 & 5156 \\
CC-BDH & 1612 & 2104 & 6572 & 1460 & 2228 & 5300 \\
NCLH-BDH & 2232 & 2952 & 9448 & 2812 & 4444 & 10972 \\
NCLH-GBDH & 1576 & 2080 & 6632 & 1904 & 2996 & 7364 \\
NCL-BDH & 1592 & 2096 & 6648 & 1496 & 2336 & 5696 \\
\hline Protocolos Certificateless & ETAT_T271 & ETAT_S353 & ETAT_S1223 & BN_P158 & BN_P256 & BN_P638 \\
\hline LBG-BDH & 2884 & 3808 & 12148 & 3324 & 5232 & 12864 \\
GOT-BDH & 2884 & 3808 & 12148 & 3448 & 5428 & 13348 \\
GNT3-BDH & 2596 & 3424 & 10900 & 2968 & 4660 & 11428 \\
GNT4-BDH & 2308 & 3040 & 9652 & 2488 & 3892 & 9508 \\
GNT1-GBDH & 2452 & 3232 & 10276 & 2728 & 4276 & 10468 \\
LBG-GBDH & 2228 & 2936 & 9332 & 2416 & 3784 & 9256 \\
GOT-GBDH & 2228 & 2936 & 9332 & 2540 & 3980 & 9740 \\
GNT2-GBDH & 1940 & 2552 & 8084 & 2060 & 3212 & 7820 \\
\hline
\end{tabular}

Tabela 6.8: Uso de memória (em bytes) dos protocolos analisados para diferentes níveis de segurança.

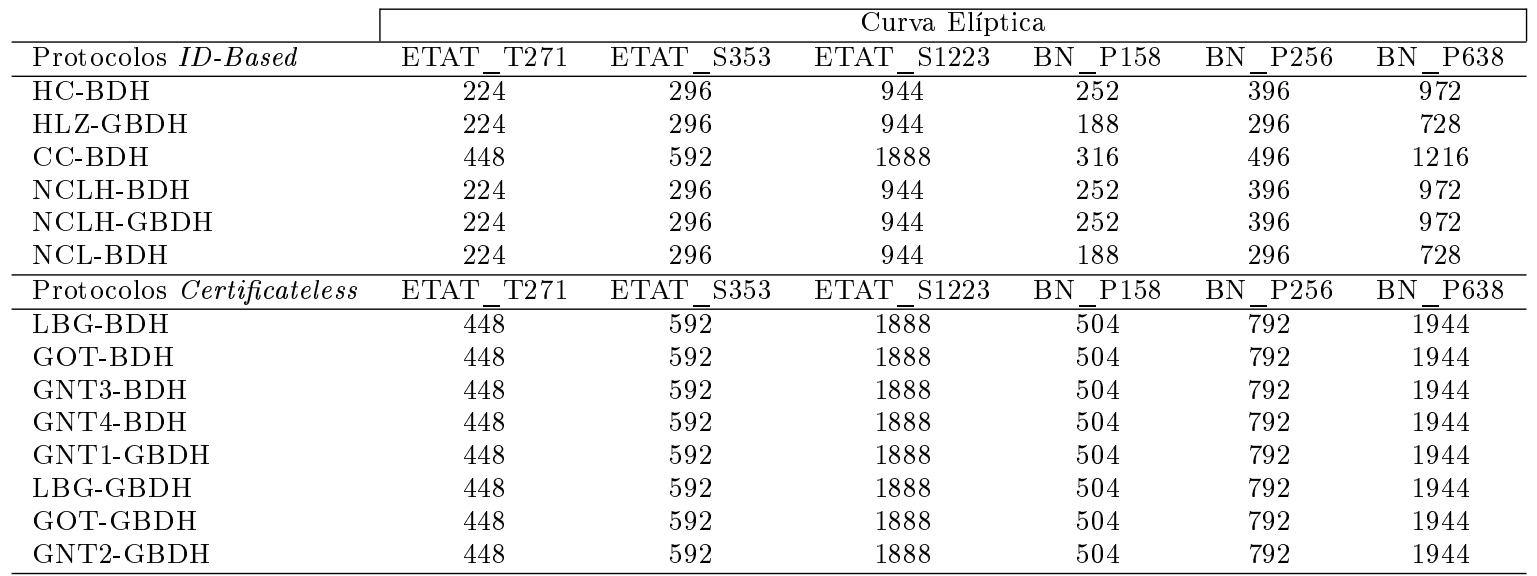

Tabela 6.9: Comprimento das mensagens trocadas (em bytes) dos protocolos analisados para diferentes niveis de segurança.

\subsection{Considerações finais sobre os resultados}

O objetivo dos experimentos relatados ao longo deste capítulo foi comparar o desempenho dos protocolos estudados em diferentes níveis de segurança, e em três dispositivos que dispõem de processadores da arquitetura ARM. Os tempos apresentados na Seção 6.5 representam o tempo médio de execução de uma sessão completa, entre um dispositivo e o servidor, de cada um dos protocolos de acordo de chave selecionados (detalhados no Capítulo 5).

Nos gráficos apresentados pelas Figuras 6.7, 6.8, 6.9 e 6.10, observa-se facilmente que o comportamento dos protocolos é o mesmo entre os diferentes dispositivos, para um mesmo nível de segurança. A diferença de tempo é consequência, principalmente, da diferença de clock de seus processadores. Portanto, não se deve afirmar simplesmente que determinado protocolo é recomendado para um dispositivo, digamos $X$, e não é para outro dispositivo, digamos $Y$. A realização dos experimentos em três dispositivos diferentes foi justamente para mostrar que o comportamento seria mantido entre eles, propiciando maior confiabilidade aos resultados.

A escolha do protocolo deve ser baseada, primeiramente, em qual modelo de criptografia de chave pública será mais recomendado para o cenário proposto. Se não há problema em a autoridade do sistema ter acesso às chaves privadas dos usuários, e se não há perigo dela os personificar, por exemplo, então o modelo baseado em identidade pode ser recomendado. Caso contrário, é desejável utilizar o modelo sem certificado. Feita a escolha do modelo de criptografia, inicia-se a escolha do protocolo propriamente dito.

Recomenda-se sempre escolher o protocolo demonstrado seguro no modelo de segurança mais forte, e sob a hipótese do problema computacional mais difícil. No caso em que houver vários protocolos com estes mesmos parâmetros, a escolha deve-se basear no que apresentou melhor tempo, de acordo com a Tabela 6.6. Se o cenário exige acordos de chave constantes (isto é, várias chaves por minuto, ou mesmo por segundo), recomenda-se pré-computar os valores intermediários que não dependem dos segredos temporários de sessão, e basear-se nos resultados apresentados na Tabela 6.7. Já protocolos em modelos de segurança mais fracos ou sob a hipótese de problema computacional menos difícil podem ser empregados em alguns cenários específicos. Por exemplo, caso seja necessário gerar uma quantidade 
mínima de chaves $n$, em um intervalo de tempo $t$, e que não seja possível alcançar $n$ utilizando protocolos mais seguros, no mesmo dispositivo.

A curva elíptica utilizada (isto é, o nível de segurança empregado) também influenciará diretamente no tempo de execução do protocolo. Conforme a Seção 6.3, curvas primas se mostraram muito mais eficientes do que curvas binárias, e seu uso é encorajado sempre que possível. Além do desempenho, a curva elíptica selecionada afetará na memória e largura de banda utilizadas pelo protocolo, de acordo com os resultados das Tabelas 6.8 e 6.9. Se o dispositivo dispor de pouca memória RAM ou largura de banda, uma curva menor pode ser utilizada. No entanto, a segurança oferecida será mais fraca. Neste caso, as curvas primas também são vantajosas, uma vez que possuem razão nivel de segurança vs memória $R A M$ e nível de segurança vs largura de banda melhores, quando comparadas com curvas binárias. 


\section{Capítulo 7}

\section{Conclusões}

\subsection{Considerações finais}

Este trabalho fez um levantamento de protocolos de acordo de chave com autenticação, nos modelos de criptografia de chave pública baseado em identidade (ID-Based) e sem certificado (Certificateless). Foram apresentadas implementações eficientes dos protocolos estudados, em dispositivos de poder computacional restrito, mostrando que é viável utilizar protocolos relativamente complexos, com níveis elevados de segurança, e tempos de execução praticáveis. Ademais, nossos testes simularam situações do mundo real, incluindo comunicação em uma rede TCP/IP, e uso de bibliotecas criptográficas e matemáticas otimizadas, construídas principalmente nas linguagens C e Assembly. Consequentemente, aplicações construídas em Java, em especial para sistema operacional Android, poderiam utilizar as chaves de sessão geradas, através de chamadas JNI ao código implementado.

Os resultados expostos apontam sugestões para possíveis melhorias de tempo. Dentre elas, sugerimos a substituição de operações de exponenciação no grupo $\mathbb{G}_{T}$ por multiplicações no grupo $\mathbb{G}$. Outra recomendação é a redistribuição das operações de multiplicação nos grupos $\mathbb{G}_{1}$ e $\mathbb{G}_{2}$, quando utilizado emparelhamento assimétrico, de modo que o dispositivo com menor poder computacional efetue o máximo de multiplicações no grupo $\mathbb{G}_{1}$, para ganho de eficiência. Indicamos o uso de curvas elípticas sobre corpos primos sempre que possível, devido ao ganho de eficiência, e melhor utilização de memória e canal de comunicação, em comparação com curvas elípticas sobre corpos binários.

\subsection{Sugestões para pesquisas futuras}

- Apesar da biblioteca criptográfica RELIC-toolkit dispor de suporte a ambientes multicore, as versões do Android NDK disponíveis na época em que os experimentos foram realizados não eram compatíveis com a biblioteca OpenMP (Open Multi-Processing). Internamente, a RELIC-toolkit utiliza OpenMP para paralelização. É possível melhorar os resultados de tempo das operações de emparelhamento em dispositivos com processadores de múltiplos núcleos.

- As versões mais recentes da biblioteca GMP trazem otimizações para processadores da arquitetura ARM. Foram realizadas algumas tentativas para utilizar a versão 5.1.0 desta biblioteca (a mais recente disponível na época em que os experimentos foram realizados), mas não foi possível compilá-la no Android NDK utilizado.

- Os experimentos foram realizados utilizando a versão 0.3.1 da biblioteca RELIC-toolkit, por ser a mais estável para os testes propostos. Versões mais atuais prometem cálculos de emparelhamento mais eficiente. Todavia, tais versões apresentaram alguns erros de compilação ou mesmo erros no resultado das funções. Espera-se que correções e novas melhorias no código da RELIC-toolkit ocorram no futuro, tornando as operações discutidas na Seção 6.3 mais eficientes computacionalmente.

- Experimentos com outros dispositivos que possuam processadores com menor poder computacional, como Arduino, ou dispositivos móveis com processadores da arquitetura Intel Atom. 


\section{Apêndice A}

\section{Fundamentos Matemáticos}

Neste apêndice são apresentados fundamentos matemáticos importantes que são necessários para a compreensão dos conceitos de criptografia de chave pública apresentados ao longo deste trabalho.

\section{A.1 Grupos}

Um grupo $(\mathbb{G}, *)$ é um conjunto não vazio $\mathbb{G}$, com uma operação binária $*$, que combina dois elementos $a, b \in \mathbb{G}$, para obter $a * b \in \mathbb{G}$, satisfazendo as seguintes propriedades (Hoffstein et al., 2008):

1. Existe um elemento identidade (ou elemento neutro) $e \in \mathbb{G}$, tal que: $e * a=a * e=a, \forall a \in \mathbb{G}$.

2. Elemento inverso: $\forall a \in \mathbb{G}$, existe um (único) elemento $\bar{a} \in \mathbb{G}$, chamado inverso de $a$, tal que: $a * \bar{a}=\bar{a} * a=e$.

3. Associatividade: $(a * b) * c=a *(b * c), \forall a, b, c \in \mathbb{G}$.

4. Fechamento: $\forall a, b \in \mathbb{G}, a * b \in \mathbb{G}$.

Se além dessas quatro propriedades a operação $*$ for comutativa (isto é, $a * b=b * a, \forall a, b \in \mathbb{G}$ ), diz-se que $\mathbb{G}$ é um grupo comutativo ou grupo abeliano.

Quando a operação * for substituída pela adição +, o grupo é chamado de grupo aditivo. Sua identidade é representada por 0 , e o inverso de $a$ é representado por $-a$. Define-se multiplicação por escalar de $a \in \mathbb{G}$, por um inteiro $\lambda$, como sendo: $\lambda a=\underbrace{a+a+\cdots+a}_{\lambda \text { vezes }}$.

Quando a operação * for substituída pela multiplicação $\times$, o grupo é chamado de grupo multiplicativo. Sua identidade é representada por 1 , e o inverso de $a$ é representado por $a^{-1}$. Define-se exponenciação de $a \in \mathbb{G}$, por um expoente inteiro $\lambda$, como sendo: $a^{\lambda}=\underbrace{a \times a \times \cdots \times a}_{\lambda \text { vezes }}$

Um grupo aditivo $\mathbb{G}$ é cíclico se existe um elemento $P \in \mathbb{G}$, tal que qualquer elemento $Q \in \mathbb{G}$ pode ser escrito como o produto de $P$ com um escalar inteiro $\lambda$, isto é, $Q=\lambda P$. Um elemento $P$ com essa propriedade é chamado gerador de $\mathbb{G}$.

Se um grupo $\mathbb{G}$ é finito e possui $n$ elementos, tem-se que $|\mathbb{G}|=\# \mathbb{G}=n$ é a ordem de $\mathbb{G}$.

\section{A.2 Anéis}

Um anel é um conjunto não vazio $(\mathbb{A},+, *)$, munido de duas operações binárias: soma + e multiplicação $*$, definidas sobre $\mathbb{G}$, com as seguintes propriedades (Terada, 2008):

1. $(\mathbb{A},+)$ é um grupo aditivo comutativo com identidade 0 .

2. A operação $*$ é associativa: $(a * b) * c=a *(b * c), \forall a, b, c \in \mathbb{A}$.

3. Existe um elemento identidade $1 \in \mathbb{A}, 1 \neq 0$, tal que: $a * 1=1 * a=a, \forall a \in \mathbb{A}$.

4. A operação $*$ é distributiva sobre $+: a *(b+c)=(a * b)+(a * c)$, e $(b+c) * a=(b * a)+(c * a), \forall a, b, c \in \mathbb{A}$.

5. $\mathbb{A}$ é comutativo se $\forall a, b \in \mathbb{G}: a * b=b * a$.

Um elemento $a \in \mathbb{A}$ é chamado unidade (ou elemento inversível) se existe $b \in \mathbb{A}$, tal que $a * b=1$. O conjunto das unidades de $\mathbb{A}$ forma um grupo chamado grupo das unidades de $\mathbb{A}$. Um exemplo é o grupo das unidades módulo $m:(\mathbb{Z} / m \mathbb{Z})^{*}=\{a \in \mathbb{Z} / m \mathbb{Z}: m d c(a, m)=1\}$ (Hoffstein et al., 2008). 


\section{A.3 Corpos}

Um corpo $\mathcal{K}$ é um anel comutativo no qual todos os elementos não nulos possuem inverso multiplicativo, i.e.: $\forall a \in \mathcal{K}, a \neq 0 \Rightarrow \exists b \in \mathcal{K}: a b=1$.

As seguintes propriedades são válidas:

1. $(\mathcal{K},+)$ é um grupo abeliano com identidade (aditiva) 0 .

2. $(\mathcal{K} \backslash\{0\}, *)$ é um grupo abeliano com identidade (multiplicativa) 1 .

3. Distributividade: $(a+b) * c=a * c+b * c, \forall a, b, c \in \mathcal{K}$.

4. As identidades aditiva e multiplicativa são distintas, i.e.: $0 \neq 1$.

A característica de um corpo, denotada por $\operatorname{char}(\mathcal{K})$, é o menor número de vezes que a identidade multiplicativa de $\mathcal{K}$ deve ser somada a si mesma para que o resultado seja igual à sua identidade aditiva. Ou seja, $\operatorname{char}(\mathcal{K})$ é o menor $n$ positivo tal que:

$$
\underbrace{1+1+\cdots+1}_{n \text { vezes }}=0
$$

Caso o resultado dessa soma nunca seja a identidade aditiva ,define-se $\operatorname{char}(\mathcal{K})=0$.

\section{A.3.1 Corpos finitos}

Quando o número de elementos de um corpo é finito, ele é denominado corpo finito ou corpo finito de Galois. ${ }^{1}$ Tal número de elementos é denominado ordem do corpo.

Algumas propriedades importantes dos corpos finitos são:

1. A ordem $q$ de um corpo finito é sempre da forma $p^{m}$, onde $p$ é um número primo, chamado de característica do corpo, e $m$ é um inteiro positivo.

2. Para cada número primo $p$ e cada inteiro positivo $m$, existe um único corpo finito (a menos de isomorfismo) com $p^{m}$ elementos.

3. Quaisquer dois corpos finitos que possuam mesma ordem são isomorfos.

De maneira resumida, dizemos que existe isomorfismo entre dois corpos finitos quando há uma função bijetora que mapeia elementos identidade e inversos entre eles, conservando suas propriedades ou operações.

Corpos finitos são geralmente representados na literatura por $\mathbb{F}_{q}$ ou $G F\left(p^{m}\right) .^{2}$ Para o caso em que o número de elementos é primo, i.e., $m=1$, escreve-se $\mathbb{F}_{p}$ ou $G F(p)$. Tal corpo é denominado corpo primo.

Outro caso especial são os corpos da forma $\mathbb{F}_{2^{m}}$, i.e., $p=2$, denominados de corpos binários ou corpos de característica 2. Tais corpos são utilizados com frequência em aplicações de criptografia. Uma maneira de construí-los é utilizando a representação de base polinomial:

$$
a_{m-1} x^{m-1}+a_{m-2} x^{m-2}+\cdots+a_{2} x^{2}+a_{1} x^{1}+a_{0}
$$

onde $a_{i} \in \mathbb{F}_{2}=\{0,1\}$.

Devido à natureza binária dos computadores, os corpos binários permitem a utilização direta de bits em memória para representar os coeficientes de um elemento em $\mathbb{F}_{2^{m}}$, sem necessidade de definir estruturas de dados mais complexas (Hoffstein et al., 2008).

\section{A.4 Curvas elípticas}

Uma curva elíptica $E$ sobre um corpo $\mathcal{K}$, representada por $E(\mathcal{K})$ ou $E / \mathcal{K}$, é definida pela equação (Hankerson et al. , 2003):

$$
E: y^{2}+a_{1} x y+a_{3} y=x^{3}+a_{2} x^{2}+a_{4} x+a_{6}
$$

onde $a_{1}, a_{2}, a_{3}, a_{4}, a_{6} \in \mathcal{K}$ e $\Delta \neq 0$, onde $\Delta$ é o discriminante de $E$, definido como:

$$
\left.\begin{array}{rl}
\Delta & =-d_{2}^{2} d_{8}-8 d_{4}^{3}-27 d_{6}^{2}+9 d_{2} d_{4} d_{6} \\
d_{2} & =a_{1}^{2}+4 a_{2} \\
d_{4} & =2 a_{4}+a_{1} a_{3} \\
d_{6} & =a_{3}^{2}+4 a_{6} \\
d_{8} & =a_{1}^{2} a_{6}+4 a_{2} a_{6}-a_{1} a_{3} a_{4}+a_{2} a_{3}^{2}-a_{4}^{2}
\end{array}\right\}
$$

\footnotetext{
${ }^{1}$ Em homenagem ao matemático francês Évariste Galois (1811-1832).

${ }^{2}$ Do inglês Galois Field.
} 
A curva elíptica $E(\mathcal{K})$ é o conjunto dos pares $(x, y) \in \mathcal{K} \times \mathcal{K}$ que satisfazem a equação A.2, mais um ponto $\mathcal{O}$, chamado de ponto no infinito. O discriminante $\Delta \neq 0$ garante que não existirão duas ou mais retas tangentes distintas para um ponto na curva.

A equação A.2 é chamada de equação de Weierstrass ${ }^{3}$, e pode ser simplificada a depender da característica do corpo finito utilizado.

Se $\operatorname{char}(\mathcal{K})=2$, i.e., $\mathcal{K}=G F\left(2^{m}\right)$, a equação A.2 pode ser reduzida, para dois casos a serem considerados:

$$
E: \begin{cases}y^{2}+x y=x^{3}+a x^{2}+b, & \text { se } a_{1} \neq 0 \\ y^{2}+c y=x^{3}+a x+b, & \text { se } a_{1}=0\end{cases}
$$

onde $a, b, c \in G F\left(2^{m}\right)$. Para o caso em que $a_{1} \neq 0$, curva é dita não-supersingular e possui discriminante $\Delta=b$. Já quando $a_{1}=0$, é dita supersingular, com discriminante $\Delta=c^{4}$.

Se $\operatorname{char}(\mathcal{K})=3$, i.e., $\mathcal{K}=G F\left(3^{m}\right)$, também existem dois casos a serem considerados. A equação A.2 pode ser reescrita como:

$$
E: \begin{cases}y^{2}=x^{3}+a x^{2}+b, & \text { se } a_{1}^{2} \neq-a_{2} \\ y^{2}=x^{3}+a x+b, & \text { se } a_{1}^{2}=-a_{2}\end{cases}
$$

onde $a, b \in G F\left(3^{m}\right)$. Quando $a_{1}^{2} \neq-a_{2}$, a curva é dita não-supersingular e possui discriminante $\Delta=-a^{3} b$. Se $a_{1}^{2}=-a_{2}$, a curva é dita supersingular e possui discriminante $\Delta=-a^{3}$.

Se $\operatorname{char}(\mathcal{K})>3$, para um inteiro $p$ primo, $p>3$, i.e.GF $\left(p^{m}\right)$, a equação A.2 é reduzida para:

$$
E: y^{2}=x^{3}+a x+b
$$

onde $a, b \in G F\left(p^{m}\right)$, com discriminante $\Delta=-16\left(4 a^{3}+27 b^{2}\right)$.

O uso de curvas elípticas em criptografia foi proposto independentemente por Victor S. Miller (Miller, 1986) e Neal Koblitz (Koblitz, 1987).

\section{A.4.1 Ordem de curvas elípticas}

Seja $E$ uma curva elíptica definida sobre um corpo finito $\mathbb{F}_{q}$. O número de pontos em $E\left(\mathbb{F}_{q}\right)$, representado por $\# E\left(\mathbb{F}_{q}\right)$, é chamado ordem de $E$ sobre $\mathbb{F}_{q}$.

Pela equação de Weierstrass, em A.2, cada $x \in \mathbb{F}_{q}$ possui no máximo duas soluções, então: \# $E\left(\mathbb{F}_{q}\right) \in[1,2 q+1]$. De acordo com o Teorema de Hasse é possível calcular um intervalo mais preciso para determinar $\# E\left(\mathbb{F}_{q}\right)$ : (Hankerson et al., 2003)

$$
q+1-2 \sqrt{q} \leq \# E\left(\mathbb{F}_{q}\right) \leq q+1+2 \sqrt{q}
$$

Uma outra forma de escrever o Teorema de Hasse é:

$$
\# E\left(\mathbb{F}_{q}\right)=q+1-t
$$

onde $|t| \leq 2 \sqrt{q}$ é denominado traço de Frobenius. Como o resultado de $2 \sqrt{q}$ é relativamente pequeno quando comparado com $q$, tem-se que $\# E\left(\mathbb{F}_{q}\right) \approx q$.

A ordem de uma curva elíptica $E\left(\mathbb{F}_{q}\right)$ fornece informações importantes sobre algumas das propriedades da curva utilizada. Quando $\# E\left(\mathbb{F}_{q}\right)=q$, diz-se que $E$ é anômala. Este tipo de curva não é apropriada para uso em sistemas criptográficos. Outra propriedade possível de se determinar é a supersingularidade de $E$.

Seja $p$ a característica de $\mathbb{F}_{q}$, e seja a curva elíptica $E\left(\mathbb{F}_{q}\right)$. Uma curva elíptica é dita supersingular se seu traço de Frobenius $t$ é múltiplo de $p$, ou seja, se $p \mid t$. O uso desse tipo de curva em sistemas criptográficos deve ser evitado, pois oferece nível de segurança inferior quando comparado a outros tipos de curvas elípticas, para chaves criptográficas de mesmo comprimento.

\section{A.4.2 Lei de grupos em curvas elípticas}

Seja $E$ uma curva elíptica definida sobre um corpo finito $\mathbb{F}_{q}$, representada por $E\left(\mathbb{F}_{q}\right)$, e sejam $P, Q \in E\left(\mathbb{F}_{q}\right)$, pontos da curva $E$. É possível somar dois pontos $P$ e $Q$ e obter um terceiro ponto, $R \in E\left(\mathbb{F}_{q}\right)$. O conjunto de pontos de $E\left(\mathbb{F}_{q}\right)$, a operação de soma, e um ponto especial $\mathcal{O}$, que representa a identidade de $E\left(\mathbb{F}_{q}\right)$, formam um grupo abeliano.

As regras de adição entre dois pontos $P=\left(x_{1}, y_{1}\right)$ e $Q=\left(x_{2}, y_{2}\right)$ são definidas algebricamente da seguinte forma:

1. $P+\mathcal{O}=\mathcal{O}+P=P$, pois $\mathcal{O}$ é o elemento neutro.

\footnotetext{
${ }^{3}$ Em homenagem ao matemático alemão Karl Weierstrass (1815-1897)
} 
2. Se $x_{1}=x_{2}$ e $-y_{1}=y_{2}$ (ou seja, $P=-Q$ ), então $P+Q=\mathcal{O}$.

3. Se $P \neq-Q$, então: $P+Q=\left(x_{3}, y_{3}\right)$, onde $\left\{\begin{array}{l}\begin{array}{l}x_{3}=\lambda^{2}-x_{1}-x_{2} \\ y_{3}=\lambda\left(x_{1}-x_{3}\right)-y_{1}\end{array} \\ \lambda=\left\{\begin{array}{l}\frac{y_{2}-y_{1}}{x_{2}-x_{1}}, \quad \text { se } P \neq Q \\ \frac{3\left(x_{1}\right)^{2}+a}{2 y_{1}}, \text { se } P=Q\end{array}\right.\end{array}\right.$

Observa-se que o inverso de um ponto $P=(x, y)$ é denotado por $-P=(x,-y)=-(x, y)$.

Com a operação de adição definida, os pontos da curva formarão um grupo comutativo, com $\mathcal{O}$ como elemento identidade.

Geometricamente, para somar os pontos $P$ e $Q$, tal que $P \neq \pm Q$ e $P, Q \neq \mathcal{O}$, traça-se uma reta através dos pontos $P$ e $Q$, interceptando um terceiro ponto da curva elíptica $E\left(\mathbb{F}_{q}\right)$, que chamaremos de $-R=\left(x_{3},-y_{3}\right)$. Logo em seguida, calcula-se o ponto simétrico de $-R$ em relação ao eixo das abscissas, ou seja, $R=\left(x_{3}, y_{3}\right)$. O ponto $R$ é a soma de $P$ e $Q$.

Já para dobrar um ponto $P$ (caso em que $P=Q$ ), traça-se uma linha tangente ao ponto $P$, que intercepta um segundo ponto $-R \in E\left(\mathbb{F}_{q}\right)$. De forma análoga à adição, calcula-se o ponto simétrico de $-R$ em relação ao eixo das abscissas, isto é, $R$. A Figura A.1 ilustra as operações de soma e dobramento em $E\left(\mathbb{F}_{q}\right)$.
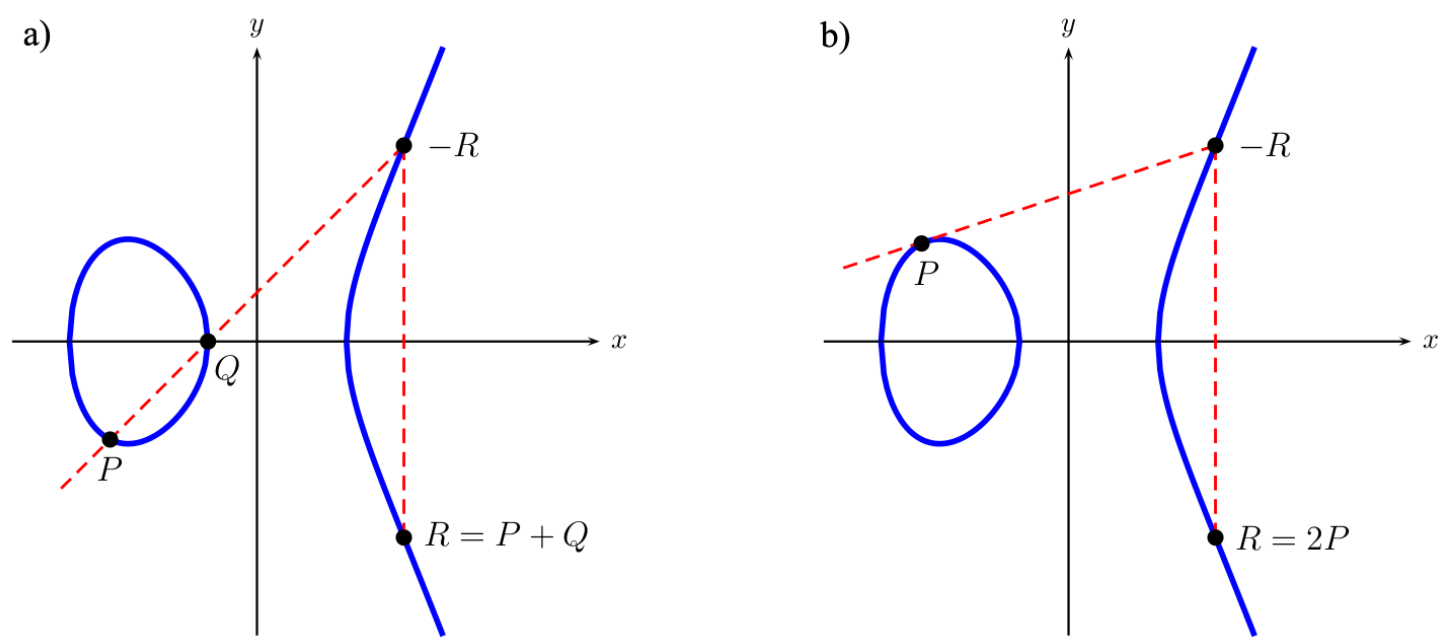

Figura A.1: a) soma entre dois pontos $P$ e $Q$ e b) dobramento de um ponto $P$ 


\section{Apêndice B}

\section{Intervalos de Confiança dos Experimentos}

As Tabelas B.1, B.2 e B.3 apresentam os intervalos de confiança dos experimentos apresentados no Capítulo 6. As colunas da Tabela B.3 estão de acordo com as descrições da Seção 6.3.

A metodologia utilizada para o cálculo dos intervalos de confiança é descrita de acordo com a equação a seguir:

$$
I C=\left[\bar{x}_{n}-t_{n-1} \frac{S_{n}}{\sqrt{n}}, \bar{x}_{n}+t_{n-1} \frac{S_{n}}{\sqrt{n}}\right]
$$

Onde:

- $n$ é a quantidade de amostras.

- $\bar{x}_{n}$ é a média de $n$ amostras.

- $t_{n-1}$ é o valor do 97.5 percentil da distribuição $t$ de Student, com $n-1$ graus de liberdade.

- $S_{n}$ é o desvio padrão das $n$ amostras.

- $I C$ é o intervalo de confiança.

Nos experimentos realizados o valor de $n$ é sempre igual a 50 (conforme descrito nas seções 6.3 e 6.5). Portanto o valor de $t_{n-1}$ é, aproximadamente, 2.009575 . 


\begin{tabular}{|c|c|c|c|c|c|c|c|}
\hline \multirow{3}{*}{$\begin{array}{l}\text { Protocolos ID-Based } \\
\text { HC-BDH }\end{array}$} & \multirow[b]{2}{*}{ Dispositivo } & \multicolumn{6}{|c|}{ Curva Elíptica } \\
\hline & & ETAT_T271 & ETAT_S353 & ETAT_S1223 & $\mathrm{BN} \_\mathrm{P} 158$ & BN_P256 & $\mathrm{BN}$ P638 \\
\hline & $\mathrm{RPi}$ & {$[181.7,182.9]$} & {$[323.8, \overline{3} 25.4]$} & {$[4813.0,4829.9]$} & {$[103 . \overline{6}, 104.6]$} & {$[218 . \overline{4}, 218.9]$} & {$[1430 . \overline{9}, 1431.9]$} \\
\hline & MM1 & {$[178.8,180.2]$} & {$[326.6,333.1]$} & {$[4503.9,4526.1]$} & {$[111.8,113.0]$} & {$[227.4,229.0]$} & {$[1422.1,1426.9]$} \\
\hline & GN7 & {$[71.2,72.9]$} & {$[121.9,124.1]$} & {$[1871.6,1900.0]$} & {$[48.0,50.0]$} & {$[93.0,94.9]$} & {$[526.7,531.2]$} \\
\hline \multirow[t]{3}{*}{ HLZ-GBDH } & $\mathrm{RPi}$ & {$[150.8,151.5]$} & {$[247.7,248.9]$} & {$[3004.7,3016.6]$} & {$[94.7,94.9]$} & {$[206.2,206.6]$} & {$[1318.8,1319.8]$} \\
\hline & MM1 & {$[146.7,148.0]$} & {$[242.3,244.8]$} & {$[2817.7,2850.4]$} & {$[104.2,107.0]$} & {$[213.9,216.0]$} & {$[1339.1,1372.7]$} \\
\hline & GN 7 & {$[58.4,59.7]$} & {$[94.2,96.7]$} & {$[1184.5,1192.0]$} & {$[44.6,46.9]$} & {$[86.5,88.7]$} & {$[482.5,485.6]$} \\
\hline \multirow[t]{3}{*}{$\mathrm{CC}-\mathrm{BDH}$} & $\mathrm{RPi}$ & {$[198.5,200.0]$} & {$[340.9,343.0]$} & {$[4528.2,4550.1]$} & {$[80.7,81.1]$} & {$[175.1,175.8]$} & {$[1013.7,1016.0]$} \\
\hline & MM1 & {$[198.4,202.8]$} & {$[337.7,347.7]$} & {$[4329.5,4374.8]$} & {$[88.5,91.4]$} & {$[184.0,186.1]$} & {$[1060.9,1131.9]$} \\
\hline & GN7 & {$[78.7,79.5]$} & {$[130.7,133.6]$} & {$[1761.0,1769.9]$} & {$[36.4,38.8]$} & {$[74.9,76.1]$} & {$[381.2,382.9]$} \\
\hline \multirow[t]{2}{*}{ NCLH-BDH } & $\mathrm{RPi}$ & {$[440.8,444.0]$} & {$[779.8,784.7]$} & {$[12112.2,12157.0]$} & {$[344.4,346.6]$} & {$[780.9,784.1]$} & {$[5037.5,5051.7]$} \\
\hline & GN7 & {$[163.3,165.8]$} & {$[286.5,289.8]$} & {$[4741.6,4765.3]$} & {$[144.3,146.5]$} & {$[319.4,321.7]$} & {$[1819.0,1825.2]$} \\
\hline \multirow[t]{3}{*}{$\mathrm{NCLH}-\mathrm{GBDH}$} & $\mathrm{RPi}$ & {$[249.0,250.8]$} & {$[448.9,451.6]$} & {$[6813.4,6839.0]$} & {$[177.1,178.3]$} & {$[402.4,404.1]$} & {$[2583.2,2590.8]$} \\
\hline & MM1 & {$[240.3,247.2]$} & {$[427.7,431.9]$} & {$[6344.7,6373.2]$} & {$[194.7,198.7]$} & {$[403.8,406.0]$} & {$[2552.5,2563.9]$} \\
\hline & GN7 & {$[94.5,95.4]$} & {$[158.4,161.0]$} & {$[2673.0,2688.3]$} & {$[75.2,77.3]$} & {$[161.5,165.3]$} & {$[934.1,940.3]$} \\
\hline \multirow{3}{*}{ NCL-BDH } & $\mathrm{RPi}$ & {$[151.2,152.0]$} & {$[270.6,271.6]$} & {$[4034.6,4044.6]$} & {$[94.8,95.0]$} & {$[217.2,217.6]$} & {$[1364.3,1364.9]$} \\
\hline & MM1 & {$[147.4,151.7]$} & {$[267.2,274.2]$} & {$[3764.6,3785.9]$} & {$[105.2,107.8]$} & {$[214.8,216.3]$} & {$[1346.0,1352.4]$} \\
\hline & GN7 & {$[59.8,61.6]$} & {$[103.5,105.4]$} & {$[1571.8,1595.0]$} & {$[44.8,48.2]$} & {$[86.0,86.9]$} & {$[496.4,499.6]$} \\
\hline Protocolos Certificateless & Dispositivo & ETAT_T271 & ETAT_S353 & ETAT_S1223 & $\mathrm{BN} \_\mathrm{P} 158$ & $\mathrm{BN} \_\mathrm{P} 256$ & $\mathrm{BN} \_\mathrm{P} 638$ \\
\hline \multirow[t]{2}{*}{ LBG-BDH } & $\mathrm{RPi}$ & {$[677.2,679.7]$} & {$[1185.4,1188.6]$} & {$[17701.9,17734.2]$} & {$[464 . \overline{2}, 465.7]$} & {$[1034 . \overline{0}, 1034.9]$} & {$[6815 . \overline{3}, 6818.9]$} \\
\hline & MM1 & {$[635.7,638.8]$} & {$[1123.6,1134.4]$} & {$[16601.0,16776.0]$} & {$[489.0,501.6]$} & {$[1065.0,1072.7]$} & {$[6558.5,6573.1]$} \\
\hline \multirow{2}{*}{ GOT-BDH } & GN7 & {$[212.4,215.3]$} & {$[374.0,377.0]$} & {$[5967.9,5981.8]$} & {$[162.9,164.4]$} & {$[347.3,349.9]$} & {$[1972.8,1978.2]$} \\
\hline & GN7 & {$[254.3,259.7]$} & {$[427.4,431.2]$} & {$[6925.2,6937.5]$} & {$[198.9,200.0]$} & {$[421.6,424.2]$} & {$[2419.3,2424.3]$} \\
\hline \multirow[t]{3}{*}{ GNT3-BDH } & $\mathrm{RPi}$ & {$[493.3,495.5]$} & {$[869.8,873.0]$} & {$[12863.2,12895.7]$} & {$[284.3,284.7]$} & {$[640.2,640.9]$} & {$[4306.0,4309.2]$} \\
\hline & MM1 & {$[469.8,475.7]$} & {$[824.1,829.3]$} & {$[11951.1,12012.6]$} & {$[310.9,316.4]$} & {$[673.7,676.8]$} & {$[4131.5,4145.2]$} \\
\hline & GN7 & {$[182.9,183.8]$} & {$[318.9,321.5]$} & {$[5000.4,5023.2]$} & {$[126.6,128.6]$} & {$[270.2,272.9]$} & {$[1523.4,1528.7]$} \\
\hline \multirow[t]{3}{*}{ GNT4-BDH } & $\mathrm{RPi}$ & {$[401.1,403.4]$} & {$[711.0,714.2]$} & {$[10383.7,10416.6]$} & {$[209.5,210.0]$} & {$[460.1,460.7]$} & {$[3009.3,3012.1]$} \\
\hline & MM1 & {$[384.6,387.8]$} & {$[685.1,689.4]$} & {$[9690.8,9729.4]$} & {$[224.1,230.2]$} & {$[477.0,483.5]$} & {$[2918.2,2924.3]$} \\
\hline & GN7 & {$[150.5,151.7]$} & {$[265.9,268.2]$} & {$[4042.5,4057.4]$} & {$[93.1,95.4]$} & {$[186.5,189.2]$} & {$[1080.8,1086.9]$} \\
\hline \multirow{3}{*}{ GNT1-GBDH } & $\mathrm{RPi}$ & {$[409.0,411.6]$} & {$[710.5,713.9]$} & {$[10365.8,10397.9]$} & {$[215.2,215.6]$} & {$[451.2,451.8]$} & {$[3009.1,3012.6]$} \\
\hline & MM1 & {$[383.1,386.2]$} & {$[680.1,695.4]$} & {$[9673.9,9721.3]$} & {$[222.6,224.9]$} & {$[475.7,480.3]$} & {$[2912.8,2928.3]$} \\
\hline & GN7 & {$[149.7,151.0]$} & {$[264.5,266.6]$} & {$[4045.5,4062.6]$} & {$[91.9,93.4]$} & {$[187.9,189.1]$} & {$[1079.6,1085.8]$} \\
\hline \multirow[t]{3}{*}{ LBG-GBDH } & $\mathrm{RPi}$ & {$[445.8,448.1]$} & {$[789.6,792.7]$} & {$[11575.7,11608.9]$} & {$[243.1,243.6]$} & {$[563.0,563.5]$} & {$[3634.6,3637.7]$} \\
\hline & MM1 & {$[423.5,428.5]$} & {$[747.6,755.7]$} & {$[10809.1,10857.9]$} & {$[265.5,268.9]$} & {$[577.1,584.0]$} & {$[3522.9,3539.3]$} \\
\hline & GN7 & {$[165.3,166.7]$} & {$[288.6,292.3]$} & {$[4518.4,4530.7]$} & {$[110.1,113.8]$} & {$[230.4,233.7]$} & {$[1299.8,1303.1]$} \\
\hline \multirow[t]{3}{*}{ GOT-GBDH } & $\mathrm{RPi}$ & {$[398.5,400.8]$} & {$[709.4,712.6]$} & {$[10350.1,10383.3]$} & {$[207.6,208.0]$} & {$[468.6,469.1]$} & {$[2975.1,2977.4]$} \\
\hline & MM1 & {$[381.8,385.7]$} & {$[674.5,680.0]$} & {$[9679.9,9734.8]$} & {$[222.3,227.4]$} & {$[475.5,482.8]$} & {$[2913.6,2938.1]$} \\
\hline & GN7 & {$[149.0,150.9]$} & {$[264.2,267.2]$} & {$[4037.4,4050.5]$} & {$[92.4,95.4]$} & {$[187.3,188.3]$} & {$[1080.7,1084.4]$} \\
\hline \multirow{3}{*}{ GNT2-GBDH } & $\mathrm{RPi}$ & {$[353.0,355.2]$} & {$[632.3,635.5]$} & {$[9145.9,9180.7]$} & {$[160.7,161.1]$} & {$[357.0,357.6]$} & {$[2366.7,2368.8]$} \\
\hline & MM1 & {$[342.5,349.3]$} & {$[603.2,607.8]$} & {$[8539.7,8589.3]$} & {$[179.7,189.4]$} & {$[378.2,381.2]$} & {$[2307.3,2315.1]$} \\
\hline & GN7 & {$[133.2,134.6]$} & {$[229.6,231.8]$} & {$[3559.6,3571.2]$} & {$[76.8,78.9]$} & {$[149.5,151.1]$} & {$[858.4,862.9]$} \\
\hline
\end{tabular}

Tabela B.1: Intervalo de confiança (95\%) dos tempos de execução dos protocolos (em milissegundos), sem pré-computação, para diferentes níveis de segurança. 


\begin{tabular}{|c|c|c|c|c|c|c|c|}
\hline \multirow[b]{2}{*}{ Protocolos ID-Based } & \multirow[b]{2}{*}{ Dispositivo } & \multicolumn{6}{|c|}{ Curva Elíptica } \\
\hline & & ETAT_T271 & ETAT_S353 & ETAT_S1223 & BN_P158 & BN_P256 & BN_P638 \\
\hline \multirow[t]{3}{*}{$\mathrm{HC}-\mathrm{BDH}$} & $\mathrm{RPi}$ & {$[181.7,182.9]$} & {$[323.8,325.4]$} & {$[4813.0,4829.9]$} & {$[103 . \overline{6}, 104.6]$} & {$[218 . \overline{4}, 218.9]$} & {$[1430 . \overline{9}, 1431.9]$} \\
\hline & MM1 & {$[178.8,180.2]$} & {$[326.6,333.1]$} & {$[4503.9,4526.1]$} & {$[111.8,113.0]$} & {$[227.4,229.0]$} & {$[1422.1,1426.9]$} \\
\hline & GN7 & {$[71.2,72.9]$} & {$[121.9,124.1]$} & {$[1871.6,1900.0]$} & {$[48.0,50.0]$} & {$[93.0,94.9]$} & {$[526.7,531.2]$} \\
\hline \multirow[t]{3}{*}{ HLZ-GBDH } & $\mathrm{RPi}$ & {$[150.8,151.5]$} & {$[247.7,248.9]$} & {$[3004.7,3016.6]$} & {$[94.7,94.9]$} & {$[206.2,206.6]$} & {$[1318.8,1319.8]$} \\
\hline & MM1 & {$[146.7,148.0]$} & {$[242.3,244.8]$} & {$[2817.7,2850.4]$} & {$[104.2,107.0]$} & {$[213.9,216.0]$} & {$[1339.1,1372.7]$} \\
\hline & GN7 7 & {$[58.4,59.7]$} & {$[94.2,96.7]$} & {$[1184.5,1192.0]$} & {$[44.6,46.9]$} & {$[86.5,88.7]$} & {$[482.5,485.6]$} \\
\hline \multirow[t]{3}{*}{$\mathrm{CC}-\mathrm{BDH}$} & $\mathrm{RPi}$ & {$[198.5,200.0]$} & {$[340.9,343.0]$} & {$[4528.2,4550.1]$} & {$[80.7,81.1]$} & {$[175.1,175.8]$} & {$[1013.7,1016.0]$} \\
\hline & MM1 & {$[198.4,202.8]$} & {$[337.7,347.7]$} & {$[4329.5,4374.8]$} & {$[88.5,91.4]$} & {$[184.0,186.1]$} & {$[1060.9,1131.9]$} \\
\hline & GN 7 & {$[78.7,79.5]$} & {$[130.7,133.6]$} & {$[1761.0,1769.9]$} & {$[36.4,38.8]$} & {$[74.9,76.1]$} & {$[381.2,382.9]$} \\
\hline \multirow[t]{3}{*}{$\mathrm{NCLH}-\mathrm{BDH}$} & $\mathrm{RPi}$ & {$[256.8,260.6]$} & {$[464.5,469.8]$} & {$[7160.1,7211.7]$} & {$[174.4,176.4]$} & {$[396.8,400.2]$} & {$[2568.9,2578.9]$} \\
\hline & MM1 & {$[247.3,250.9]$} & {$[442.3,449.1]$} & {$[7079.5,7341.8]$} & {$[194.6,197.3]$} & {$[400.2,405.8]$} & {$[2556.8,2588.5]$} \\
\hline & GN7 & {$[97.6,99.1]$} & {$[168.0,171.7]$} & {$[2817.2,2838.6]$} & {$[76.9,78.0]$} & {$[162.1,163.7]$} & {$[940.3,947.2]$} \\
\hline \multirow{3}{*}{ NCLH-GBDH } & $\mathrm{RPi}$ & {$[157.0,159.1]$} & {$[289.6,292.6]$} & {$[4341.7,4370.0]$} & {$[92.3,93.3]$} & {$[209.1,210.8]$} & {$[1343.7,1348.9]$} \\
\hline & MM1 & {$[153.8,156.3]$} & {$[274.6,278.4]$} & {$[4089.9,4128.2]$} & {$[105.1,106.6]$} & {$[212.1,217.6]$} & {$[1432.7,1455.7]$} \\
\hline & GN7 & {$[62.1,63.4]$} & {$[104.9,106.1]$} & {$[1710.1,1723.7]$} & {$[44.5,46.1]$} & {$[88.0,89.3]$} & {$[501.5,506.2]$} \\
\hline \multirow[t]{3}{*}{ NCL-BDH } & $\mathrm{RPi}$ & {$[151.2,152.0]$} & {$[270.6,271.6]$} & {$[4034.6,4044.6]$} & {$[94.8,95.0]$} & {$[217.2,217.6]$} & {$[1364.3,1364.9]$} \\
\hline & MM1 & {$[147.4,151.7]$} & {$[267.2,274.2]$} & {$[3764.6,3785.9]$} & {$[105.2,107.8]$} & {$[214.8,216.3]$} & {$[1346.0,1352.4]$} \\
\hline & GN7 & {$[59.8,61.6]$} & {$[103.5,105.4]$} & {$[1571.8,1595.0]$} & {$[44.8,48.2]$} & {$[86.0,86.9]$} & {$[496.4,499.6]$} \\
\hline Protocolos Certificateless & Dispositivo & ETAT_T271 & ETAT_S353 & ETAT_S1223 & BN_P158 & BN_P256 & $\mathrm{BN} \_\mathrm{P} 638$ \\
\hline \multirow{3}{*}{ LBG-BDH } & $\mathrm{RPi}$ & {$[338.6,340.3]$} & {$[597.5,-600.0]$} & {$[8784.0,-8805.4]$} & {$[197 . \overline{1}, 197.5]$} & {$[437 . \overline{2}, 438.0]$} & {$[2870 . \overline{3}, 2871.9]$} \\
\hline & MM1 & {$[321.8,326.7]$} & {$[567.4,574.0]$} & {$[8218.3,8301.6]$} & {$[211.1,231.9]$} & {$[456.6,460.8]$} & {$[2779.9,2802.3]$} \\
\hline & GN7 & {$[126.1,127.0]$} & {$[214.0,218.8]$} & {$[3430.0,3445.0]$} & {$[88.7,89.5]$} & {$[179.2,180.1]$} & {$[1029.3,1032.5]$} \\
\hline \multirow[t]{3}{*}{ GOT-BDH } & $\mathrm{RPi}$ & {$[245.6,247.3]$} & {$[442.0,444.3]$} & {$[6353.5,6375.0]$} & {$[115.5,115.8]$} & {$[244.1,244.6]$} & {$[1629.7,1631.9]$} \\
\hline & MM1 & {$[238.4,242.8]$} & {$[423.5,429.0]$} & {$[5944.8,6001.3]$} & {$[126.4,128.3]$} & {$[267.2,285.2]$} & {$[1559.7,1565.4]$} \\
\hline & GN7 7 & {$[94.2,95.4]$} & {$[159.7,160.6]$} & {$[2484.5,2495.1]$} & {$[53.8,55.1]$} & {$[104.3,105.5]$} & {$[583.4,586.3]$} \\
\hline \multirow[t]{3}{*}{ GNT3-BDH } & $\mathrm{RPi}$ & {$[247.4,249.1]$} & {$[440.4,442.8]$} & {$[6373.7,6394.7]$} & {$[108.2,108.5]$} & {$[242.2,242.8]$} & {$[1612.5,1614.4]$} \\
\hline & MM1 & {$[238.7,243.2]$} & {$[421.1,425.8]$} & {$[5937.6,5983.0]$} & {$[124.4,126.7]$} & {$[261.2,265.4]$} & {$[1564.5,1573.7]$} \\
\hline & GN7 & {$[94.1,95.1]$} & {$[159.7,160.6]$} & {$[2435.0,2812.5]$} & {$[53.8,55.2]$} & {$[103.8,104.6]$} & {$[585.7,588.7]$} \\
\hline \multirow[t]{3}{*}{ GNT4-BDH } & $\mathrm{RPi}$ & {$[246.2,248.3]$} & {$[439.2,441.7]$} & {$[6350.6,6372.1]$} & {$[112.3,112.7]$} & {$[245.4,245.9]$} & {$[1600.8,1602.4]$} \\
\hline & MM1 & {$[238.7,241.1]$} & {$[422.9,427.7]$} & {$[5935.7,5965.8]$} & {$[127.6,130.2]$} & {$[260.1,263.4]$} & {$[1561.9,1569.7]$} \\
\hline & GN 7 & {$[94.2,95.0]$} & {$[160.2,161.2]$} & {$[2480.8,2492.9]$} & {$[56.1,58.5]$} & {$[103.7,104.9]$} & {$[586.9,589.2]$} \\
\hline \multirow[t]{3}{*}{ GNT1-GBDH } & $\mathrm{RPi}$ & {$[252.4,254.1]$} & {$[440.5,442.9]$} & {$[6350.0,6371.9]$} & {$[115.6,116.1]$} & {$[241.7,242.2]$} & {$[1602.1,1603.8]$} \\
\hline & MM1 & {$[240.6,247.9]$} & {$[419.6,422.9]$} & {$[5932.0,5957.0]$} & {$[127.0,129.5]$} & {$[260.1,262.8]$} & {$[1560.3,1565.6]$} \\
\hline & GN7 & {$[93.9,94.9]$} & {$[159.8,160.9]$} & {$[2473.2,2490.4]$} & {$[55.0,56.7]$} & {$[103.8,105.1]$} & {$[586.1,588.4]$} \\
\hline \multirow[t]{3}{*}{ LBG-GBDH } & $\mathrm{RPi}$ & {$[246.1,247.7]$} & {$[438.8,441.3]$} & {$[6335.5,6357.1]$} & {$[107.7,108.1]$} & {$[247.9,248.5]$} & {$[1597.3,1598.9]$} \\
\hline & MM1 & {$[236.8,239.1]$} & {$[419.4,425.2]$} & {$[5928.2,5977.2]$} & {$[126.6,132.8]$} & {$[263.2,266.1]$} & {$[1559.2,1568.0]$} \\
\hline & GN7 & {$[93.3,94.2]$} & {$[158.7,159.8]$} & {$[2468.8,2480.4]$} & {$[54.6,56.6]$} & {$[102.4,103.0]$} & {$[583.3,585.8]$} \\
\hline \multirow[t]{3}{*}{ GOT-GBDH } & $\mathrm{RPi}$ & {$[199.6,201.3]$} & {$[359.7,362.0]$} & {$[5113.5,5134.6]$} & {$[67.5,67.9]$} & {$[151.1,151.6]$} & {$[958.3,959.8]$} \\
\hline & MM1 & {$[194.6,196.2]$} & {$[345.4,351.5]$} & {$[4814.7,4866.4]$} & {$[81.2,83.3]$} & {$[162.7,165.2]$} & {$[972.0,1009.1]$} \\
\hline & GN7 & {$[77.2,78.2]$} & {$[131.7,132.9]$} & {$[1996.6,2006.3]$} & {$[38.2,40.4]$} & {$[65.7,66.5]$} & {$[362.6,364.4]$} \\
\hline \multirow[t]{3}{*}{ GNT2-GBDH } & $\mathrm{RPi}$ & {$[199.2,200.9]$} & {$[360.7,363.4]$} & {$[5120.2,5141.6]$} & {$[66.5,66.9]$} & {$[146.7,147.7]$} & {$[964.1,965.7]$} \\
\hline & MM1 & {$[195.5,198.1]$} & {$[345.4,348.8]$} & {$[4782.6,4822.6]$} & {$[81.6,83.1]$} & {$[160.8,163.3]$} & {$[977.4,1011.7]$} \\
\hline & GN7 & {$[77.4,78.2]$} & {$[132.4,133.7]$} & {$[1995.9,2006.7]$} & {$[37.5,39.5]$} & {$[66.0,67.2]$} & {$[361.3,363.8]$} \\
\hline
\end{tabular}

Tabela B.2: Intervalo de confiança (95\%) dos tempos de execução dos protocolos (em milissegundos), com pré-computação, para diferentes níveis de segurança. 


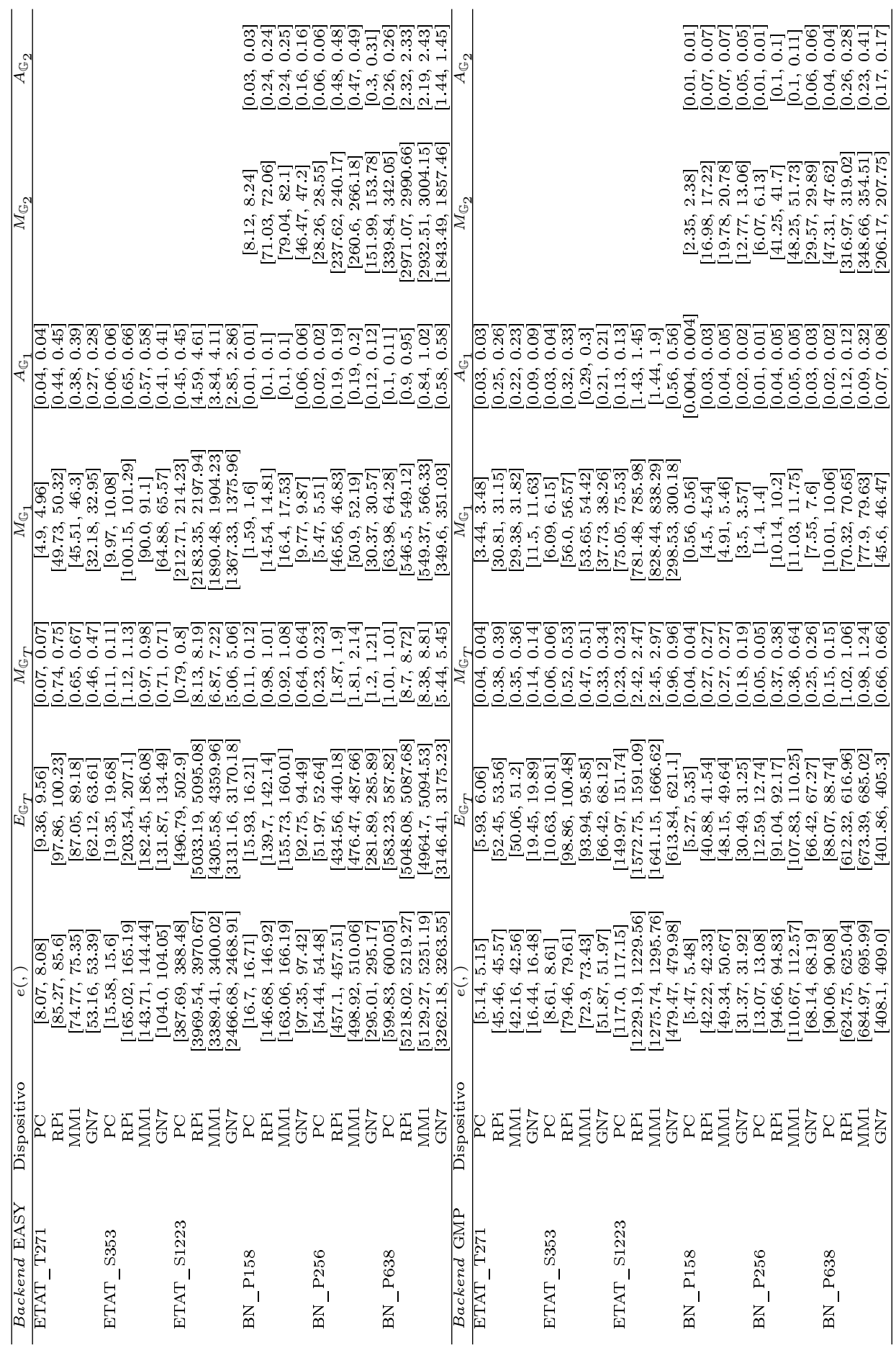

Tabela B.3: Intervalos de confiança (95\%) dos tempos de execução por operação (em milissegundos). 


\section{Referências Bibliográficas}

Al-Riyami e Paterson (2003) Sattam S Al-Riyami e Kenneth G Paterson. Certificateless public key cryptography. Em Advances in Cryptology - ASIACRYPT 2003, páginas 452-473. Springer. Citado na pág. 1, 14

Aranha e Gouvêa (2009) D. F. Aranha e C. P. L. Gouvêa. RELIC is an Efficient LIbrary for Cryptography. http://code.google.com/p/relic-toolkit/, 2009. Citado na pág. 36

Barker et al. (2007) E Barker, W Barker, WP Burr e M Smid. Nist sp800-57 recommendation for key management, part 1: General. Retrieved September, 5(2009):800-57. Citado na pág. xiii, 16

Barreto e Naehrig (2006) Paulo SLM Barreto e Michael Naehrig. Pairing-friendly elliptic curves of prime order. Em Selected areas in cryptography, páginas 319-331. Springer. Citado na pág. 37

Barreto et al. (2007) Paulo SLM Barreto, Steven D Galbraith, Colm O'Eigeartaigh e Michael Scott. Efficient pairing computation on supersingular abelian varieties. Designs, Codes and Cryptography, 42(3):239-271. Citado na pág. 38

Boneh e Franklin (2001) Dan Boneh e Matthew K. Franklin. Identity-based encryption from the weil pairing. Em Advances in Cryptology - CRYPTO 2001, páginas 213-229. Springer. Citado na pág. 14

Boneh e Franklin (2003) Dan Boneh e Matthew K. Franklin. Identity-based encryption from the Weil pairing. SIAM J. of Computing, 32(3):586-615. extended abstract in Crypto'01. Citado na pág. 11

Canetti e Krawczyk (2001) Ran Canetti e Hugo Krawczyk. Analysis of key-exchange protocols and their use for building secure channels. Em Advances in Cryptology-EUROCRYPT 2001, páginas 453-474. Springer. Citado na pág. 18

Cesena (2010) Emanuele Cesena. Trace Zero Varieties in Pairing-based Cryptography. Tese de Doutorado, Facoltà di Scienze Matematiche, Università degli Studi Roma Tre. Citado na pág. xi, 10

Chow e Choo (2007) Sherman SM Chow e Kim-Kwang Raymond Choo. Strongly-secure identity-based key agreement and anonymous extension. Em Information Security, páginas 203-220. Springer. Citado na pág. 24,42

Cocks (2001) Clifford Cocks. An identity based encryption scheme based on quadratic residues. Em IN IMA INT. CONF, páginas 360-363. Springer-Verlag. Citado na pág. 14

Cormen et al. (2009) Thomas H. Cormen, Charles E. Leiserson, Ronald L. Rivest e Clifford Stein. Introduction to Algorithms, Third Edition. The MIT Press, 3rd ed. ISBN 0262033844, 9780262033848. Citado na pág. 10

Dent (2010) Alexander W. Dent. Choosing key sizes for cryptography. Inf. Secur. Tech. Rep., 15(1):21-27. ISSN 1363-4127. Citado na pág. 16

Diffie e Hellman (1976) W. Diffie e M. E. Hellman. New directions in cryptography. IEEE Transactions on Information Theory, IT-22:644-654. Citado na pág. 1, 4, 11, 16

Freeman et al. (2010) David Freeman, Michael Scott e Edlyn Teske. A taxonomy of pairing-friendly elliptic curves. Journal of Cryptology, 23(2):224-280. Citado na pág. 37

Galbraith et al. (2006) Steven D. Galbraith, Kenneth G. Paterson e Nigel P. Smart. Pairings for cryptographers. IACR Cryptology ePrint Archive, 2006:165. Citado na pág. 10

Goya et al. (2010) Denise Goya, Cleber Okida e Routo Terada. A two-party certificateless authenticated key agreement protocol. SBSeg 2010, X Simpósio Brasileiro de Segurança da Informação e de Sistemas Computacionais, páginas 443-446. Citado na pág. 29, 31, 42

Goya et al. (2011) Denise Goya, Dionathan Nakamura e Routo Terada. Acordo de chave seguro contra autoridade mal intencionada. SBSeg 2011, XI Simpósio Brasileiro de Segurança da Informação e de Sistemas Computacionais, páginas 265-278. Citado na pág. 32, 42 
Goya (2006) Denise Hideko Goya. Proposta de esquemas de criptografia e de assinatura sob modelo de criptografia de chave pública sem certificado. Dissertação de Mestrado, Instituto de Matemática e Estatística da Universidade de São Paulo. Citado na pág. 6

Goya (2011) Denise Hideko Goya. Criptografia de chave pública sem certificado. Tese de Doutorado, Instituto de Matemática e Estatística da Universidade de São Paulo. Citado na pág. xi, 12, 16, 18, 19, 28, 31, 33, 42

Hankerson et al. (2003) Darrel Hankerson, Alfred J. Menezes e Scott Vanstone. Guide to Elliptic Curve Cryptography. Springer-Verlag New York, Inc., Secaucus, NJ, USA. ISBN 038795273X. Citado na pág. 56, 57

Hoffstein et al. (2008) Jeffrey Hoffstein, Jill Pipher e J.H. Silverman. An Introduction to Mathematical Cryptography. Springer Publishing Company, Incorporated, 1 ed. ISBN 0387779930, 9780387779935. Citado na pág. 55, 56

Hu et al. (2009) Xuexian Hu, Wenfen Liu e Jianhui Zhang. An efficient id-based authenticated key exchange protocol. Em Information Engineering, 2009. ICIE'09. WASE International Conference on, volume 2, páginas 229-233. IEEE. Citado na pág. 23, 42

Huang e Cao (2009) Hai Huang e Zhenfu Cao. An id-based authenticated key exchange protocol based on bilinear diffie-hellman problem. Em Proceedings of the 4th International Symposium on Information, Computer, and Communications Security, páginas 333-342. ACM. Citado na pág. 23, 40, 42

Júnior (2003) Waldir Dias Benits Júnior. Sistemas criptográficos baseados em identidades pessoais. Dissertação de Mestrado, Instituto de Matemática e Estatística da Universidade de São Paulo. Citado na pág. 5, 8

Koblitz (1987) Neal Koblitz. Elliptic curve cryptosystems. Mathematics of computation, 48(177):203-209. Citado na pág. 57

Krawczyk (2005) Hugo Krawczyk. Hmqv: A high-performance secure diffie-hellman protocol. Em Advances in Cryptology-CRYPTO 2005, páginas 546-566. Springer. Citado na pág. 17, 18

LaMacchia et al. (2007) Brian LaMacchia, Kristin Lauter e Anton Mityagin. Stronger security of authenticated key exchange. Em Provable Security, páginas 1-16. Springer. Citado na pág. 17, 18

Lippold e Nieto (2010) Georg Lippold e Juan González Nieto. Certificateless key agreement in the standard model. Em Proceedings of the Eighth Australasian Conference on Information Security-Volume 105, páginas 7585. Australian Computer Society, Inc. Citado na pág. 19

Lippold et al. (2009) Georg Lippold, Colin Boyd e Juan Gonzalez Nieto. Strongly secure certificateless key agreement. Em Proceedings of the 3rd International Conference Palo Alto on Pairing-Based Cryptography, Pairing '09, páginas 206-230, Berlin, Heidelberg. Springer-Verlag. ISBN 978-3-642-03297-4. Citado na pág. 19, 28, 29,42

Menezes et al. (1996) Alfred J. Menezes, Scott A. Vanstone e Paul C. Van Oorschot. Handbook of Applied Cryptography. CRC Press, Inc., Boca Raton, FL, USA, 1st ed. ISBN 0849385237. Citado na pág. 4, 5, 17

Miller (1986) Victor S Miller. Use of elliptic curves in cryptography. Em Lecture notes in computer sciences; 218 on Advances in cryptology-CRYPTO 85, páginas 417-426, New York, NY, USA. Springer-Verlag New York, Inc. ISBN 0-387-16463-4. URL http://dl.acm.org/citation.cfm?id=18262.25413. Citado na pág. 57

Ni et al. (2011) Liang Ni, Gongliang Chen, Jianhua Li e Yanyan Hao. Strongly secure identity-based authenticated key agreement protocols. Computers \& Electrical Engineering, 37(2):205-217. Citado na pág. 25, 26, 42

Ni et al. (2012) Liang Ni, Gongliang Chen e Jianhua Li. Escrowable identity-based authenticated key agreement protocol with strong security. Computers $\& 3$ Mathematics with Applications. Citado na pág. 26, 42

Okamoto e Pointcheval (2001) Tatsuaki Okamoto e David Pointcheval. The gap-problems: A new class of problems for the security of cryptographic schemes. Em Proceedings of PKC 2001, volume 1992 of LNCS, páginas 104-118. Springer-Verlag. Citado na pág. 11

Rivest et al. (1978) R.L. Rivest, A. Shamir e L. Adleman. A method for obtaining digital signatures and public-key cryptosystems. Communications of the ACM, 21:120-126. Citado na pág. 4

Shamir (1984) Adi Shamir. Identity-based cryptosystems and signature schemes. Em Proceedings of CRYPTO 84 on Advances in cryptology, volume LNCS 196, páginas 47-53. Springer-Verlag New York, Inc. ISBN 0-387-15658-5. Citado na pág. $1,13,14$

Swanson e Jao (2009a) Colleen Swanson e David Jao. A study of two-party certificateless authenticated keyagreement protocols. Em INDOCRYPT '09: Proceedings of the 10th International Conference on Cryptology in India, páginas 57-71, Berlin, Heidelberg. Springer-Verlag. ISBN 978-3-642-10627-9. Citado na pág. 16 
Swanson e Jao (2009b) Colleen Swanson e David Jao. A study of two-party certificateless authenticated keyagreement protocols. Em Progress in Cryptology-INDOCRYPT 2009, páginas 57-71. Springer. Citado na pág. 18, 19

Swanson (2008) Colleen Marie Swanson. Security in key agreement: Two-party certificateless schemes. Dissertação de Mestrado, University of Waterloo. Citado na pág. 18

Terada (2008) Routo Terada. Segurança de Dados. Blucher, 2 ed. ISBN 9788521204398. Citado na pág. 5, 6, 7, 11, 16,55

Vercauteren (2010) Frederik Vercauteren. Optimal pairings. Information Theory, IEEE Transactions on, 56(1): 455-461. Citado na pág. 38 